\title{
Floods of May-July 1950 in Southeastern Nebraska
}

Prepared by WATER RESOURCES DIVISION

FLOODS OF 1950

GEOLOGICAL SURVEY WATER-SUPPLY PAPER 1137-D

A presentation of data on four floods: Little Nemaha River basin and Salt Creek on May 8-9, Union and Shell Creeks on June 2, West Fork Big Blue River and Beaver Creek on July 8-9, and Shell and Plum

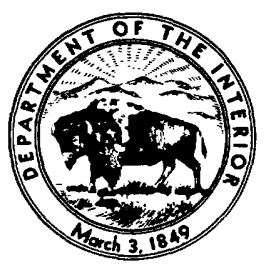
Creeks on July 18. Prepared in cooperation with the Nebraska Department of Roads and Irrigation. 
UNITED STATES DEPARTMENT OF THE INTERIOR

Douglas McKay, Secretary

\section{GEOLOGIGAL SURVEY}

W. E. Wrather, Director 


\section{CONTENTS}

Abstract $\ldots \ldots \ldots \ldots \ldots$

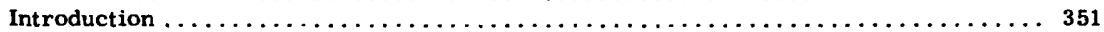

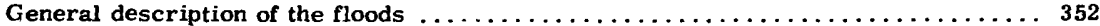

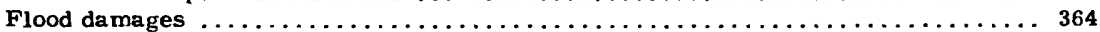

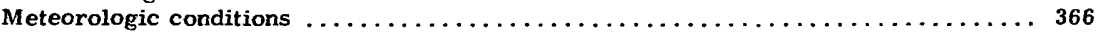

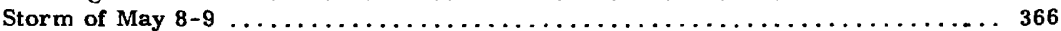

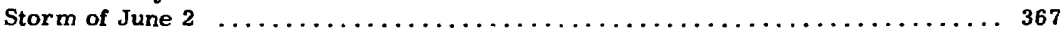

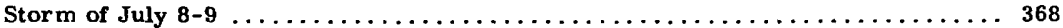

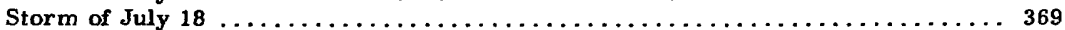

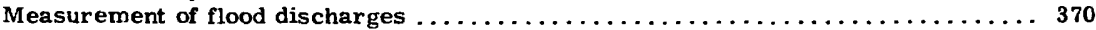

Stages and discharges at stream-gaging stations $\ldots \ldots \ldots \ldots \ldots \ldots \ldots \ldots \ldots \ldots \ldots$

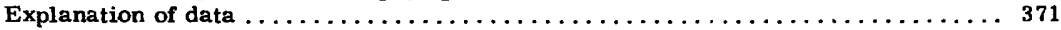

Missouri River main stem ...................................... 373

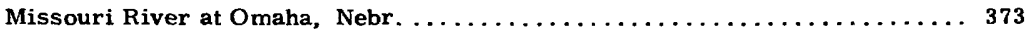

Missouri River at Nebraska City, Nebr. . . . . . . . . . . . . . . 373

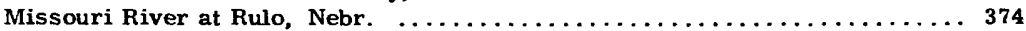

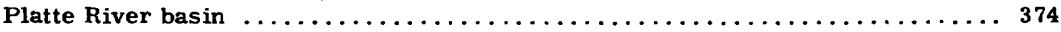

Platte River near Grand Island, Nebr. $\ldots \ldots \ldots \ldots \ldots \ldots \ldots \ldots \ldots \ldots \ldots \ldots \ldots$

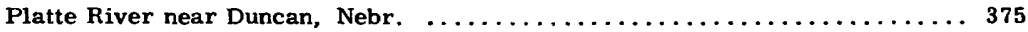

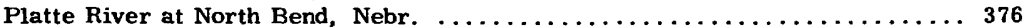

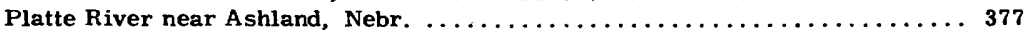

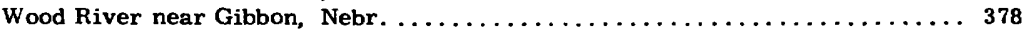

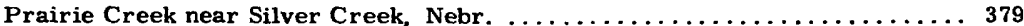

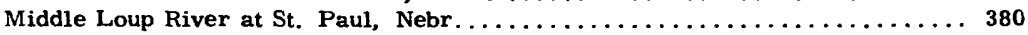

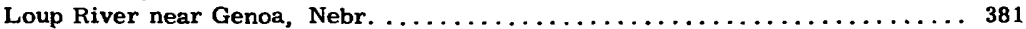

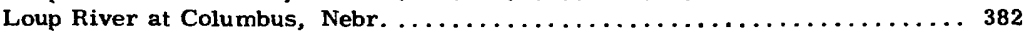

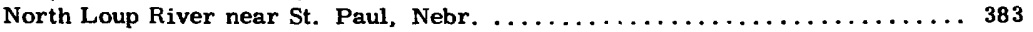

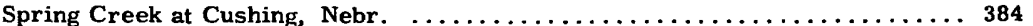

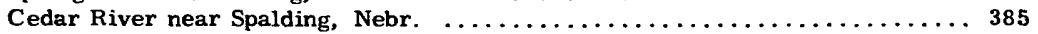

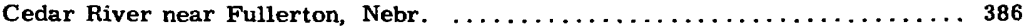

Loup River power canal near Genoa, Nebr. ................. 387

Beaver Creek at Loretto, Nebr. $\ldots \ldots \ldots \ldots \ldots \ldots \ldots \ldots \ldots \ldots \ldots \ldots \ldots \ldots$

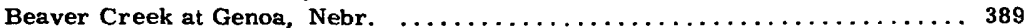

Shell Creek at Newman Grove, Nebr. $\ldots \ldots \ldots \ldots \ldots \ldots \ldots \ldots \ldots \ldots \ldots \ldots \ldots . \ldots \ldots$

Shell Creek near Columbus, Nebr. . . . . . . . . . . . . . . . . . . . 391

Elkhorn River at Norfolk, Nebr. . . . . . . . . . . . . . . . . . . 392

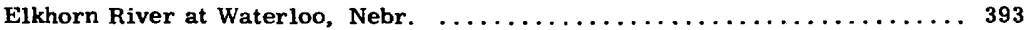

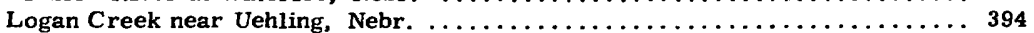

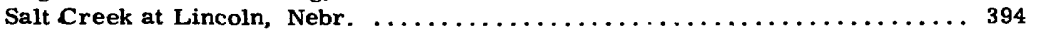

Salt Creek near Ashland, Nebr. . . . . . . . . . . . . . . . . . . . . 395

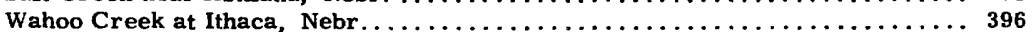

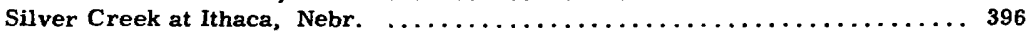

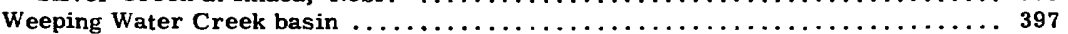

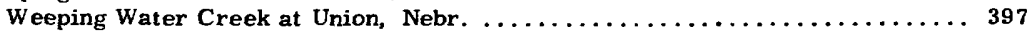

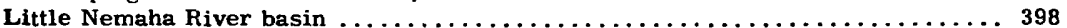

Little Nemaha River at Auburn, Nebr. ... . . . . . . . . . . . . . 398

Nemaha River basin .................................. 399

Nemaha River at Falls City, Nebr. ......................... 399

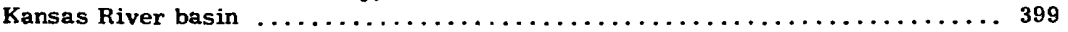

Republican River near Bloomington, Nebr. . . . . . . . . . . . . . . 399

Republican River near Hardy, Nebr.....................400

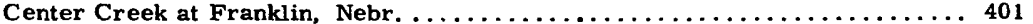

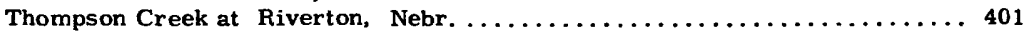

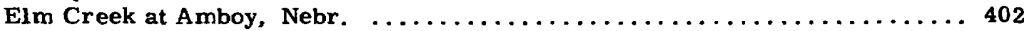

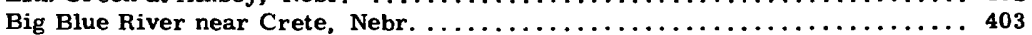

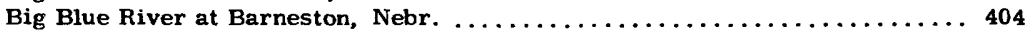

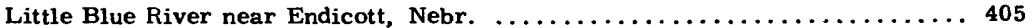

Summary of flood stages and discharges $\ldots \ldots \ldots \ldots \ldots \ldots \ldots \ldots \ldots \ldots \ldots \ldots \ldots \ldots \ldots \ldots 406$ 
Figure 50. Map of Nebraska showing area covered by this report and places where peak discharges were determined $\ldots \ldots \ldots \ldots \ldots \ldots \ldots \ldots$

51. Hydrographs of Little Nemaha and Loup Rivers, showing flood activity during May-July $1950 \ldots \ldots \ldots \ldots \ldots \ldots \ldots \ldots \ldots \ldots \ldots \ldots \ldots$

52. Isohyetal map of southeastern Nebraska and western Iowa showing

total precipitation, in inches, May $8-9,1950 \ldots \ldots \ldots \ldots \ldots \ldots \ldots \ldots$
53. View along $U$. S. Highway 40 east of Unadilla, indicating height reached

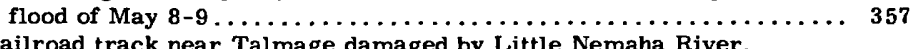

54. Railroad track near Talmage damaged by Little Nemaha River,

May $8-9 \ldots \ldots \ldots \ldots \ldots \ldots \ldots \ldots \ldots \ldots \ldots \ldots \ldots \ldots \ldots \ldots \ldots \ldots \ldots \ldots$
5. S. Highway $73-75$ north of Auburn inundated by Little Nemaha

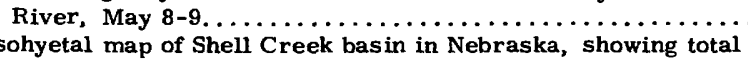
56. Isohyetal map of Shell Creek basin in Nebraska, showing total
precipitation, in inches, June $1-2,1950 \ldots \ldots \ldots \ldots \ldots \ldots \ldots \ldots \ldots$

57. Isohyetal map of southeastern Nebraska and northeastern Kansas, showing total precipitation, in inches, July $8-9,1950 \ldots \ldots \ldots \ldots . \ldots 1$

58. Graph showing cumulative precipitation, in inches, at York, Nebr.,

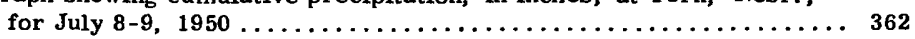

59. Beaver Crossing flooded by Beaver Creek, July $9 \ldots \ldots \ldots \ldots \ldots \ldots \ldots$

60. State Highway 39 near Genoa damaged by Beaver Creek, July $19 \ldots \ldots 3$

61. Relation of unit discharge to size of drainage area. . . . . . . . 407

\section{TABLES}

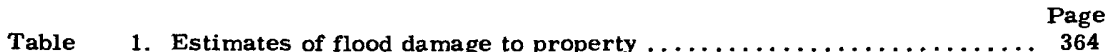

2. Agricultural and land damage caused by flood of May $8-9,1950 \ldots \ldots \ldots 36$

3. Summary of flood stages and discharges in southeastern Nebraska, May-July 1950. 


\section{PREFACE}

This report on the floods of May-July 1950 in southeast Nebraska was prepared by the Geological Survey, Water Resources Division, under the general direction of C. G. Paulsen, chief hydraulic engineer, and J. V. B. Wells, chief, Surface Water Branch.

The field work and the collection and tabulation of basic information relating to stage and discharge were done by the staff of the Lincoln district office of the Surface Water Branch under the supervision of Douglas D. Lewis, district engineer. Valuable assistance to the field and office work was given by R. E. Oltman and Hollister Johnson, hydraulic engineers.

The Nebraska Department of Roads and Irrigation, through the Bureau of Irrigation, Water Power, and Drainage, has cooperated financially with the Survey's regular streamflow measurement program in Nebraska, and the Corps of Engineers, U. S. Army, has given financial assistance in the maintenance of gaging stations within the area described in this report. The U. S. Weather Bureau, the Corps of Engineers, and the U. S. Soil Conservation Service supplied certain data for this report. 



\section{FLOODS OF 1950}

\section{FLOODS OF MAY-JULY 1950 IN SOUTHEASTERN}

NEBRASKA

Prepared by Water Resources Division 



\section{ABSTRACT}

Four floods occurred in southeast Nebraska during the period of May to July 1950. Two of these were the greatest known in the State if the size of the drainage areas is considered, and the other two were not so spectacular but were of notable size and of possible hydrologic significance in their relation to the $t w o$ major floods. Although property loss and damage have been exceeded in $\mathrm{p} r e-$ vious floods in Nebraska, notably in the flood of May-June 1935 on the Republican River, they were extremely high in the period covered by this report. Loss of life, which resulted largely from the rapid cresting of the streams, was likewise high.

Each of the floods was caused by heavy precipitation, which at times reached intensities seldom recorded in the Missouri River basin. On May 8, 1950, more than 14 inches of rain fell over certain areas of the Little Nemaha $R$ i ve $r$ basin within a period of a few hours. One center of the storm of July 8, 1950, occurred at York, Nebr., where 11 inches of rain was recorded within a 4-hour period, and the storm total exceeded 13 inches.

Notable high rates of discharge produced by the intense rainfall were 1,030 cfs per square $m i l e$ from 218 square miles of $d r a i n a g e$ area in the Little Nemaha River basin on May 9, 1950; 1, 020 cfs per square mile from 2.5 square miles drainage area in the Elkhorn River basin on June 2, 1950; and 3,320 cfs per square mile from $6.93 \mathrm{square}$ miles of $\mathrm{dra}$ inage area in the Big Blue River basin on July 9, 1950.

This report presents records of stage and discharge for the flood periods at 36 stream-gaging stations in southeast Nebraska, and a summary of peak discharges, with comparative data for previously known floods at 45 measurement points. The report also includes a discussion of the weather associated with the floods and other data pertinent to the floods.

\section{INTRODUCTION}

Heavy precipitation over the southeastern corner of Nebraska starting on the afternoon of May 8, 1950, c a u sed the first of a series of floods that extended through June and July. The flood of May 8-9 caused the loss of 23 lives, it inundated more than 60, 000 acres of land, and when estimates of damages to land and agriculture are included, it caused property damage in excess of 60 million dollars. This first storm was centered ove $r$ the head waters of Little Nemaha River and Salt Creek, but heavy precipitation a ls o caused floods of varying degrees of intensity along 
Weeping Water Creek, Nemaha River, and tributaries of the Big Blue River.

On June 2, 1950, heavy rainfall in Madison, Boone, and Platte Counties in Nebraska caused severe flooding of Union and Shell Creeks which inundated sections of New man Grove and Madison.

On July 8-9, 1950, further intense precipitation centered over York, Nebr., and caused unprecedented flooding of the West Fork Big Blue River and Beaver Creek. This storm also extended over the Loup River basin and caused flooding of some of the minor tributaries.

On July 18, other heavy rains fell in the Loup River basin. Beaver Creek at the Loretto and Genoa gaging stations and Cedar River near Spalding had record high stages and discharges.

The United States Geological Survey, through the district office of its Surface Water B ranch in Lincoln, Nebr., operates 36 gaging stations in the area affected by these floods, as a par of the regular Nation-wide stream gaging program for the investigation of the water resources of the Nation. These stations have been maintained by the Geological Survey largely in cooperation with the State and with other Federal agencies. Some of them have been operated continuously for periods of such length that systematic records of stage, discharge, and volume of flow covering the range from drought to extraordinary flood are available (fig. 50).

\section{GENERAL DESCRIPTION OF THE FLOODS}

The floods of May-July 1950 in southeas t Nebraska were the result of severe summer thunderstorms that reached cloudburst intensities in some localities. Figure 51 is a hydrograph of Little Nemaha and Loup Rivers, showing the discharges during the period May-July.

Late in the afternoon of May 8, heavy rain began falling over the southeastern part of Nebraska, and extremely heavy rain fell over an area that included the headwaters of Little Nemaha River, Salt Creek, and Weeping Water Creek, and over parts of the basins of the Big Blue River and Nemaha River (see fig. 52). The center of precipitation was a little east of the divide between Salt Creel and Little Nemaha River.

Salt Creek crested at the gaging station at Lincoln, Nebr., at 9 a. m. on May 9. It inundated large areas of the low-lying bridges, highways, and railways. Upstream from Lincoln agricultural lands were damaged considerably. Eleven thousand a c res and three towns wereflooded, and sixlives were lost in the Salt Creek flood. 


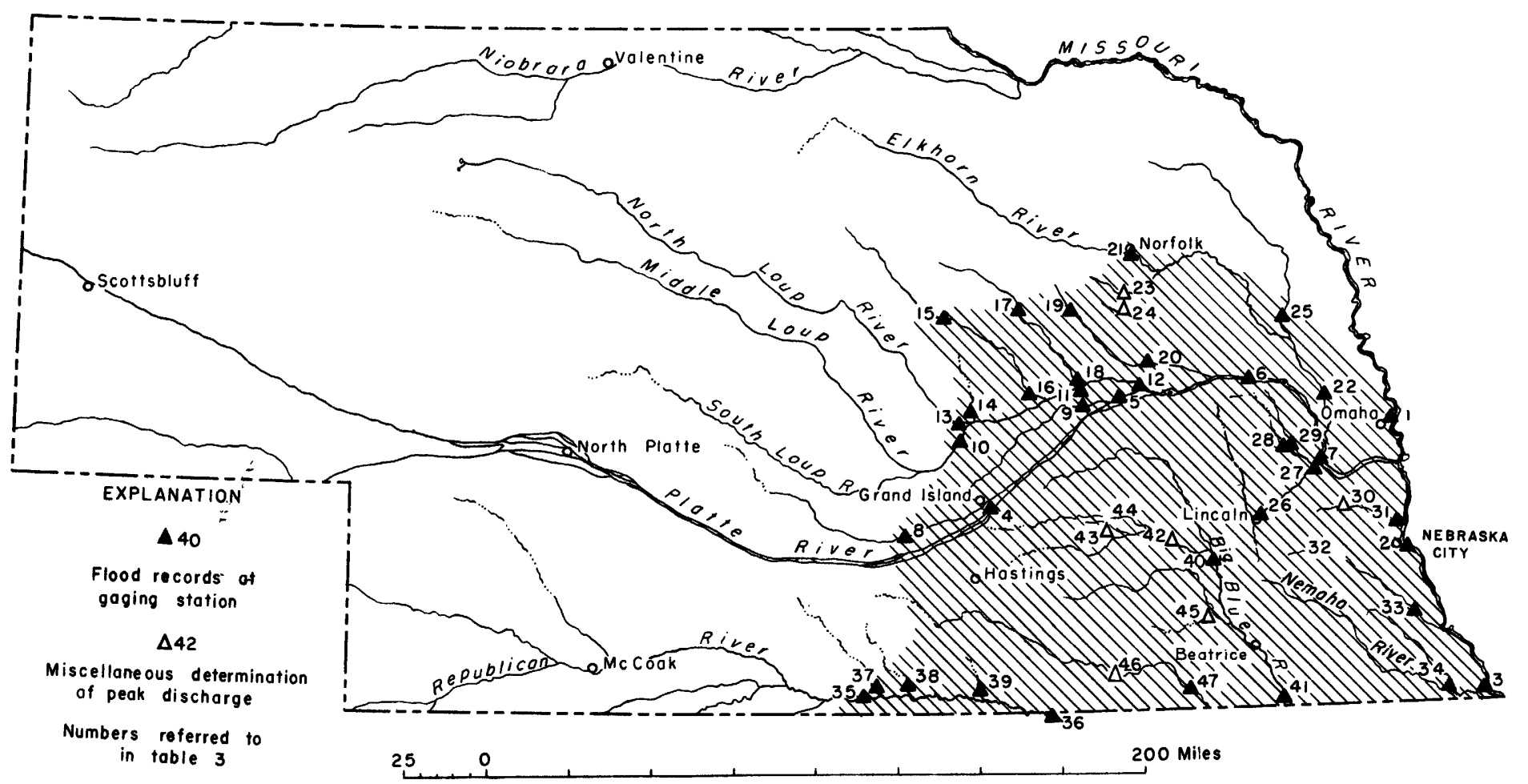

Figure 50.-Map of Nebraska showing area covered by this report and places where peak discharges were determined. 


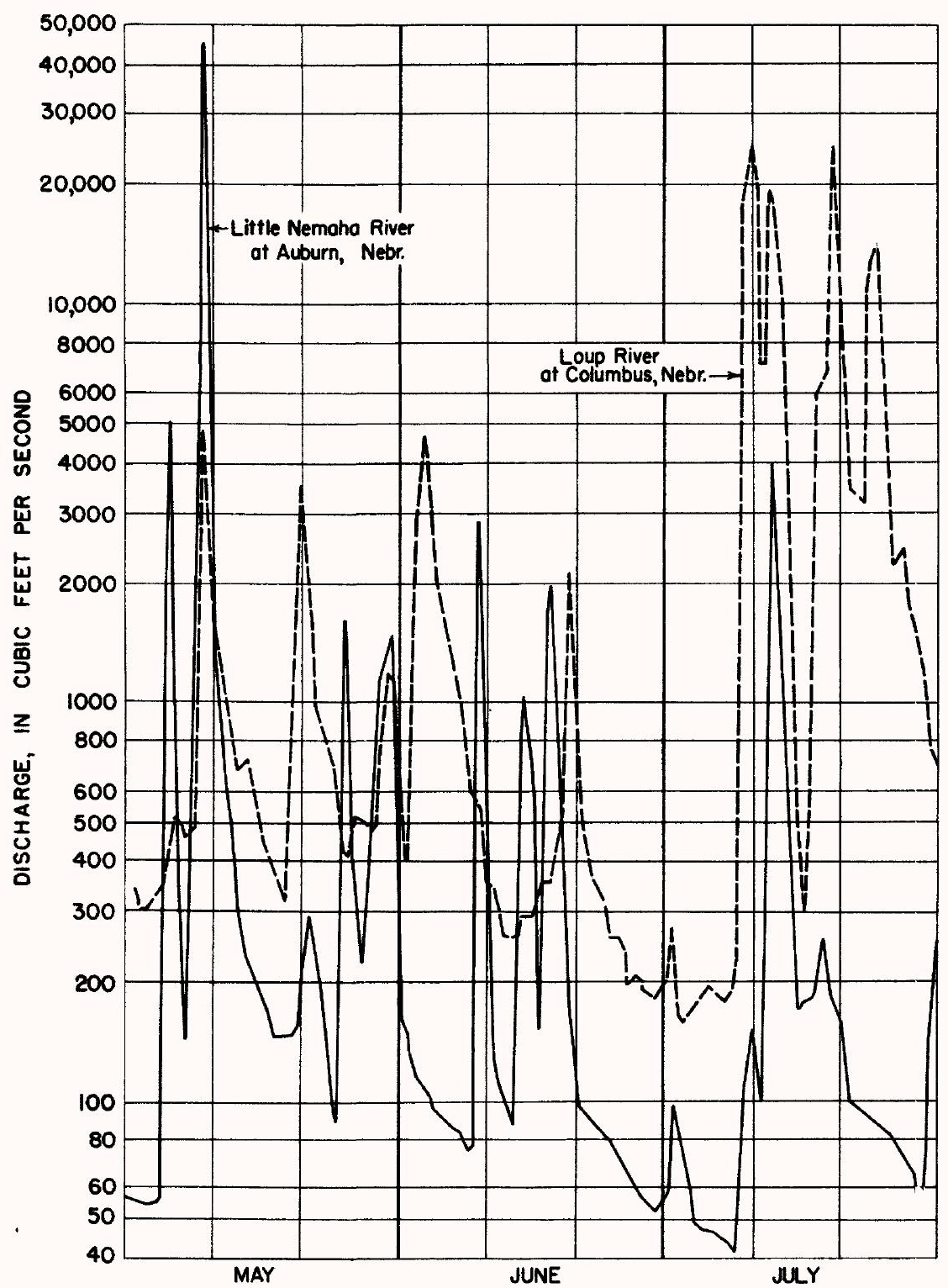

Figure 51.-Hydrographs of Little Nemaha and Loup Rivers, showing flood activity during May-July 1950. 


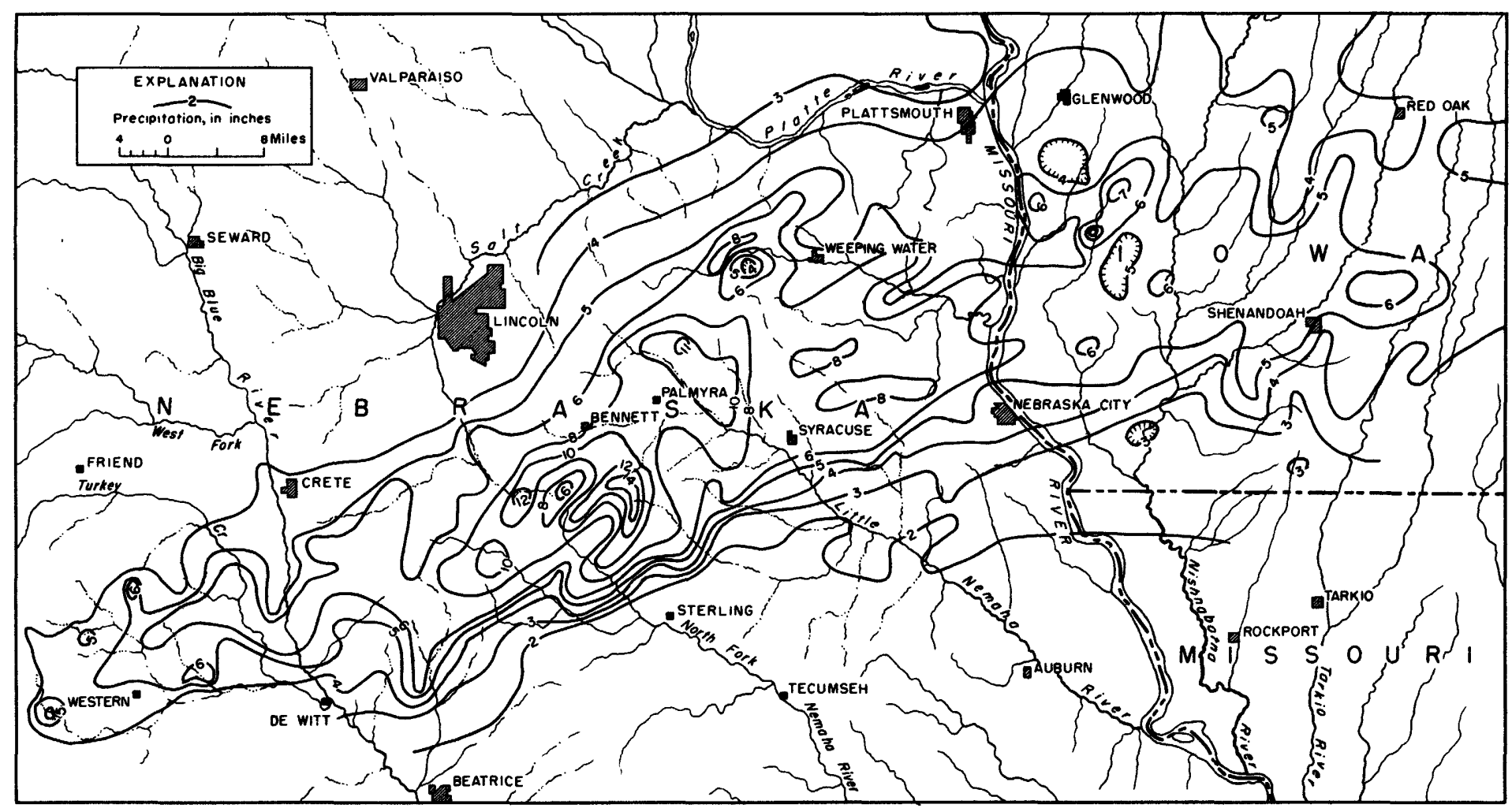

Figure 52.-Isohyetal map of southeastern Nebraska and western Iowa showing total precipitation, in inches, May 8-9, 1950. 
The crest discharge of Salt $\mathrm{Creek}$ at the Lincoln gaging station was $27,800 \mathrm{cfs}$. It is well established that the 1950 flood is the greates t since July 5, 1908. Because of many channel changes during the interim, largely man-made for flood protection, there is no direct means of comparing the 1908 and 1950 floods. On July 5, 1908, Adna Dobson, then Lincoln city engineer, made a current-meter measurement of 18,000 cfs "near the crest." Many years later a firm of consulting engineers computed the maximum discharge of the 1908 flood as $30,600 \mathrm{cfs}$. These computations were based on valley slopes and cross sections by the U. S. Department of Agriculture in 1909 and upon coefficients of roughness that are not subject to verification. It seems quite possible that the flood of May 9,1950, may have exceeded that of July 5, 1908, which would make it the largest since the valley was first inhabited.

It is worthy of note that Haines Branch, Middle Creek, and Oak Creek contributed very little to the peak discharge of Salt Creek on May 9, 1950, and that most of the runoff came from a relatively small area above Roca. Below Lincoln the crest discharge was reduced by valley storage, and at Ashland, near the mouth of Salt Creek, the flood was not unusual.

The most intense flows from the storm of May 8-9 developed in the Little Nemaha River basin. The river crested at Unadilla about midnight of May 8, covering the flood plain to depths of nearly $10 \mathrm{ft}$. (see fig. 53). Several farm homes and bridges were destroyed in this vicinity. About a mile east of Unadilla a bus was trapped by the rapidly rising waters. The 17, 500-pound vehicle was swept from the highway and carried two miles to be deposited in the river channel near Syracuse. The lives of 7 persons were claimed by this misfortune. Between Unadilla and Syracuse, the Chicago, Burlington \& Quincy railroad line was t or n up, bridges were washed out, and sheds and machinery wereswept away from low-lying farms. Figure 54 shows da mage to tracks of the Missouri Pacific Railroad near Talmage.

The flood crest of the Little Nemaha River reached Syracuse about 1 a. m. May 9 . In the low areas of town, residences were moved from their foundations and broken against other buildings and trees. The flood plain here is more than a mile wide, ant after the flood it was littered with stock tanks, grain bins, windmills, household furnishings, fragments of buildings, bridges, and other destroyed property. A computation of discharge by contracted-opening and flow-over-embankment methods indicate a discharge at Syracuse of 225,000 cfs $f \mathrm{r}$ m a drainage area $\mathrm{c}^{f}$ 218 square miles.

The flood crest reached Auburn at about 7 a.m. May 9. Between Syracuse and Auburn it swept over valuable farm lands ant inundated the village of Brock. Damage to bridges, highways 
(fig. 55), railroads, and farm property was particularly heavy.

In the Little Nemaha River basin, a total of 35,000 a cres and 8 towns were flooded, and 14 lives were lost including the 7 previously mentioned as lost near Unadilla.

Weeping Water Creek crested early on May 9. The villages of Weeping Water and Nehawka were badly flooded, highways and rail roads were da maged, and crops on the lowlands were destroyed. At Union, several residences were completely destroyed, the yards of the Missouri Pacific Railroad were damaged and severa 1 trestles washed out. One life was lost at Union. The crest discharge at Weeping Water was $30,300 \mathrm{cfs}$, and at Union it was $60,300 \mathrm{cfs}$, which is more than twice as large as the highest previously known flood of June 1947.

In the Big Blue River basin principal damage occurred when Turkey Creek inundated the village of DeWitt. Every house i n the community of 500 people was flooded, the only dry area being a spot on the main street big enough to park the fire truck. Th e crest discharge of Turkey Creek on May 9 was determined by the slope-area method to be 21, $400 \mathrm{cfs}$. Flooding was not severe on the main stem of the Big Blue River. About 8 miles of railroad track was washed out on the Lincoln-Beatrice line of the Union Pacific, and the basements of 17 homes in Beatrice were flooded. Two lives were lost when an automobile was swept from a highway between Beatrice and Wilber.

The Nemaha River bas in was largely outside the area of intense precipitation and flooding was not great. A crest discharge of 26, $300 \mathrm{cfs}$ oc curred at Falls City at 10:30 p. m., M a y 9. A total of 20,000 acres was flooded, 5 towns were m o d e r a tely flooded, but no lives were lost in the basin.

Early on the morning of June 2, thunderstorms broke out in Madison, Boone, and Platte Counties. Heaviest precipitation was centered on the headwaters of Shell Creek and another center only slightly less intense occurred on the headwaters of Union Creek. Figure 56 is an isohyetal map of Shell Creek basin, showing total precipitation, in inches, June 1-2, 1950. Flash floods developed quickly on these two streams and resulted in considerable damage to agricultural lands and small towns.

Shell Creek at Newman Grove reached a crest discharge of $11,600 \mathrm{cfs}$ at 5:30 a. m., June 2. By the time the crest reached the gaging station near Columbus at 12:30 p. m., June 3, valley storage had reduced the peak discharge to $5,970 \mathrm{cfs}$. 
Union Creek at Madison inundated much of the town on June 2 and had a peak discharge of $15,700 \mathrm{cfs}$ from a drainage area of 167 square miles. A normally dry tributary to Union Creek just south of Madison had a discharge of 2,560 cfs from 2.5 square miles.

Heavy runoff also developed on Beaver Creek. A peak discharge of $4,570^{\circ} \mathrm{cfs}$ occurred at $11 \mathrm{a} . \mathrm{m}$., June 2, at the Loret to gaging station. It is greatly in excess of any previous discharges observed during the 6 years of record at this station. Thecrest arrived at the Genoa gaging station at $10 \mathrm{p} . \mathrm{m}$. the same day. The peak discharge at this point was $8,470 \mathrm{cfs}$, or more than twice as great as the maximum previously recorded in 10 years of station operation.

On the afternoon of July 8, scattered showers and thunderstorms developed in eastern Nebraska. By midnight torrential rains had fallen, with the greatest amounts centered in the area near York on the headwaters of West Fork Big Blue River. Figure 57 is an isohyetal map of southeastern Nebraska and northeastern Kansas, and.figure 58 is a graph showing cumulative precipitation at Yorkfor the storm of July 8-9. Another storm center associated with the same disturbance was south and west near Jewell, Kans.; it resulted in heavy precipitation falling on certain tributaries to the Republican River between Bloomington and Hardy.

The most intense flood flows developed on tributaries to West Fork Big Blue River in the vicinity of York (fig. 57). Residential and business sections of York were badly flooded by Beaver Creek, and rural homes in the low-lying areas were damaged. At Beaver Crossing almost the entire business district and a large part of the residential area were inundated (fig. 59). An unnamed tributary to West Fork Big Blue River about 6 miles south of York produced 23, $000 \mathrm{cfs}$ from a drainage area of 6.93 square miles, computed by the slope-area method. Another tributary a little farther south produced $15,200 \mathrm{cfs}$ from an area of 17.2 square miles.

The West Fork Big Blue River crested at Dorchester on July 10 at a peak discharge of $49,400 \mathrm{cfs}$. At $\mathrm{Crete}$ on the main stem of the Big Blue River the peak discharge was reduced by valley storage to $27,600 \mathrm{cfs}$, the crest occurring at $1 \mathrm{p}$. m., July 10 . The lower parts of $\mathrm{Crete}$ wereflooded and serious damage extended downstream below Beatrice. At the Barneston gaging station the flow was confined to the banks of the stream. The floods at York, Beaver Crossing, and Crete were greater than any others known there. At Beatrice and points downstream greater floods have been known in the past. 


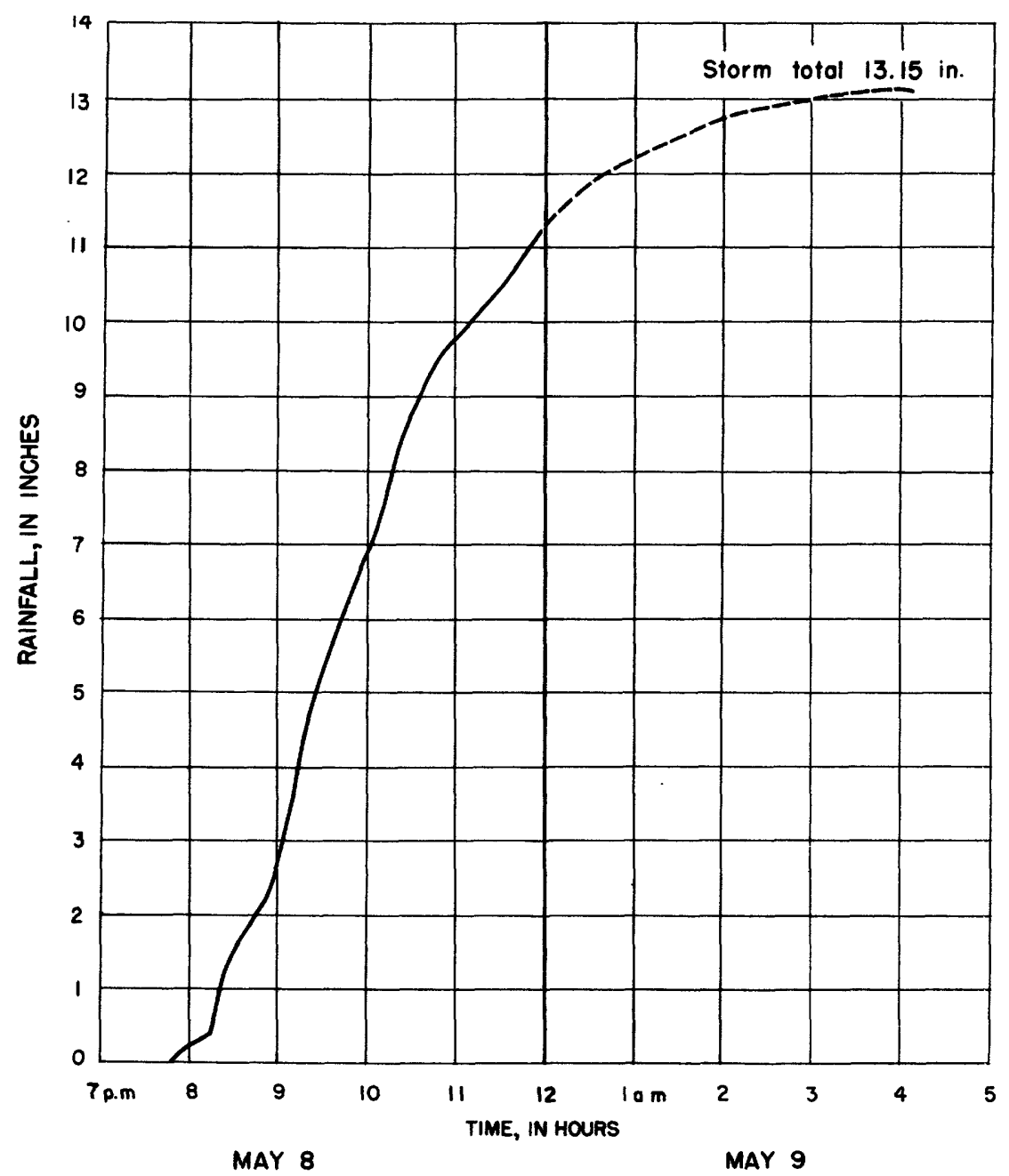

Frgure 58.-Graph showing cumulative precipitation, in inches, at York, Nebr.. for July 8-9, 1950.

at the Fullerton gaging station reached a crest discharge of $10,100 \mathrm{cfs}$ at $1: 30$ a.m., July 19, thereby exceeding the previous maximum of $9,760 \mathrm{cfs}$ for the period of record covering 1931-32, 1940-50. Beaver Creek, having already set two new record discharges during these summer floads at the Genoa gaging station, reached a crest discharge of $21,200 \mathrm{cfs}$ at 2:30 a. m., July 19, which was far in excess of the previous record-breaking 9,100 cfs established only 10 days earlier. Figure 60 shows damage to State Highway 39 near Genoa. 


\section{FLOOD DAMAGES}

At least 24 lives were lost as a result of the floods of MayJuly 1950. Twenty-three known deaths resulted from the flood of May 8-9, and one was caused by the flood of July 8-10. Much of this loss of life occurred when automobiles were trapped by the rapidly rising waters and swept from the highways.

Immediately after the floods, surveys of flood damage were begun by the Corps of Engineers and the Soil Conservation Service. The intensity of field work done in these surveys was proportional to the magnitude of individual storms.

The tabulation of flood damage by basins, shown in table 1 , was abstracted from material furnished by the Missouri River Division, Corps of Engineers.

Table 1. --Estimates of flood damage to property

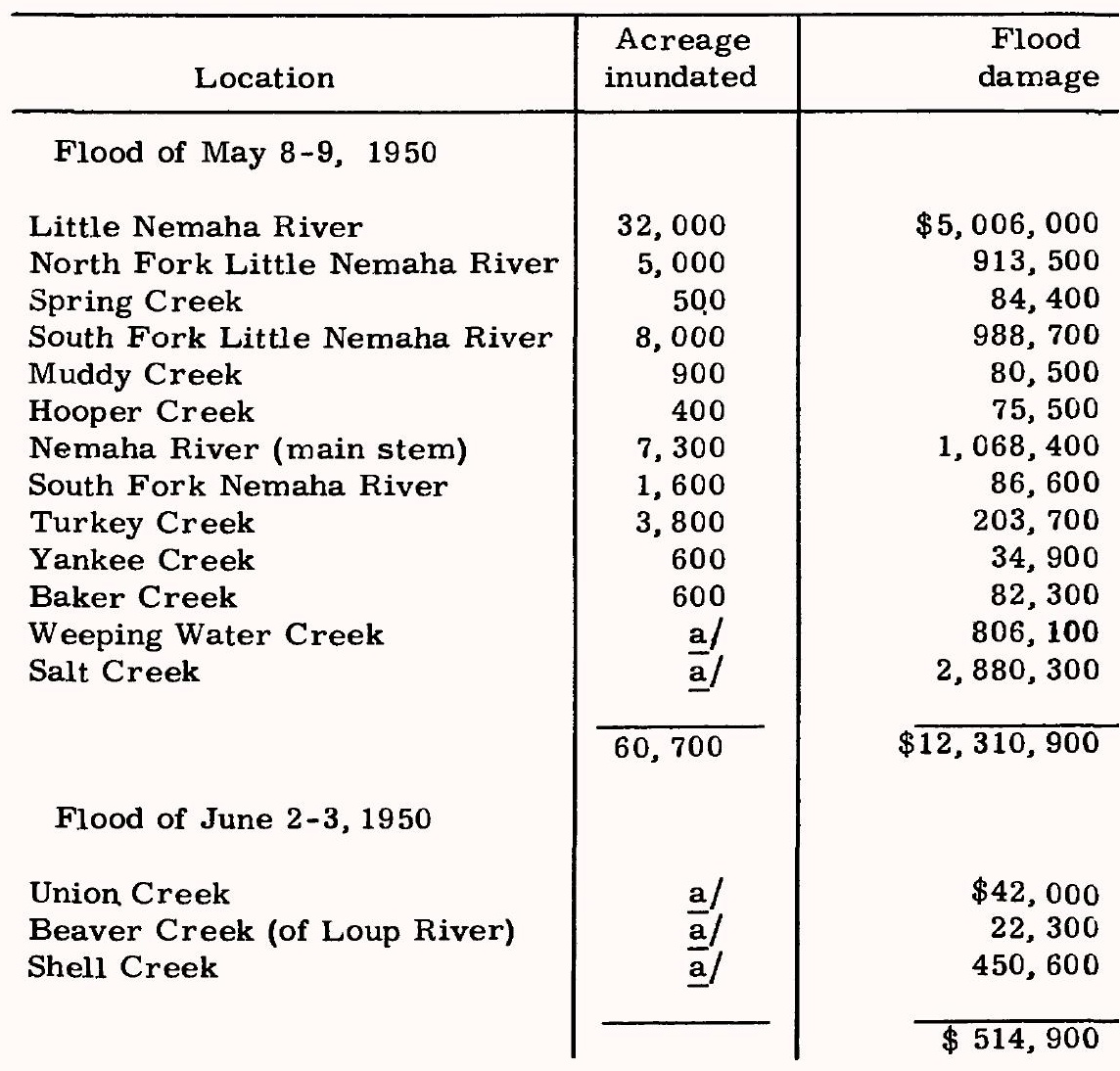


Table 1. --Estimates of flood damage to property--Continued.

\begin{tabular}{|c|c|c|}
\hline Location & \begin{tabular}{c|} 
Acreage \\
inundated
\end{tabular} & $\begin{array}{l}\text { Flood } \\
\text { damage }\end{array}$ \\
\hline \multicolumn{3}{|l|}{ Flood of July $8-10,1950$} \\
\hline Big Blue River & 30,100 & $\$ 468,400$ \\
\hline Lincoln Creek & 4,300 & 21,300 \\
\hline $\begin{array}{l}\text { West Fork Big Blue River (main } \\
\text { stem) }\end{array}$ & 16,300 & 734,200 \\
\hline $\begin{array}{l}\text { West Fork Big Blue River } \\
\text { (Beaver Creek) }\end{array}$ & 5,300 & 484,200 \\
\hline Big Indian Creek & 3,800 & 39,200 \\
\hline Little Blue River & 8,900 & 79,400 \\
\hline Rose Creek & 3,300 & 62,700 \\
\hline & 72,000 & $\$ \overline{1,889,400}$ \\
\hline \multicolumn{3}{|l|}{ Flood of July 18, 1950} \\
\hline Shell Creek & a/ & 68,800 \\
\hline Plum Creek & $\underline{\underline{a}} /$ & 304,800 \\
\hline Grand total & 132,700 & $\$ 15,088,800$ \\
\hline
\end{tabular}

a/ Not available as this was written.

Agricultural and land damages cau s ed by the flood of May 8-9 presented in table 2 were extracted from the Department of Agriculture, Soil Conservation Service Report "Floodwater and sediment damages in southeast Nebraska resulting from storm of May 8-9, 1950." In its report the Soil Conservation Service defines agricultural damage as damage to crops and pasture, stored grain and hay, farm improvements, livestock, and general cleanup costs; and land damage as erosion, harmful deposition of sediments, and swamping on bottomlands.

The tabulations for agriculturaldamage may duplicate to some extent certain items included in table 1. Similar information relating to agricultural and land damage is not available for subsequent floods in June and July. 
Table 2. --Agricultural and land damage caused by flood of May 8-9, 1950

\begin{tabular}{|c|c|c|c|}
\hline Location & $\begin{array}{c}\text { Agricultural } \\
\text { damage }\end{array}$ & $\begin{array}{c}\text { Land } \\
\text { damage }\end{array}$ & Total \\
\hline Salt Creek & $\$ 230,384$ & $\$ 9,028,561$ & $\$ 9,258,945$ \\
\hline Little Nemaha River & 944,306 & $12,218,679$ & $13,162,985$ \\
\hline Nemaha River & 189,033 & $3,094,011$ & $3,283,044$ \\
\hline Weeping Water Creek & 120,156 & $6,113,548$ & $6,233,704$ \\
\hline Big Blue River & 175,639 & $14,227,078$ & $14,402,717$ \\
\hline Platte River tributaries & 7,749 & $1,891,963$ & $1,899,712$ \\
\hline Missouri River tribu- & 5,171 & $1,575,644$ & $1,580,815$ \\
\hline Total & $\overline{\$ 1,672,438}$ & $\$ 48,149,484$ & $\overline{\$ 49,821,922}$ \\
\hline
\end{tabular}

\section{METEOROLOGIC CONDITIONS}

Prepared by the staff of the United States Weather Bureau

Storm of May 8-9, 1950, in the basins of the Nemaha and Little Nemaha Rivers and Salt Creek

The rains over southeastern Nebraska on May 8, 1950, resulted in part from the lifting of warm moist air over polar air, and in part from instability showers in the cold air, intensified by the approach and passage of a developing cyclone.

Early on the afternoon of May 7 an intense low-pressure area on the eastern coast of Canada dominated the circulation westward to Hudson Bay. The polar front extended southwestward, from the low, skirting the New England coast, then across southern Pennsylvania to southern Kansas where it became a warm front that extended into another low in eastern Utah. A huge area of rain, snow, drizzle, and showers stretched northward from the warm front, covering parts of Montana, Wyoming, Colorado, South Dakota, western Nebraska, and the northwest corner of Kansas. The north-central part of the United States was under the influence of a well-developed polar high centered in Canada north-northeast of Minnesota. In the southeast, a nose of the warm Bermuda high projected over the States from Florida to Texas. By12: $30 \mathrm{a} . \mathrm{m}$. , local time, May 8, the warm front had advanced northward to the southern border of Nebraska. Surface winds north of the front were from the east; south of the front the winds were from the southwest. Aloft, the flow of air from the southwest over southern Nebraska indicated that the warm air was being lifted over the polar air. Showers fell over the Nemaha and Little Nemaha Rivers and Salt Creek in the early morning hours, but the amounts of rain 
were moderate. During the next 6 hours a wave formed in the northwest corner of Kansas. An inverted V-trough developed northward from the peak of the wave through the central part of the Great Plains. The precipitation area spread rapidly eastward with this development, and showers and thunderstorms broke out over most of eastern Nebraska.

The trough inched eastward and became more pronounced during the following 6 hours. The cold front of the wave moved slowly southeastward, and the precipitation over southeastern Nebraska, just ahead of the warm front, took the form of drizzle. In the evening, however, thunderstorms began again as the peak of the wave advanced directly across the Nemaha River and Salt Creek region. The thunderstorm activity was intensified by the development of a cyclonic circulation near the tip of the wave. At several recorder stations, these were the heaviest rains of the storm period and occurred between $5 \mathrm{p} . \mathrm{m}$. and midnight of the 8th. Dawson, for example, reported 2.23 inches in the 3 hours from 9 to 12 p.m. Of the 4.17 inches recorded at Pawnee City, 1.68 inches fell in a single hour, from 10 to $11 \mathrm{p} . \mathrm{m}$. Syracuse showed a total of 5.59 inches for the 5 -hour period from 5 to 10 p. m. More than 2 inches fell in the second hour of this period, and 1.85 inches fell in the last hour.

As the cold front crossed the southeastern corner of Nebraska, the rains at many stations continued beyond midnight into the early norning of the 9 th.

Storm of June 2, 1950, in the basins of Shell Creek and tributaries of Elkhorn River.

The precipitation that occurred over northeastern Nebraska in the early hours of June 2 was in the form of showers and thunder -torms, preceding and accompanying the passage of a cold front.

At 12:30 a. m., local time, June 1, a high-pressure area cen"ered over southwestern Missouri was bringing a deep layer of "varm moist air up from the Gulf of Mexico. Sever al thunder7torms occurring in the southern part of that State attested to the "nstability of the incoming air. The circulation over northeastern 'Tebraska, however, under the influence of a strong low in southsentral Canada, was from the west.

Twelve hours later, as the Missouri high-pressure area moved sastward, the circulation over Nebraska shifted so that it came irom the south, and dewpoints started to rise. Showers were berinning in eastern Kansas and western Missouri. At 6:30 p.m. on the 1st, a cold front began developing about 250 miles northwest of 'Torfolk (Madison County), Nebr. Clouds were building up to con$\rightarrow$ iderable heights in advance of the front. 
In another 6 hours the cold front had moved within 100 miles of Norfolk. Thunderstorms broke out in northeastern Nebraska where the flow of air was now from the southwest. Showers anc' thunderstorms were also reported behind the front. As the front continued its slow advance, thunderstorms persisted in the northeastern corner of Nebraska. By 6:30 a. m. June 2, the cold front was just past Norfolk, and the flow of air at the surface was from the northwest. During the 6-hour period from 12:30 a.m.to 6:30 a. m. 1.95 inches of rain fell at Norfolk. More than half of this amount fell between 3 and 4 a.m., e.s.t.

Meadow Grove and Madison, also in Madison County, received 1.47 inches and 3.29 inches of rainfall, respectively. In Platte County, Columbus received 1.14 inches, and Humphrey received 3. 95 inches. Albion in Boone County received 2.94 inches.

It should be noted that in a thunderstorm like this, only by chance would the heaviest rainfall occur at an official measuring station.

Showers and thunderstorms continued in some parts of northeastern Nebraska for another 3 hours. The winds to greater depths became northwesterly and all precipitation ended in northeastern Nebraska.

Storm of July 8-9, 1950, centered around York, Nebr.

One of the prime factors causing the heavy rains in eastern Nebraska was the flow of warm moist air in great depth from the Gulf of Mexico throughout the storm period and for a full day preceding it. Another important contributing factor was the cyclonic circulation that developed near the storm area.

During the entire storm period and the day before, a large high-pressure area dominated the eastern half of the United States. The air that flowed over eastern Nebraska had a long trajectory over the Gulf of Mexico from July 7 through the 9th. From early morning on the 8th to morning of the 9th, the flow of air tolevels as high as 10,000 feet was from a southerly or southwesterly direction, also bringing in moist air. A lee-of-the mountain trough, which had developed in eastern Montana early on the afternoon of the 6th, remained stationary until late on the 7 th, when it started moving eastward very slowly.

On the morning of July 8 the trough extended southward from the western border of North Dakota to eastern Colorado. Blocked by the high over the East, the trough continued to make exceedingly slow progress eastward for the next 24 hours. At 12:30 p.m., local 
time, a center of low pressure appeared to be forming in that part of the trough lying in western South Dakota. Scattered showers and thunderstorms had developed in eastern Nebraska by this time.

By 6 p. m. Grand Island, a bout 40 miles west of York, ha d received 2.91 inches of rain. The low that first appeared in western South Dakota had moved southward and now lay in western Nebraska. In the 2 hours from 7 to 9 p. m., 2. 60 inches of rain fell at York, 2. 30 inches of it having fallen in a single hour. In the next hour, from 9 to 10 p.m., an additional 4.99 inches was reported at York. These large amounts occurred as the trough neared York, having arrived just west of Grand Island at 8: 30 p. m. By this time the low, with its cyclonic circulation more pronounced, was in northwestern Kansas.

By midnight, York had accumulated a total of 11.59 inches of rainfall, Albion 4.21, Grand Island 4.65. Showers and thunderstorms continued through eastern Nebraska during the early morning hours of the 9 th. The circulation both at upper levels and the surface shifted on the 9 th, however, so that the air came from a more southeasterly direction with a trajectory over land, rather than from the Gulf, and was therefore drier.

By 6 a. $\mathrm{m}$. on the 9 th the heavy rains had stopped at most stations, although $t$ he trough continued its slow eastward movement. About noon the trough lay almost directly over York. During the next 12 hours the trough slowly retreated to its original position on the western border of Nebraska. Although drizzle was reported at a number of stations in eastern Nebraska during this time, the torrential rains were over.

\section{Storm of July 18,1950 , over the Loup River basin}

The rains that fell over the Loup River basin on July $18 \mathrm{re}$ sulted from instability in the warm air over that region. A cyclonic development to the southwest of the area was a contributing factor.

Early on the afternoon of the 17th a high that was centered of $f$ the Georgia coast dominated the southeastern United States as far west as Arkansas. A cold front extended from a low north of Lake Michigan southwestward through the northwest corner of Missouri, thence across central Kansas and the Texas Panhandle into a small low in southeastern New Mexico. Behind the front a high, which had moved down from Montana, was centered over western Nebraska. Winds over the Loup River basin were light and skies were clear. 
By midnight (local time) on the 17th, the low in New Mexico had deepened somewhat and the high had moved east of Nebraska. The nor thward flow of air on the east side of the low became stronger, with the result that the front began retreating as a warm front. Thunder storms and showers broke out in the Texas Panhandle, in $\mathrm{New}$ Mexico and Kansas; lightning was reported in Nebraska at Burwell. In the early morning hours of the 18th, thunderstorms arose in southern and central Nebraska as the warm front moved north ward into the southern part of the State. A $\mathrm{s}$ mall new low appeared in western Nebraska at this time. By 6:30 a. m. on the $18 \mathrm{th}$, rain was reported throughout the State north of the front that lay just south of the Loup River basin.

As the day progressed, the low in New Mexico extended itself northward until it finally merged with the low in western Nebraska. The increased cyclonic turning of the winds over the Loup River basin added to the instability of the air, and intensified the thunderstorm and shower activity. By noon Albion reported 2.14 inches of rain, and Spalding reported 2.13 inches.

Early in the afternoon the merged low started moving slowly eastward, and thunderstorms continued until the low arrived east of the Loup River basin about 9 p. $m$. By thistime Ericson. Greeley, and Loup City had rainfalls of more than 2 inches. Arcadia, Comstock, Genoa, Loup City, and Ordhad each received more than $1 \frac{1}{2}$ inches.

The high-pressure area again moved into central Nebraska from the northwest during the night, and by morning of the 19th winds had become light, and the skies cleared.

\section{MEASUREMENT OF FLOOD DISCHARGES}

Operations at gaging stations consist principally in deter mining a stage-discharge relation from which, under the conditions of a stable channel and a known stage, the discharge can be calculated. Many gaging stations in Nebraska are on streams with sandy beds, and because of almost continuous scour and fill the relation between stage and discharge is unstable. In order to determine the discharge for any known stage it therefore becomes necessary to make adjustments to the stage by an amount equal to the average scour or fill indicated by frequent current-meter discharge measurements.

Owing to widespread overflow or other adverse conditions related to the floods it was not possible to obtain current-meter measurements at all gaging stations or at all points at which measurement of peak discharge was desired. Determinations of maximum flow at $\mathrm{such}$ places have been based chiefly on 
computation of flow from slope-area observations, computation of flow through contracted openings, computation of flow over highway or railway embankments, and, at gaging stations, by extension of the rating curve. Examples of and general information about the use of these methods, with special references to the conditions experienced by the Geological Survey in applying them to various severe floods, a re given in Water -Supply Papers 773-E, 796-G, 798, 799, 800, 816, 843, and 888.

\section{STAGES AND DISCHARGES AT STREAM-GAGING STATIONS}

\section{Explanation of data}

The basic data systematically collected at $\mathrm{s} t \mathrm{r}$ e a $\mathrm{m}$-gaging stations consist of records of stage, measurements of discharge, and general information useful in determining the daily flow from the records of gage heights and discharge measurements. The records of stage are obtained either by periodic di $r$ e $t$ readings on a nonrecording gage or by a water-stage recorder that provides a continuous graph of stage. Measurements of discharge are generally made by a current meter. Occasionally, determination of extraordinary peak flows must be made by auxiliary methods referred to in the preceding section of this report.

The data tabulated on the following pages for each streamgaging station are as follows: a description of the station; a table showing the daily discharges throughout the 3-month period May to July 1950; and a table showing the stage and discharge at the indicated time during the periods of major floodflow, in sufficient detail to permit reliable definition of the flood hydrograph. The data are intended to include all essential information, although they are presented in concise form.

The description of the station gives information concerning the type, location, and datum of the gage, the area of the drainage basin, infor mation concerning the gage-height and discharge records, information on maxima, and pertinent miscellaneous remarks. A statement regarding the stage-discharge relation explains briefly the method used in defining the rating curve throughout the range of stage occurring in the floods of May-July 1950, as well as in the previous maximum flood of record, and gives information on conditions that affected the stage-discharge relation. The description also includes information about other auxiliary methods used in obtaining the discharge. The maximum stage and discharge at the gaging stationare given for the floods of May-July 1950 and for the indicated period of record prior thereto, and at some stations for floods antedating such period of record. 
The table of daily discharges presents the data generally for May, June, and July, which cover not only the period of the flood but also a time of sufficient length before and after the peak to show the relation of the flood discharges to the discharges of the preceding and following periods, thus giving a general perspective of the stream-flow conditions before, during, and after the flood rise. The table shows the monthly mean discharge for the 3 months, and the volume of runoff expressed in acre-feet.

The tables showing stage and discharge at indicated times are designed to present the rise and recession of the floods in detail. Not all stations were affected by each flood, and therefore the tables are not uniform in time.

Gage heights were obtained from records of continuous waterstage recorders, so far as such records were available. For stations at which the records were incomplete because of failure of the recording system, the method of computing the missing periods is explained in the section of the description concerning gage heights or discharge. For stations at which the gage-height record consisted of only one or more manual gage readings per day, the stages at indicated times generally represent such readings, unless otherwise explained.

Records are presented in downstream order. Records for gaging stations on the main streams are presented first, in downstream order, and then stations ontributaries in similar order beginning with the uppermost. Records of gaging stations published in this report relate to streams on which floods occurred and for those streams on the fringe of the flooded area. 


\section{Missouri River main stem}

Missouri River at Omaha, Nebr.

Location. --Lat $41^{\circ} 15^{\prime} 40^{\prime \prime}$, long. $95^{\circ} 55^{\prime} 15^{\prime \prime}$, in sec. 23, T. 15 N., R. 13 E., at Ak-Sar-Ben Bridge in Omaha. Datum of gage is $958.24 \mathrm{ft}$ above mean sea level, datum of 1929 .

Drainage area. $--322,800 \mathrm{sq} \mathrm{mi}$.

Gage-height record. --Water-stage recorder graph.

Discharge record. --Stage-discharge relation defined by current-meter measurements. Shifting-control method used.

Maxima. --May-July 1950: Discharge, 96, 900 cfs 3 a. $\mathrm{m}$. June 20 (gage height, $14.00 \mathrm{ft}$ ).

1928 to April 1950: Discharge, 200, 000 cfs Apr. 12, 1943; gage height, 22. 45 ft April $13,1943$.

Maximum stage known, $24.65 \mathrm{ft}$, present datum, Apr, 25, 1881 (ice jam).

Remarks. --Flow partly regulated by Fort Peck Reservoir.

Mean discharge, in cubic feet per second, 1950

\begin{tabular}{|c|c|c|c|c|c|c|c|c|c|c|c|}
\hline Day & May & June & July & Day & May & June & July & Day & May & June & July \\
\hline $\begin{array}{r}1 \\
2 \\
3 \\
4 \\
5 \\
6 \\
7 \\
8 \\
9 \\
10\end{array}$ & $\begin{array}{l}70,300 \\
63,200 \\
58,900 \\
55,100 \\
54,800 \\
54,400 \\
51,400 \\
51,400 \\
57,600 \\
58,500\end{array}$ & $\begin{array}{l}33,900 \\
39,400 \\
33,900 \\
32,500 \\
33,600 \\
33,600 \\
31,800 \\
30,700 \\
30,700 \\
31,800\end{array}$ & $\begin{array}{l}63,600 \\
59,800 \\
60,600 \\
68,500 \\
69,400 \\
59,300 \\
53,200 \\
50,600 \\
52,500 \\
47,600\end{array}$ & $\begin{array}{l}11 \\
12 \\
13 \\
14 \\
15 \\
16 \\
17 \\
18 \\
19 \\
20\end{array}$ & $\begin{array}{l}\mathbf{5 9}, \mathbf{8 0 0} \\
78,500 \\
79,500 \\
71,600 \\
\mathbf{6 7}, 600 \\
\mathbf{6 2}, 700 \\
\mathbf{5 6}, 800 \\
\mathbf{5 3}, 600 \\
\mathbf{5 2}, 900 \\
\mathbf{5 7}, 600\end{array}$ & $\begin{array}{l}34,300 \\
41,200 \\
45,800 \\
35,700 \\
39,800 \\
48,400 \\
43,900 \\
63,200 \\
91,200 \\
92,400\end{array}$ & $\begin{array}{l}49,100 \\
58,900 \\
59,300 \\
51,400 \\
53,600 \\
62,300 \\
54,000 \\
53,200 \\
55,100 \\
52,500\end{array}$ & $\begin{array}{l}21 \\
22 \\
23 \\
24 \\
25 \\
26 \\
27 \\
28 \\
29 \\
30 \\
31\end{array}$ & $\begin{array}{l}59,300 \\
50,600 \\
47,600 \\
45,000 \\
47,600 \\
41,600 \\
41,200 \\
38,300 \\
38,300 \\
38,300 \\
35,400\end{array}$ & $\begin{array}{c}68,000 \\
57,600 \\
52,900 \\
49,100 \\
45,400 \\
47,200 \\
61,900 \\
68,000 \\
71,600 \\
69,400 \\
-\end{array}$ & $\begin{array}{l}\mathbf{5 5}, \mathbf{9 0 0} \\
\mathbf{5 8}, 000 \\
\mathbf{5 3}, \mathbf{2 0 0} \\
\mathbf{5 0}, 600 \\
\mathbf{5 2}, 100 \\
49,100 \\
46,900 \\
44,600 \\
42,800 \\
41,200 \\
40,900\end{array}$ \\
\hline \multicolumn{9}{|c|}{ 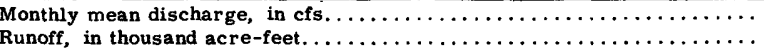 } & $\begin{array}{r}54,820 \\
3,371\end{array}$ & $\begin{array}{r}48,630 \\
2,894\end{array}$ & $\begin{array}{r}53,860 \\
3,312\end{array}$ \\
\hline
\end{tabular}

Missouri River at Nebraska City, Nebr.

Location. --Lat $40^{\circ} 40^{\prime} 35^{\prime \prime}$, long. $95^{\circ} 50^{\prime} 10^{\prime \prime}$, in SW $\frac{1}{4}$ sec. 10, T. 8 N., R. 14 E., at Waubonsie Highway bridge at Nebraska City. Datum of gage is $903.94 \mathrm{ft}$ above mean sea level, datum of 1929.

Drainage area. $--414,400 \mathrm{sq} \mathrm{mi}$.

Gage-height record.--Water-stage recorder graph.

Discharge record. --Stage-discharge relation defined by current-meter measurements. Shifting-control method used.

Maxima. --May-July 1950: Discharge, 147, 000 cfs 4:30 a. m. May 9 (gage height, $18.60 \mathrm{ft}$ ). 1929 to April 1950: Discharge, 214, 000 cfs June 14, 1944; gage height, 25. 8 ft Mar. 6, 1949 (ice jam).

Remarks. --Flow partly regulated by Fort Peck Reservoir.

Mean discharge, in cubic feet per second, 1950

\begin{tabular}{|c|c|c|c|c|c|c|c|c|c|c|c|}
\hline Day & May & June & July & Day & May & June & July & Day & May & June & July \\
\hline $\begin{array}{r}1 \\
2 \\
3 \\
4 \\
5 \\
6 \\
7 \\
8 \\
9 \\
10\end{array}$ & $\begin{array}{r}90,300 \\
77,200 \\
70,800 \\
65,100 \\
67,200 \\
62,500 \\
59,600 \\
58,600 \\
117,000 \\
88,700\end{array}$ & $\begin{array}{l}42,300 \\
42,800 \\
50,100 \\
53,600 \\
52,300 \\
50,100 \\
44,400 \\
39,800 \\
41,000 \\
38,100\end{array}$ & $\begin{array}{l}68,200 \\
62,000 \\
59,600 \\
63,500 \\
68,800 \\
62,500 \\
57,600 \\
54,400 \\
55,400 \\
57,200\end{array}$ & $\begin{array}{l}11 \\
12 \\
13 \\
14 \\
15 \\
16 \\
17 \\
18 \\
19 \\
20\end{array}$ & $\begin{array}{l}74,500 \\
88,700 \\
96,100 \\
88,700 \\
80,800 \\
74,500 \\
68,200 \\
63,500 \\
61,500 \\
64,000\end{array}$ & $\begin{array}{l}39,800 \\
40,600 \\
58,600 \\
43,600 \\
41,900 \\
47,000 \\
47,000 \\
51,400 \\
89,800 \\
98,800\end{array}$ & $\begin{array}{l}70,300 \\
82,900 \\
84,000 \\
65,100 \\
61,000 \\
77,200 \\
70,800 \\
62,500 \\
71,900 \\
98,300\end{array}$ & $\begin{array}{l}21 \\
22 \\
23 \\
24 \\
25 \\
26 \\
27 \\
28 \\
29 \\
30 \\
31\end{array}$ & $\begin{array}{l}75,000 \\
70,300 \\
57,200 \\
54,000 \\
58,100 \\
52,700 \\
49,200 \\
44,400 \\
44,400 \\
47,900 \\
45,300\end{array}$ & $\begin{array}{c}84,000 \\
66,100 \\
63,000 \\
57,200 \\
49,600 \\
47,000 \\
56,200 \\
66,600 \\
73,000 \\
74,000 \\
-\end{array}$ & $\begin{array}{l}81,900 \\
78,700 \\
75,000 \\
65,600 \\
71,400 \\
64,000 \\
57,200 \\
53,100 \\
51,000 \\
48,300 \\
51,000\end{array}$ \\
\hline \multicolumn{9}{|c|}{ 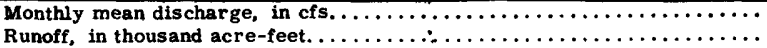 } & $\begin{array}{r}68,260 \\
4,197\end{array}$ & $\begin{array}{r}54,990 \\
3,272\end{array}$ & $\begin{array}{r}66,140 \\
4,067\end{array}$ \\
\hline
\end{tabular}


Missouri River at Rulo, Nebr.

Location. --Lat $40^{\circ} 03^{\prime} 15^{\prime \prime}$, long. $95^{\circ} 25^{\prime} 15^{\prime \prime}$, in NW $\frac{1}{4} \mathrm{NW} \frac{1}{4}$ sec. $17, T$. 1 N., R. $18 \mathrm{E}$., at C., B. \& $Q$. R. R. bridge at Rulo, $80 \mathrm{ft}$ upstream from U. S. Highway 159 bridge, 3.2 miles upstream from Nemaha River, and 514.4 miles above mouth (1932 mileage). Datum of gage is $837.23 \mathrm{ft}$ above mean sea level, datum of 1929 .

Drainage area. $--418,905 \mathrm{sq} \mathrm{mi}$.

Gage-height record.--Graph drawn on basis of once-daily wire-weight gage readings and hourly gage readings during flood period of May 9 and 10. Crest gage height was observed.

Discharge record. --Stage-discharge relation defined by current-meter measurements made at approximately 5-day intervals. Shifting-control method used throughout. Gage heights used to half tenths.

Maxima. --May-July 1950: Discharge, 172,000 cfs 6 a. m. May 10 (gage height, $21.55 \mathrm{ft}$ ).

1949 to July 1950: Discharge, 185,000 cfs from 8 a.m. to 2 p.m. Apr. 29, 1950; gage height, $21.6 \mathrm{ft}$ from 8 a.m. to 10 a.m. Apr. 30, 1950 .

Remarks. --Flow partly regulated by many reservoirs in the basin.

Mean discharge, in cubic feet per second, 1950

\begin{tabular}{|c|c|c|c|c|c|c|c|c|c|c|c|}
\hline Day & May & June & July & Day & I May & June & July & Day & May & June & July \\
\hline $\begin{array}{r}1 \\
2 \\
3 \\
4 \\
5 \\
6 \\
7 \\
8 \\
9 \\
10\end{array}$ & $\begin{array}{r}118,000 \\
93,400 \\
76,300 \\
72,000 \\
70,600 \\
71,000 \\
67,400 \\
67,800 \\
137,000 \\
162,000\end{array}$ & $\begin{array}{l}47,600 \\
43,800 \\
50,500 \\
47,200 \\
55,400 \\
52,400 \\
49,400 \\
43,400 \\
56,100 \\
41,600\end{array}$ & $\begin{array}{l}78, \\
70, \\
64, \\
67, \\
74, \\
73, \\
64, \\
58, \\
56, \\
59,\end{array}$ & $\begin{array}{l}11 \\
12 \\
13 \\
14 \\
15 \\
16 \\
17 \\
18 \\
19 \\
20\end{array}$ & $\begin{array}{r}116,000 \\
89,700 \\
106,000 \\
100,000 \\
88,700 \\
82,200 \\
74,800 \\
69,200 \\
65,600 \\
66,000\end{array}$ & $\begin{array}{r}39,000 \\
38,000 \\
39,300 \\
56,100 \\
44,400 \\
46,600 \\
51,600 \\
49,400 \\
85,200 \\
101,000\end{array}$ & $\begin{array}{r}65,600 \\
69,200 \\
101,000 \\
82,700 \\
66,000 \\
85,200 \\
87,700 \\
74,400 \\
64,300 \\
80,700\end{array}$ & $\begin{array}{l}21 \\
22 \\
23 \\
24 \\
25 \\
26 \\
27 \\
28 \\
29 \\
30 \\
31\end{array}$ & $\begin{array}{l}78, \\
78, \\
69, \\
63, \\
67, \\
68, \\
58, \\
50, \\
52, \\
58,\end{array}$ & $\begin{array}{c}92,900 \\
75,800 \\
64,700 \\
63,800 \\
59,700 \\
51,600 \\
53,500 \\
70,100 \\
79,700 \\
83,700 \\
-\end{array}$ & $\begin{array}{l}89,700 \\
77,200 \\
74,400 \\
68,800 \\
66,500 \\
73,900 \\
63,400 \\
57,300 \\
52,800 \\
49,800 \\
50,500\end{array}$ \\
\hline \multicolumn{9}{|c|}{$\begin{array}{l}\text { Monthly mean discharge, in cfs. } \ldots \ldots \ldots \ldots \ldots \ldots \ldots \ldots \ldots \ldots \ldots \ldots \ldots \ldots \ldots \ldots \\
\text { Runoff, in thousand acre-feet. } \ldots \ldots \ldots \ldots \ldots \ldots \ldots \ldots \ldots \ldots\end{array}$} & $\begin{array}{r}80,450 \\
4,947\end{array}$ & $\begin{array}{r}57,780 \\
3,438\end{array}$ & $\begin{array}{r}69,990 \\
4,303\end{array}$ \\
\hline
\end{tabular}

\section{Platte River basin}

Platte River near Grand Island, Nebr.

Location. --Lat $40^{\circ} 53^{\prime}$, long. $98^{\circ} 17$ ', in sec. 36, T. 11 N., R. 9 W., at bridge on U. S. Highway 34, 2 miles upstream from Chicago, Burlington \& Quincy Railroad bridge and 5 miles southeast of Grand Island.

Drainage area. $--59,500 \mathrm{sq} \mathrm{mi}$.

Gage-height record. --Water-stage recorder graph. $\frac{\text { Discharge record. --Stage-discharge relation defined by current-meter measurements. Shifting-control }}{\text { method used. }}$

Maxima. -May-July 1950: Discharge, 4, $310 \mathrm{cfs} 3: 30$ a. m. May 9 (gage height, 3.60 ft).

1933 to April 1950: Discharge, 30,000 cfs June 6, 1935, from rating curve extended above $14,000 \mathrm{cfs}$; gage height, 6.03 ft Mar. 4, 1949 (backwater from ice).

Remarks. --Natural flow of stream affected by transmountain diversions, storage reservoirs, power developments, ground-water withdrawals and diversions for irrigation, and return flow from irrigated areas.

Mean discharge, in cubic feet per second, 1950

\begin{tabular}{|c|c|c|c|c|c|c|c|c|c|c|c|}
\hline Day & May & June & July & Day & May & June & July & Day & May & June & July \\
\hline $\begin{array}{r}1 \\
2 \\
3 \\
4 \\
5 \\
6 \\
7 \\
8 \\
9 \\
10\end{array}$ & $\begin{array}{r}1,290 \\
1,100 \\
859 \\
822 \\
1,050 \\
1,220 \\
1,200 \\
2,600 \\
3,670 \\
2,710\end{array}$ & $\begin{array}{r}2,490 \\
1,960 \\
1,550 \\
1,480 \\
1,310 \\
1,200 \\
1,140 \\
896 \\
616 \\
525\end{array}$ & $\begin{array}{r}199 \\
183 \\
302 \\
234 \\
335 \\
175 \\
136 \\
147 \\
1,500 \\
1,670\end{array}$ & $\begin{array}{l}11 \\
12 \\
13 \\
14 \\
15 \\
16 \\
17 \\
18 \\
19 \\
20\end{array}$ & $\begin{array}{l}2,280 \\
2,340 \\
2,280 \\
1,720 \\
1,570 \\
1,250 \\
1,100 \\
1,200 \\
1,520 \\
1,400\end{array}$ & $\begin{array}{l}466 \\
386 \\
273 \\
412 \\
412 \\
495 \\
649 \\
766 \\
525 \\
466\end{array}$ & \begin{tabular}{l||}
896 \\
569 \\
438 \\
386 \\
293 \\
253 \\
412 \\
348 \\
302 \\
244
\end{tabular} & $\begin{array}{l}21 \\
22 \\
23 \\
24 \\
25 \\
26 \\
27 \\
28 \\
29 \\
30 \\
31 \\
\end{array}$ & $\begin{array}{l}1,480 \\
1,520 \\
1,380 \\
1,100 \\
1,080 \\
1,100 \\
1,220 \\
1,570 \\
2,740 \\
2,580 \\
3,000 \\
\end{array}$ & $\begin{array}{c}466 \\
569 \\
510 \\
425 \\
466 \\
466 \\
466 \\
412 \\
335 \\
263 \\
- \\
\end{array}$ & $\begin{array}{r}273 \\
244 \\
904 \\
1,220 \\
915 \\
749 \\
733 \\
822 \\
803 \\
699 \\
840 \\
\end{array}$ \\
\hline \multicolumn{9}{|c|}{$\begin{array}{l}\text { Monthly mean discharge, in cfa } \ldots \ldots \ldots \ldots \ldots \ldots \ldots \ldots \ldots \ldots \ldots \ldots \ldots \ldots \ldots \ldots \ldots \\
\text { Runoff, in acre-feet. } \ldots \ldots \ldots \ldots \ldots \ldots \ldots \ldots \ldots \ldots \ldots \ldots \ldots \ldots \ldots \ldots \ldots \ldots \ldots \\
\end{array}$} & $\begin{array}{r}1,676 \\
103,000\end{array}$ & $\begin{array}{r}746 \\
44,420\end{array}$ & $\begin{array}{r}556 \\
34,160 \\
\end{array}$ \\
\hline
\end{tabular}


PLATTE RIVER BASIN

Platte River near Grand Island, Nebr. -Continued

Gage height, in feet, and discharge, in cubic feet per second, at indicated time, 1950

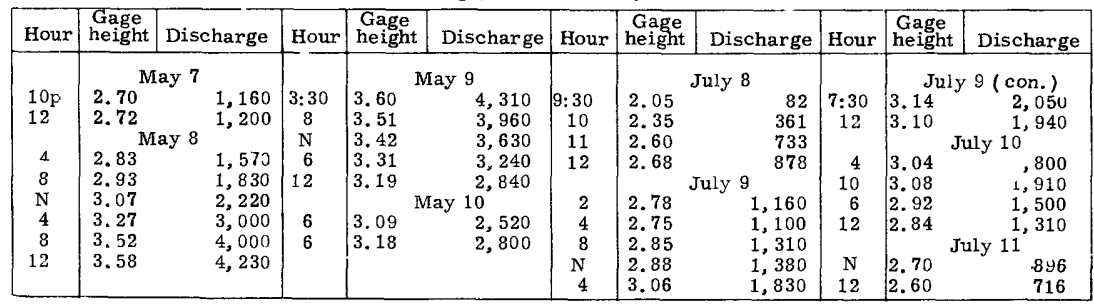

Platte River near Duncan, Nebr.

Location. --Lat $41^{\circ} 22^{\prime}$, long. $97^{\circ} 29^{\prime}$, in sec. 12, T. 16 N., R. 2 W., at highway bridge, $1 \frac{1}{2}$ miles south of Duncan and 12 miles upstream from Loup River. Datum of gage is 1,478.55 $\mathrm{ft}$ above mean sea level, datum of 1929 .

Drainage area. $--61,600 \mathrm{sq} \mathrm{mi}$.

Gage-height record. - Water-stage recorder graph except 5 a. m. May 6 to 6 p. m. May 7 when there was no gage-height record, and July 10, 11 when graph was partly estimated.

Discharge record. --Stage-discharge relation defined by current-meter measurements. Shifting-control method used.

Maxima. --May-July 1950: Discharge, 4, $150 \mathrm{cfs} 1: 15$ p. m. May 10 (gage height, $3.10 \mathrm{ft}$ ). 1895-1915, 1928 to April 1950: Discharge observed, 51, 100 cfs June 23, 1905.

Remarks. --Natural flow of stream affected by transmountain diversions, storage reservoirs, power developments, ground-water withdrawals and diversions for irrigation, and return flow from irrigated areas.

Mean discharge, in cubic feet per second, 1950

\begin{tabular}{|c|c|c|c|c|c|c|c|c|c|c|c|}
\hline Day & May & June & July & Day & May & June & July & Day & May & June & July \\
\hline 1 & 1,290 & 3,000 & 261 & 11 & 3,170 & 621 & 2,200 & 21 & 1,850 & 550 & 643 \\
\hline 2 & 1,430 & 3,020 & 214 & 12 & 2,740 & 502 & 2,240 & 22 & 1,910 & 442 & 621 \\
\hline 3 & 1,340 & 2,350 & 273 & 13 & 2,520 & 350 & 1,910 & 23 & 1,750 & 402 & 590 \\
\hline 4 & 1,020 & 1,910 & 298 & 14 & 2,390 & 330 & 1980 & 24 & 1,810 & 493 & 676 \\
\hline 5 & 1,070 & 1,640 & 285 & 15 & 2,100 & 402 & 1,680 & 25 & 1,550 & 493 & 1,500 \\
\hline 6 & 1,200 & 1,380 & 198 & 16 & 1,870 & 395 & 1,270 & 26 & 1,530 & 324 & 1,530 \\
\hline 7 & 1,400 & 1,240 & 208 & 17 & 1,430 & 468 & 1,020 & 27 & 1,510 & 350 & 1,410 \\
\hline 8 & 1,980 & 832 & 160 & 18 & 1,320 & 698 & 953 & 28 & 1,550 & 372 & 1,360 \\
\hline 9 & 2,410 & 808 & 600 & 19 & 1,500 & 676 & 1,020 & 29 & 1,830 & 395 & 1,130 \\
\hline 10 & 3 , & 720 & 1,680 & 20 & 1,700 & 709 & 858 & 30 & 2,350 & 318 & 1,150 \\
\hline \multicolumn{9}{|c|}{ 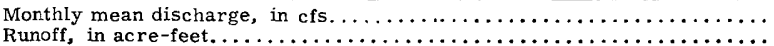 } & $\begin{array}{r}1,885 \\
115,900\end{array}$ & $\begin{array}{r}873 \\
51,950\end{array}$ & $\begin{array}{r}1,000 \\
61,460\end{array}$ \\
\hline
\end{tabular}

Gage height, in feet, and discharge, in cubic feet per second, at indicated time, 1950

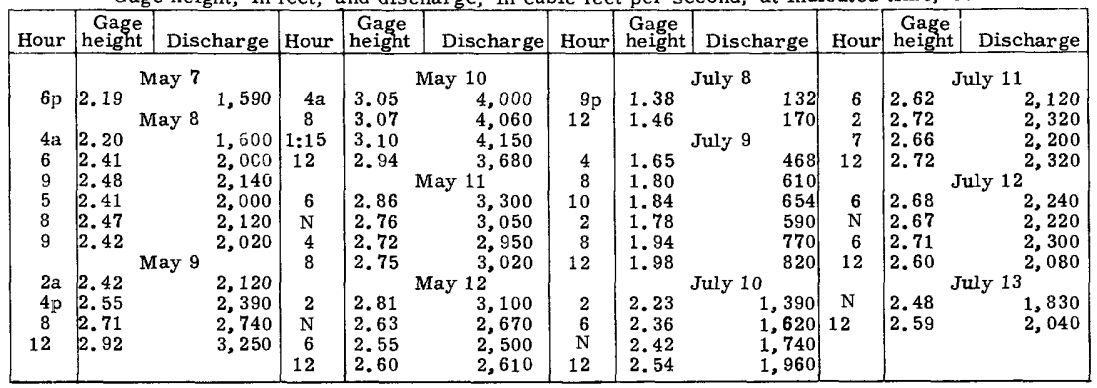

238744 O $-53-5$ 
Platte River at North Bend, Nebr.

Location. --Lat $41^{\circ} 27^{\prime} 10^{\prime \prime}$, long. $96^{\circ} 45^{\prime} 50^{\prime \prime}$, in $\mathrm{SE} \frac{1}{4} \mathrm{sec}, 7, \mathrm{~T}, 17 \mathrm{~N} .$, R. 6 E., at bridge on State Highway 79, 1 mile south of North Bend and 5 miles downstream from Shell Creek.

Gage-height record. --Graph drawn on basis of twice-daily wire-weight gage readings and occasional readings by engineers.

Discharge records. --Stage-discharge relation defined by current-meter measurements below 19,000 cfs and extended to peak stage. Shifting-control method used.

Maxima. --May-July 1950: Discharge, 25, 000 cfs 5 p. m. July 12 (gage height, $5.3 \mathrm{ft}$, from graph based on gage readings).

April 1949 to April 1950: Discharge, 21, 000 cfs June 2, 1949; gage height, 6. 0 ft March 8, 1950, from graph based on gage readings (backwater from ice).

On June 24, 1947 peak discharge was about $90,000 \mathrm{cfs}$ (estimated on basis of peak discharge near Ashland and Loup River at Columbus).

Remarks. - Natural flow of stream affected by transmountain diversions, storage reservoirs, power developments, ground water withdrawals and diversions for irrigation, and return flow from irrigated areas. Diurnal fluctuations from power plant on Loup River.

Mean discharge, in cubic feet per second, 1950

\begin{tabular}{|c|c|c|c|c|c|c|c|c|c|c|c|}
\hline Day & May & June & July & Day & May & June & July & Day & May & June & July \\
\hline $\begin{array}{r}1 \\
2 \\
3 \\
4 \\
5 \\
6 \\
7 \\
8 \\
9 \\
9 \\
10\end{array}$ & $\begin{array}{l}4,070 \\
4,160 \\
3,700 \\
3,700 \\
4,070 \\
3,730 \\
4,340 \\
5,640 \\
6,920 \\
6,960\end{array}$ & $\begin{array}{r}5,290 \\
5,220 \\
10,700 \\
8,790 \\
7,240 \\
4,550 \\
3,540 \\
3,400 \\
3,090 \\
2,720\end{array}$ & \begin{tabular}{r|}
2,790 \\
2,670 \\
1,830 \\
2,040 \\
2,420 \\
2,580 \\
2,420 \\
2,420 \\
3,040 \\
16,900
\end{tabular} & $\begin{array}{l}11 \\
12 \\
13 \\
14 \\
15 \\
16 \\
17 \\
18 \\
19 \\
20\end{array}$ & $\begin{array}{l}7,000 \\
6,020 \\
5,540 \\
5,460 \\
5,540 \\
4,940 \\
5,000 \\
4,970 \\
4,610 \\
7,360\end{array}$ & $\begin{array}{l}2,990 \\
2,540 \\
2,720 \\
2,200 \\
2,420 \\
2,870 \\
2,990 \\
3,170 \\
3,450 \\
4,520\end{array}$ & $\begin{array}{r}12,500 \\
15,000 \\
12,700 \\
9,250 . \\
7,320 \\
6,280 \\
6,320 \\
9,750 \\
19,600 \\
13,900\end{array}$ & $\begin{array}{l}21 \\
22 \\
23 \\
24 \\
25 \\
26 \\
27 \\
28 \\
29 \\
30 \\
31\end{array}$ & $\begin{array}{l}7,080 \\
5,120 \\
4,850 \\
4,610 \\
4,430 \\
4,370 \\
4,250 \\
4,760 \\
5,180 \\
5,180 \\
5,500\end{array}$ & $\begin{array}{l}3,670 \\
2,870 \\
2,630 \\
2,540 \\
2,490 \\
2,220 \\
2,310 \\
2,100 \\
2,140 \\
2,580 \\
-\end{array}$ & $\begin{array}{r}9,800 \\
8,480 \\
7,280 \\
10,700 \\
9,850 \\
8,120 \\
5,710 \\
5,120 \\
4,280 \\
4,370 \\
3,560\end{array}$ \\
\hline \multicolumn{9}{|c|}{$\begin{array}{l}\text { Monthly mean discharge, in cfs } \ldots \ldots \ldots \ldots \ldots \ldots \ldots \ldots \ldots \ldots \ldots \ldots \ldots \ldots \ldots \ldots \ldots \ldots \\
\text { Runoff, in acre-feet. } \ldots \ldots \ldots \ldots \ldots \ldots \ldots \ldots \ldots \ldots \ldots \ldots \ldots \ldots \ldots \ldots \\
\end{array}$} & $\begin{array}{r}5,131 \\
315,500\end{array}$ & $\begin{array}{r}3,665 \\
218,100\end{array}$ & $\begin{array}{r}7,387 \\
454,200 \\
\end{array}$ \\
\hline
\end{tabular}

Gage height, in feet, and discharge, in cubic feet per second, at indicated time, 1950

\begin{tabular}{|c|c|c|c|c|c|c|c|c|c|c|c|}
\hline Hour & $\begin{array}{l}\text { Gage } \\
\text { height }\end{array}$ & Discharge & Hour & $\begin{array}{c}\text { Gage } \\
\text { height }\end{array}$ & Discharge & Hour & $\begin{array}{c}\text { Gage } \\
\text { height }\end{array}$ & Discharge & Hour & $\begin{array}{c}\text { Gage } \\
\text { height }\end{array}$ & Discharge \\
\hline & \multicolumn{2}{|c|}{ June 2} & & \multicolumn{2}{|r|}{ June 5} & & \multicolumn{2}{|c|}{ July 11} & & \multicolumn{2}{|c|}{ July 13 (Con.) } \\
\hline 12 & 2.13 & 4,340 & 2 & 2. 97 & 7,440 & 4 & 3.85 & 14,800 & 4 & 3.50 & 12,800 \\
\hline & & June 3 & 6 & 3.16 & 8,250 & 8 & 3.40 & 12,200 & 8 & 3.40 & 12,200 \\
\hline 4 & 3.18 & & 10 & 3.23 & 8,560 & $\mathrm{~N}$ & 3.29 & 11,600 & \multirow{3}{*}{12} & \multicolumn{2}{|c|}{ July 18} \\
\hline 8 & 3.94 & 12,100 & 2 & 2.89 & 7,120 & 4 & 3.23 & 11,300 & & \multirow{2}{*}{\multicolumn{2}{|c|}{.60}} \\
\hline 10 & 4.05 & 12,700 & $\overline{6}$ & 2.60 & 5,9 & 8 & 3.20 & 11,200 & & & \\
\hline 2 & 3.97 & 12,300 & 10 & 2.52 & 5,710 & 12 & 3.17 & 11,000 & 4 & 55 & 13,100 \\
\hline 6 & 4.00 & 12,500 & & & July 9 & & \multicolumn{2}{|c|}{ July 12} & 8 & 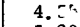 & 19,400 \\
\hline 10 & 3.83 & 11,500 & 12 & 1.40 & 2,380 & 4 & 3.17 & 11,000 & $\mathbf{N}$ & 5.20 & 24,200 \\
\hline & \multicolumn{2}{|c|}{ June 4} & & & July 10 & 8 & 3.17 & 11,000 & 4 & 5,22 & 24,400 \\
\hline 2 & 3.47 & 9,700 & 4 & 3.50 & 11,200 & $\mathbf{N}$ & 3.40 & 12,200 & 8 & 4.95 & 22,200 \\
\hline 6 & 3.42 & 9,450 & 8 & 5.10 & 23,400 & 5 & 5.30 & 25,000 & 12 & 4.55 & 19,400 \\
\hline 10 & 3.40 & 9,350 & $\mathbf{N}$ & 4.86 & 21,500 & 8 & 4.45 & 18,700 & & \multicolumn{2}{|c|}{ July 20} \\
\hline 2 & 3.27 & 8,740 & 4 & 4.52 & 19,100 & 12 & 3.35 & 12,000 & 4 & 4.22 & 17,200 \\
\hline 6 & 3.08 & 7,890 & 8 & 4.32 & & & \multicolumn{2}{|c|}{ July 13} & 8 & 3.90 & 15,200 \\
\hline 10 & 2.91 & 7,200 & 12 & 4.31 & 17,800 & 4 & 3.44 & 12,500 & $\mathrm{~N}$ & 3.65 & 13,600 \\
\hline & & & & & & 8 & 3.60 & 400 & 4 & 3.38 & $12,10 \theta$ \\
\hline & & & & & & $\mathrm{N}$ & 3.60 & 13,400 & 8 & 3.15 & 10,900 \\
\hline & & & & & & & & & 12 & 3.10 & 10,600 \\
\hline
\end{tabular}


Platte River near Ashland, Nebr.

Location. - Lat $41^{\circ} 03^{\prime} 30^{\prime \prime}$, long. $96^{\circ} 19^{\prime} 30^{\prime \prime}$, on line between secs. 29 and 32, T. 13 N., R. 10 E., at bridge on U. S. Highway 6,100 ft downstream from Chicago, Burlington \& Quincy Railroad bridge, 2 miles upstream from Salt Creek, and 3 miles northeast of Ashland. Datum of gage is $1,052.51 \mathrm{ft}$ above mean sea level, datum of 1929 .

Drainage area, $--83,800 \mathrm{sq} \mathrm{mi}$.

Gage-height record. --Water-stage recorder graph.

Discharge record. --Stage-discharge relation defined by current-meter measurements. Shifting-control method used.

Maxima. --May-July 1950: Discharge, 47, 900 cfs 2:30 a. m. July 20 (gage height, $6.30 \mathrm{ft}$ ). 1928 to April 1950: Discharge, $107,000 \mathrm{cfs} J u n e 12,1944$, includes overbank flow caused by breaking of dikes; gage height, $8.33 \mathrm{ft} \mathrm{Mar.} \mathrm{5,} 1936$ (site then in use), ice jam.

Flood of June 1944 is greatest since 1883 and possibly largest known.

Remarks. - Natural flow of stream affected by transmountain diversions, storage reservoirs, power developments, ground-water vithdrawals and diversion for irrigation, and return flow from irrigated areas.

Mean discharge, in cubic feet per second, 1950

\begin{tabular}{|c|c|c|c|c|c|c|c|c|c|c|c|}
\hline Day & May & June & July & Day & May & June & July & Day & May & June & July \\
\hline $\begin{array}{r}1 \\
2 \\
3 \\
4 \\
5 \\
6 \\
7 \\
8 \\
9 \\
10\end{array}$ & $\begin{array}{r}6,050 \\
6,450 \\
6,050 \\
5,590 \\
5,740 \\
5,440 \\
4,970 \\
5,550 \\
9,260 \\
13,300\end{array}$ & $\begin{array}{r}7,320 \\
7,190 \\
13,800 \\
17,900 \\
16,000 \\
12,500 \\
8,160 \\
6,940 \\
6,450 \\
5,590\end{array}$ & \begin{tabular}{r|}
3,000 \\
2,950 \\
2,870 \\
2,430 \\
2,480 \\
3,320 \\
2,820 \\
2,820 \\
3,320 \\
16,400
\end{tabular} & $\begin{array}{l}11 \\
12 \\
13 \\
14 \\
15 \\
16 \\
17 \\
18 \\
19 \\
20\end{array}$ & $\begin{array}{r}13,0 \\
12,3 \\
10,4 \\
8,8 \\
8,7 \\
8,1 \\
6,7 \\
6,5 \\
6,1 \\
6,2\end{array}$ & $\begin{array}{l}4, \\
4, \\
3, \\
4, \\
3, \\
3, \\
3, \\
4, \\
6, \\
7,\end{array}$ & $\begin{array}{r}14,400 \\
13,600 \\
18,800 \\
13,400 \\
10,500 \\
13,600 \\
9,890 \\
12,200 \\
22,800 \\
33,600\end{array}$ & $\begin{array}{l}23 \\
23 \\
24 \\
25 \\
26 \\
27 \\
28 \\
29 \\
30 \\
31 \\
\end{array}$ & $\begin{array}{r}10,900 \\
7,980 \\
7,720 \\
7,190 \\
6,940 \\
6,210 \\
5,900 \\
5,860 \\
6,130 \\
6,330 \\
6,980 \\
\end{array}$ & $\begin{array}{c}7,670 \\
6,820 \\
5,900 \\
4,830 \\
4,310 \\
4,150 \\
3,600 \\
3,320 \\
3,130 \\
3,160 \\
- \\
\end{array}$ & $\begin{array}{r}21,800 \\
20,800 \\
14,700 \\
17,100 \\
19,900 \\
14,500 \\
10,500 \\
9,370 \\
7,410 \\
6,210 \\
6,370 \\
\end{array}$ \\
\hline \multicolumn{9}{|c|}{ 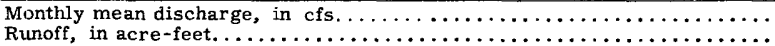 } & $\begin{array}{r}7,537 \\
463,500\end{array}$ & $\begin{array}{r}6,521 \\
388,000\end{array}$ & $\begin{array}{r}11,410 \\
701,900\end{array}$ \\
\hline
\end{tabular}

Gage height, in feet, and discharge, in cubic feet per second, at indicated time, 1950

\begin{tabular}{|c|c|c|c|c|c|c|c|c|c|c|c|}
\hline Hour & $\begin{array}{c}\begin{array}{c}\text { Gage } \\
\text { height }\end{array} \\
\end{array}$ & Discharge & Hour & \begin{tabular}{|c|} 
Gage \\
height
\end{tabular} & Discharge & Hour & $\begin{array}{c}\text { Gage } \\
\text { height }\end{array}$ & Discharge & Hour & \begin{tabular}{|c|} 
Gage \\
height
\end{tabular} & Discharge \\
\hline $\begin{array}{c}4 \mathbf{a} \\
5 \\
6 \\
7 \\
8 \\
9 \\
10 \\
11 \\
\mathrm{~N} \\
1 \\
2 \\
3 \\
4 \\
5 \\
6 \\
7 \\
8 \\
9 \\
10 \\
11 \\
12\end{array}$ & $\begin{array}{l}\text { J } \\
1.41 \\
1.42 \\
1.46 \\
2.18 \\
4.35 \\
4.74 \\
4.85 \\
4.91 \\
4.97 \\
5.00 \\
5.00 \\
5.00 \\
4.95 \\
4.90 \\
4.84 \\
4.75 \\
4.66 \\
4.59 \\
4.51 \\
4.44 \\
4.36\end{array}$ & $\begin{array}{r}10 \\
2,870 \\
2,900 \\
3,000 \\
5,480 \\
18,500 \\
21,900 \\
22,900 \\
23,400 \\
24,000 \\
24,300 \\
24,300 \\
24,300 \\
23,900 \\
23,400 \\
22,900 \\
22,200 \\
21,400 \\
20,800 \\
20,200 \\
19,600 \\
19,000\end{array}$ & $\begin{array}{r}2 \mathrm{a} \\
4 \\
6 \\
8 \\
10 \\
\mathrm{~N} \\
2 \\
4 \\
6 \\
8 \\
10 \\
12 \\
2 \\
2 \\
4 \\
6 \\
8 \\
10 \\
\mathrm{~N} \\
2 \\
4 \\
6 \\
8 \\
10 \\
12 \\
\end{array}$ & $\begin{array}{l}4.19 \\
3.99 \\
3.83 \\
3.74 \\
3.70 \\
3.67 \\
3.65 \\
3.61 \\
3.52 \\
3.42 \\
3.34 \\
3.25 \\
3.20 \\
3.24 \\
3.59 \\
3.81 \\
3.80 \\
3.78 \\
3.73 \\
3.68 \\
3.58 \\
3.43 \\
3.33 \\
4.36\end{array}$ & $\begin{array}{r}\text { July } 11 \\
17,700 \\
16,300 \\
15,200 \\
14,600 \\
14,400 \\
14,200 \\
14,000 \\
13,800 \\
13,200 \\
12,600 \\
12,100 \\
11,600 \\
\text { July } 12 \\
11,300 \\
11,500 \\
13,600 \\
15,100 \\
15,000 \\
14,900 \\
14,500 \\
14,200 \\
13,600 \\
12,700 \\
12,100 \\
19,000\end{array}$ & $\begin{array}{r}2 \\
4 \\
6 \\
8 \\
8 \\
10 \\
\mathrm{~N} \\
2 \\
4 \\
6 \\
8 \\
10 \\
12 \\
12 \\
\\
\mathrm{~N} \\
1 \\
2 \\
3 \\
4 \\
5 \\
6 \\
7 \\
8 \\
9\end{array}$ & $\begin{array}{l}5.14 \\
5.27 \\
5.01 \\
4.69 \\
4.44 \\
4.26 \\
4.02 \\
3.82 \\
3.68 \\
3.61 \\
3.56 \\
3.53 \\
3.56 \\
3.57 \\
3.57 \\
3.58 \\
3.80 \\
4.44 \\
4.82 \\
5.13 \\
5.33 \\
5.48\end{array}$ & $\begin{array}{r}\text { July } 13 \\
25,600 \\
26,800 \\
24,400 \\
21,600 \\
19,600 \\
18,300 \\
16,500 \\
15,100 \\
14,200 \\
13,800 \\
13,500 \\
13,300 \\
\text { July } 19 \\
15,800 \\
15,400 \\
15,900 \\
16,000 \\
17,500 \\
24,100 \\
29,000 \\
33,500 \\
36,100 \\
37,800\end{array}$ & \begin{tabular}{|c}
10 \\
11 \\
12 \\
\\
1 \\
2 \\
$2: 30$ \\
3 \\
4 \\
5 \\
6 \\
7 \\
8 \\
9 \\
10 \\
11 \\
$\mathrm{~N}$ \\
2 \\
4 \\
6 \\
8 \\
10 \\
12
\end{tabular} & $\begin{array}{l}\text { July } \\
5.70 \\
6.00 \\
6.11 \\
6.22 \\
6.29 \\
6.30 \\
6.29 \\
6.25 \\
6.17 \\
6.07 \\
5.93 \\
5.76 \\
5.60 \\
5.40 \\
5.18 \\
5.00 \\
4.68 \\
4.45 \\
4.32 \\
4.21 \\
4.15 \\
4.12\end{array}$ & $\begin{array}{r}9 \text { (con.) } \\
40,400 \\
44,100 \\
45,500 \\
\mathrm{y} 20 \\
46,900 \\
47,800 \\
47,900 \\
47,800 \\
47,300 \\
46,200 \\
45,000 \\
43,200 \\
41,100 \\
39,200 \\
36,900 \\
34,400 \\
31,900 \\
27,600 \\
24,800 \\
23,300 \\
22,200 \\
21,600 \\
21,400\end{array}$ \\
\hline
\end{tabular}


Wood River near Gibbon, Nebr.

Location. --Lat $40^{\circ} 46^{\prime} 10^{\prime \prime}$, long. $98^{\circ} 48^{\prime} 00^{\prime \prime}$, in NW $\frac{1}{4}$ sec. 9, T, 9 N., R. 13 W., at county bridge $2 \frac{1}{4}$ miles northeast of Gibbon.

Drainage area., $-\mathbf{- 5 7 2}$ sq $\mathrm{mi}$.

Gage-height record. --Water-stage recorder graph except for period 9 a. m. June 6 to 9 a. $m$. June 13 when no record was obtained.

Discharge record. --Stage-discharge relation defined by current-meter measurements. Shifting-control method used for low and medium stages May 1 to June 6, June 20 to July 4, July 11-31; rating curve used direct at high stages.

Maxima. --May-July 1950: Discharge, $1,680 \mathrm{cfs} 3$ to $5 \mathrm{p} . \mathrm{m}$. July 10 (gage height, 15. $74 \mathrm{ft}$ ). April 1949 to April 1950: Discharge, 1,600 cís June 9, 1949 (gage height, $15.63 \mathrm{ft}$ ).

Remarks. --Flow during flood period not affected by irrigation diversions.

Niean discharge, in cubic feet per second, 1950

\begin{tabular}{|c|c|c|c|c|c|c|c|c|c|c|c|}
\hline Day & May & June & July & Day & May & June & July & Day & May & June & July \\
\hline $\begin{array}{r}1 \\
2 \\
3 \\
4 \\
5 \\
6 \\
7 \\
8 \\
9 \\
10\end{array}$ & $\begin{array}{r}6.2 \\
6.6 \\
6.7 \\
6.8 \\
7.8 \\
7.4 \\
8.0 \\
18 \\
12 \\
25\end{array}$ & $\begin{array}{r}42 \\
27 \\
18 \\
15 \\
12 \\
8.4 \\
8.1 \\
7.8 \\
7.5 \\
7.3\end{array}$ & $\begin{array}{r}6.2 \\
6.6 \\
9.3 \\
66 \\
34 \\
28 \\
14 \\
9.7 \\
787 \\
1,540\end{array}$ & $\begin{array}{l}11 \\
12 \\
13 \\
14 \\
15 \\
16 \\
17 \\
18 \\
19 \\
20\end{array}$ & $\begin{array}{c}16 \\
9.5 \\
8.7 \\
9.1 \\
10 \\
11 \\
10 \\
18 \\
37 \\
52\end{array}$ & $\begin{array}{r}7.0 \\
6.7 \\
6.4 \\
6.0 \\
6.4 \\
6.6 \\
7.6 \\
8.7 \\
8.5 \\
35\end{array}$ & $\begin{array}{c}452 \\
42 \\
63 \\
66 \\
24 \\
13 \\
9.3 \\
5.8 \\
4.8 \\
3.8\end{array}$ & $\begin{array}{l}21 \\
22 \\
23 \\
24 \\
25 \\
26 \\
27 \\
28 \\
29 \\
30 \\
31\end{array}$ & \begin{tabular}{r|}
25 \\
29 \\
30 \\
35 \\
21 \\
16 \\
14 \\
12 \\
130 \\
148 \\
61
\end{tabular} & $\begin{array}{c}202 \\
80 \\
29 \\
18 \\
12 \\
9.1 \\
7.6 \\
8.2 \\
6.8 \\
6.4 \\
-\end{array}$ & $\begin{array}{c}4.0 \\
13 \\
102 \\
382 \\
70 \\
42 \\
210 \\
68 \\
19 \\
8.0 \\
4.6\end{array}$ \\
\hline \multicolumn{9}{|c|}{$\begin{array}{l}\text { Monthly mean discharge, in cfs } \ldots \ldots \ldots \ldots \ldots \ldots \ldots \ldots \ldots \ldots \ldots \ldots \ldots \ldots \ldots \ldots \\
\text { Runoff, in acre-feet. } \ldots \ldots \ldots \ldots \ldots \ldots \ldots \ldots\end{array}$} & $\begin{array}{r}26.0 \\
1,600\end{array}$ & $\begin{array}{r}21.0 \\
1,250\end{array}$ & $\begin{array}{r}13 \overline{2} \\
8,150\end{array}$ \\
\hline
\end{tabular}

Gage height, in feet, and discharge, in cubic feet per second, at indicated time, 1950

\begin{tabular}{|c|c|c|c|c|c|c|c|c|c|c|c|}
\hline Hour & $\begin{array}{c}\text { Gage } \\
\text { height }\end{array}$ & Discharge & Hour & $\begin{array}{c}\text { Gage } \\
\text { height }\end{array}$ & Discharge & Hour & $\begin{array}{c}\text { Gage } \\
\text { height }\end{array}$ & Discharge & Hour & $\begin{array}{c}\text { Gage } \\
\text { height }\end{array}$ & Discharge \\
\hline & \multicolumn{2}{|c|}{ July 8} & & \multicolumn{2}{|c|}{ July 9 (con.) } & & \multicolumn{2}{|c|}{ July 11 (con.) } & & \multicolumn{2}{|c|}{ July 13 (con.) } \\
\hline $8 p$ & 2.83 & 7.4 & 9 & 15.06 & 1,280 & 9 & 11.15 & 441 & 11 & 4.06 & 21 \\
\hline $9^{2}$ & 2.82 & 7.3 & 12 & 15.10 & 1,300 & $\mathrm{~N}$ & 8.67 & 244 & $\mathrm{~N}$ & 4.34 & 29 \\
\hline 10 & 3.28 & 16 & & Ju & ly 10 & 3 & 6.67 & 147 & 3 & 5.68 & 98 \\
\hline 11 & 3.80 & 33 & 3 & 15.19 & 1,340 & 6 & 5.82 & 106 & 6 & 6.06 & 121 \\
\hline 12 & 4.45 & 64 & 6 & i5. 3 & 1,440 & 9 & 5.46 & 85 & 9 & 6.12 & 124 \\
\hline & Jul & & 9 & 15.5 & 1,550 & 12 & 5.22 & 70 & 12 & 5.95 & 114 \\
\hline 2 & 6.25 & 172 & $\mathrm{~N}$ & 15.67 & 1,620 & & \multicolumn{2}{|r|}{ July 12} & & \multicolumn{2}{|c|}{ July 14} \\
\hline 4 & 7. 32 & 247 & 3 & 15. 74 & 1,680 & 6 & 4.85 & 52 & 6 & 5.48 & 86 \\
\hline 6 & 8.79 & 350 & 5 & 15.74 & 1,680 & $\mathbf{N}$ & 4.58 & 39 & $\mathrm{~N}$ & 5.05 & 62 \\
\hline 8 & 11.00 & 525 & 6 & $15.7 \mathrm{~s}$ & 1,670 & 6 & 4. 38 & 31 & 6 & 71 & 45 \\
\hline 10 & 12.68 & 666 & 9 & 15.65 & 1,620 & 12 & 4.20 & 25 & 12 & 4.47 & 35 \\
\hline $\mathrm{N}$ & 13.57 & 793 & 12 & 15.45 & 1,490 & & \multicolumn{2}{|c|}{ July 13} & & \multicolumn{2}{|c|}{ July 15} \\
\hline 2 & 14.32 & 1,020 & & $\mathrm{Ju}$ & 1y 11 & 6 & 4.06 & 21 & $\mathbf{N}$ & 4. & 23 \\
\hline 4 & 14.76 & 1,170 & 3 & 14.95 & 1,130 & 10 & 4.00 & 19 & 12 & 3.92 & 17 \\
\hline 6 & 14.98 & 1, & 6 & & 707 & & & & & & \\
\hline
\end{tabular}


Prairie Creek near Silver Creek, Nebr.

Location. --Lat $41^{\circ} 19^{\prime} 15^{\prime \prime}$, long. $97^{\circ} 41^{\prime} 40^{\prime \prime}$, in NW $\frac{1}{4}$ sec. 29 , T. 16 N., R. 3 W., about $200 \mathrm{ft}$ downstream from bridge on State Highway 39, $1 \frac{1}{2}$ miles northwest of Silver Creek and about 11 miles upstream from mouth.

Drainage area. --406 sq $\mathrm{mi}$.

Gage-height record. --Water-stage recorder graph except 7 p. m. July 17 to 2 p. m. July 19 when no record was obtained.

Discharge record. --Stage-discharge relation defined by current-meter measurements. Shifting-control method used.

Maxima.--May-July 1950: Discharge, $889 \mathrm{cfs} 12 \mathrm{~m}$. July 13 (gage height, $6.62 \mathrm{ft}$ ).

August 1949 to April 1950: Díscharge, 127 cfs Sept. 12, 1949 (gage height, $3.03 \mathrm{ft}$ ).

Mean discharge, in cubic feet per second, 1950

\begin{tabular}{|c|c|c|c|c|c|c|c|c|c|c|c|}
\hline Day & May & June & July & Day & May & June & July & Day & May & June & July \\
\hline $\begin{array}{l}1 \\
2 \\
3\end{array}$ & $\begin{array}{l}17 \\
17 \\
17\end{array}$ & $\begin{array}{l}28 \\
30 \\
20\end{array}$ & $\begin{array}{l}13 \\
13 \\
13\end{array}$ & $\begin{array}{l}11 \\
12 \\
13\end{array}$ & $\begin{array}{l}56 \\
78 \\
57\end{array}$ & $\begin{array}{l}11 \\
11 \\
9\end{array}$ & $\begin{array}{l}104 \\
353 \\
862\end{array}$ & $\begin{array}{l}21 \\
22 \\
23\end{array}$ & $\begin{array}{l}23 \\
22 \\
60\end{array}$ & $\begin{array}{l}46 \\
42 \\
51\end{array}$ & $\begin{array}{r}116 \\
100 \\
98\end{array}$ \\
\hline $\begin{array}{r}4 \\
5 \\
6 \\
7 \\
8 \\
9 \\
10\end{array}$ & $\begin{array}{l}17 \\
18 \\
17 \\
17 \\
22 \\
42 \\
38\end{array}$ & $\begin{array}{l}23 \\
23 \\
21 \\
16 \\
13 \\
12 \\
11\end{array}$ & $\begin{array}{r}19 \\
18 \\
16 \\
15 \\
14 \\
50 \\
109\end{array}$ & $\begin{array}{l}14 \\
15 \\
16 \\
17 \\
18 \\
19 \\
20\end{array}$ & $\begin{array}{l}42 \\
34 \\
28 \\
25 \\
23 \\
27 \\
25\end{array}$ & $\begin{array}{c}10 \\
9.4 \\
9.6 \\
24 \\
54 \\
90 \\
58\end{array}$ & $\begin{array}{l}810 \\
752 \\
512 \\
285 \\
180 \\
200 \\
161\end{array}$ & $\begin{array}{l}24 \\
25 \\
26 \\
27 \\
28 \\
29 \\
30 \\
31\end{array}$ & $\begin{array}{r}175 \\
84 \\
52 \\
41 \\
37 \\
35 \\
32 \\
28\end{array}$ & $\begin{array}{l}55 \\
33 \\
22 \\
18 \\
16 \\
15 \\
14 \\
-\end{array}$ & $\begin{array}{r}126 \\
167 \\
228 \\
252 \\
229 \\
170 \\
122 \\
94\end{array}$ \\
\hline \multicolumn{9}{|c|}{$\begin{array}{l}\text { Monthly mean discharge, in cfs } \ldots \ldots \ldots \ldots \ldots \ldots \ldots \ldots \ldots \\
\text { Runoff, in acre-feet. } \ldots \ldots \ldots \ldots \ldots \ldots\end{array}$} & $\begin{array}{r}38.9 \\
2,390\end{array}$ & $\begin{array}{r}26.7 \\
1,590\end{array}$ & $\begin{array}{r}200 \\
12,300\end{array}$ \\
\hline
\end{tabular}

Gage height, in feet, and discharge, in cubic feet per second, at indicated time, 1950

\begin{tabular}{|c|c|c|c|c|c|c|c|c|c|c|c|}
\hline Hour & $\begin{array}{c}\text { Gage } \\
\text { height }\end{array}$ & Discharge & Hour & $\begin{array}{c}\text { Gage } \\
\text { height }\end{array}$ & Discharge & Hour & $\begin{array}{c}\text { Gage } \\
\text { height }\end{array}$ & Discharge & Hour & $\begin{array}{c}\text { Gage } \\
\text { height }\end{array}$ & Discharge \\
\hline & \multicolumn{2}{|c|}{ July 8} & & \multicolumn{2}{|c|}{ July 10} & & \multicolumn{2}{|r|}{ July 12} & & \multicolumn{2}{|r|}{ July 14} \\
\hline $8 p$ & 0.72 & 13 & 4 & 3.00 & 112 & 6 & 3. 51 & 154 & $N$ & 6.49 & 804 \\
\hline 10 & .76 & 14 & 8 & 3.08 & 118 & 9 & 3.77 & 177 & 6 & 6.46 & 787 \\
\hline \multirow[t]{2}{*}{12} & 1.00 & 20 & $\mathrm{~N}$ & 3.01 & 113 & $\mathbf{N}$ & 4. 30 & 229 & & \multicolumn{2}{|c|}{ July 15} \\
\hline & \multicolumn{2}{|c|}{ July 9} & 6 & 2.91 & 106 & 3 & 5.20 & 358 & $\mathrm{~N}$ & 6.42 & 764 \\
\hline 1 & 1.13 & 23 & 12 & 2.81 & 100 & 6 & 6.08 & 603 & 6 & 6.38 & 742 \\
\hline 3 & 1.19 & 25 & & \multicolumn{2}{|c|}{ July 11} & 9 & 6. 38 & 742 & 12 & 6.27 & 686 \\
\hline 6 & 1.34 & 30 & 6 & 2.74 & 96 & 12 & 6.50 & 810 & & \multicolumn{2}{|c|}{ July 16} \\
\hline $\mathrm{N}$ & 1.73 & 46 & $\mathbf{N}$ & 2.81 & 100 & & \multicolumn{2}{|c|}{ July 13} & $\mathbf{N}$ & 5.83 & 515 \\
\hline 6 & 2.18 & 66 & 6 & 2.96 & 109 & 3 & 6.54 & 836 & 12 & 5.32 & 382 \\
\hline 12 & 2.76 & 97 & 12 & 3.22 & 128 & 6 & 6. 56 & 849 & & \multicolumn{2}{|c|}{ July 17} \\
\hline & & & & & & $\mathbf{N}$ & 6.62 & 889 & $\mathbf{N}$ & 4.79 & 290 \\
\hline & & & & & & 6 & 6.60 & 875 & 12 & 4.13 & 211 \\
\hline & & & & & & 12 & 6. 56 & 849 & & & \\
\hline
\end{tabular}


Middle Loup River at St. Paul, Nebr.

Location, --Lat $41^{\circ} 11^{\prime} 55^{\prime \prime}$, long. $98^{\circ} 26^{\prime} 50^{\prime \prime}$, in sec. 10, T, 14 N., R. 10 W., at St. Paul, $600 \mathrm{ft}$ upstream from bridge on U. S. Highway 281 and 6 miles upstream from confluence with North Loup River. Datum of gage is $1,776,6 \mathrm{ft}$ above mean sea level, datum of 1929 .

Drainage area. $-7,720 \mathrm{sq} \mathrm{mi}$, of which only $3,200 \mathrm{sq} \mathrm{mi}$ contribute directly to surface runoff.

Gage-height record. --Water-stage recorder graph except for period June 8-11 when no record was obtained. Record doubtful May 28, June 4-6, because of faulty intake operation.

Discharge record. --Stage-discharge relation defined by current-meter measurements. Shiftingcontrol method used.

Maxima. --May-July 1950: Discharge, 9,600 cfs 11:30 a. m. July 9 (gage height, $6.51 \mathrm{ft}$ ).

1895-97, 1899, 1903, 1928 to April 1950: Discharge, 72, 000 cfs June 23, 1947 (gage height, 10. $69 \mathrm{ft}$ ).

Remarks. - - Diversions above station for irrigation.

Mean discharge, in cubic feet per second, 1950

\begin{tabular}{|c|c|c|c|c|c|c|c|c|c|c|c|}
\hline Day & May & June & July & Day & May & June & July & Day & May & June & July \\
\hline $\begin{array}{r}1 \\
2 \\
3 \\
4 \\
5 \\
6 \\
7 \\
8 \\
9 \\
10\end{array}$ & $\begin{array}{l}1,230 \\
1,070 \\
1,160 \\
1,140 \\
1,450 \\
1,530 \\
1,460 \\
1,450 \\
1,810 \\
1,700\end{array}$ & $\begin{array}{r}1,010 \\
1,020 \\
995 \\
956 \\
870 \\
799 \\
744 \\
740 \\
730 \\
700\end{array}$ & $\begin{array}{r}670 \\
700 \\
788 \\
788 \\
846 \\
918 \\
834 \\
810 \\
6,510 \\
4,830\end{array}$ & $\begin{array}{l}11 \\
12 \\
13 \\
14 \\
15 \\
16 \\
17 \\
18 \\
19 \\
20\end{array}$ & $\begin{array}{l}1,240 \\
1,120 \\
1,300 \\
1,260 \\
1,170 \\
1,220 \\
1,160 \\
1,100 \\
3,920 \\
2,940\end{array}$ & $\begin{array}{r}680 \\
650 \\
630 \\
1,190 \\
1,010 \\
1,010 \\
1,320 \\
3,120 \\
1,900 \\
1,230\end{array}$ & $\begin{array}{r}2,480 \\
2,660 \\
1,500 \\
1,130 \\
969 \\
1,260 \\
1,200 \\
1,430 \\
1,810 \\
1,700\end{array}$ & $\begin{array}{l}21 \\
22 \\
23 \\
24 \\
25 \\
26 \\
27 \\
28 \\
29 \\
30 \\
31\end{array}$ & $\begin{array}{l}2,440 \\
1,810 \\
1,290 \\
1,020 \\
1,090 \\
1,090 \\
1,220 \\
1,540 \\
2,010 \\
1,460 \\
1,130\end{array}$ & $\begin{array}{l}918 \\
810 \\
777 \\
766 \\
700 \\
640 \\
640 \\
755 \\
722 \\
680 \\
-\end{array}$ & $\begin{array}{r}1,510 \\
2,130 \\
4,230 \\
5,380 \\
2,120 \\
1,350 \\
1,230 \\
1 \\
1930 \\
788 \\
969 \\
930\end{array}$ \\
\hline \multicolumn{9}{|c|}{$\begin{array}{l}\text { Monthly mean discharge, in cfs } \ldots \ldots \ldots \ldots \ldots \ldots \ldots \ldots \ldots \ldots \ldots \ldots \ldots \ldots \ldots \\
\text { Runoff, in acre-feet. } \ldots \ldots \ldots \ldots \ldots \ldots \ldots \ldots \ldots\end{array}$} & $\begin{array}{r}1,501 \\
92,290\end{array}$ & $\begin{array}{r}957 \\
56,950\end{array}$ & $\begin{array}{r}1,787 \\
109,900\end{array}$ \\
\hline
\end{tabular}

Gage height, in feet, and discharge, in cubic feet per second, at indicated time, 1950

\begin{tabular}{|c|c|c|c|c|c|c|c|c|c|c|c|}
\hline Hour & $\begin{array}{c}\text { Gage } \\
\text { height }\end{array}$ & Discharge & Hour & $\begin{array}{c}\text { Gage } \\
\text { height }\end{array}$ & Discharge & Hour & $\begin{array}{c}\text { Gage } \\
\text { height }\end{array}$ & Discharge & Hour & $\begin{array}{c}\text { Gage } \\
\text { height }\end{array}$ & Discharge \\
\hline & \multicolumn{2}{|c|}{ July 8} & & \multicolumn{2}{|c|}{ July 9 (con.) } & & \multicolumn{2}{|c|}{ July 10 (con.) } & & \multicolumn{2}{|c|}{ July 12} \\
\hline $9 p$ & $4.47^{\circ}$ & 744 & $11: 30$ & 6.51 & 9,600 & 1 & 5.58 & 4,860 & 6 & 4.85 & 2 , \\
\hline $10^{2}$ & 4.77 & 20 & $\mathrm{~N}$ & & & 3 & & & $\mathrm{~N}$ & 4.83 & \\
\hline 11 & 5.02 & & 1 & 6. & 8, & 6 & 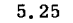 & & 4 & 4.96 & \\
\hline 12 & 5.15 & & 4 & 6. & 8 , & 9 & 5. & & 5 & 4.96 & \\
\hline & Jul & 9 & 5 & 6. & & 12 & 5.04 & 3, & 6 & 4.90 & \\
\hline 1 & 5.40 & 2,910 & 7 & 6. & & & $\mathrm{Ju}$ & y 11 & 7 & 4. & \\
\hline 2 & 60 & & 9 & 6. & & 6 & 4.86 & 2, & 8 & 5. & \\
\hline 3 & 5.56 & 3,630 & 11 & 6.25 & & $\mathrm{~N}$ & 4.64 & 2,130 & $9: 30$ & 5.02 & \\
\hline 5 & 5.50 & 3,450 & & $\mathrm{Ju}$ & $10^{\circ}$ & 3 & 4.49 & 1,840 & 12 & 4.88 & 0 \\
\hline 7 & 5.58 & 3,690 & 3 & 5.89 & 6,260 & $6: 30$ & 4.93 & 2,750 & & & uly 13 \\
\hline 9 & 7 & 4,1 & 6 & 5. & & $8: 30$ & 4.83 & & 6 & 4.39 & \\
\hline 10 & & 5 , & 9 & 5. & & 10 & 4.93 & & $\mathrm{~N}$ & 4.22 & \\
\hline 11 & 6 & & $N$ & 5. 50 & & $\begin{array}{l}11 \\
12\end{array}$ & $\begin{array}{l}4.94 \\
4.88\end{array}$ & $\begin{array}{l}2,770 \\
2,640\end{array}$ & $12 p$ & 4. 18 & \\
\hline
\end{tabular}


Loup River near Genoa, Nebr.

Location. --Lat $41^{\circ} 25^{\prime} 05^{\prime \prime}$, long. $97^{\circ} 43^{\prime} 25^{\prime \prime}$, in NW $\frac{1}{4}$ sec. 25, T. 17 N., R, 4 W., 2 miles south of Genoa, 3 rniles upstream from Beaver Creek, and 6 miles downstream from diversion dam of Loup River Public Power District.

Drainage area. - $-14,440 \mathrm{sq} \mathrm{mi}$, of which only about $6,000 \mathrm{sq} \mathrm{mi}$ contribute directly to surface runoff.

Gage-height record. --Water-stage recorder graph. Record doubtful May 10-18, May 20 to June 1, because of faulty intake operation.

Discharge record. --Stage-discharge relation defined by current-meter measurements except July 11 , $13-18,23-25$, when control was extremely unstable. Shifting-control method used except during period of indefinite stage-discharge relation when discharge was computed on basis of estimated shift adjustments and records for station at Columbus.

Maxima. --May-July 1950: Discharge, $39,300 \mathrm{cfs}$, at $12 \mathrm{~m}$. July $\vartheta$ (gage height, $7.92 \mathrm{ft}$ ).

1928-32, 1943 to A pril 1950: Discharge, 90,000 cfs June 23, 1947 (gage height, $10.12 \mathrm{ft}$ ), from rating curve extended above 32,000 cfs by logarithmic plotting.

Remarks. --Natural flow of stream affected by power developments, ground-water withdrawals and diversions for irrigation, and return flow from irrigated areas.

Records do not include flow of Loup River power canal which diverts at point 6 miles upstream and returns to Platte River below station (see p. 387).

Mean discharge, in cubic feet per second, 1950

\begin{tabular}{|c|c|c|c|c|c|c|c|c|c|c|c|}
\hline Day & May & June & July & Day & May & June & July & Day & May & June & July \\
\hline $\begin{array}{r}1 \\
2 \\
3 \\
4 \\
5 \\
6 \\
7 \\
8 \\
9 \\
10\end{array}$ & $\begin{array}{r}136 \\
91 \\
89 \\
107 \\
204 \\
466 \\
230 \\
443 \\
2,930 \\
1,050\end{array}$ & $\begin{array}{r}179 \\
1,360 \\
1,420 \\
455 \\
295 \\
198 \\
165 \\
176 \\
171 \\
154\end{array}$ & $\begin{array}{r}79 \\
57 \\
100 \\
116 \\
67 \\
70 \\
74 \\
68 \\
25,600 \\
14,000\end{array}$ & $\begin{array}{l}11 \\
12 \\
13 \\
14 \\
15 \\
16 \\
17 \\
18 \\
19 \\
20\end{array}$ & $\begin{array}{r}777 \\
495 \\
443 \\
421 \\
303 \\
234 \\
185 \\
191 \\
1,760 \\
2,010\end{array}$ & $\begin{array}{r}111 \\
103 \\
134 \\
141 \\
179 \\
162 \\
171 \\
361 \\
1,240 \\
390\end{array}$ & $\begin{array}{r}3,750 \\
20,400 \\
4,500 \\
1,030 \\
560 \\
420 \\
6,300 \\
7,000 \\
19,400 \\
3,190\end{array}$ & $\begin{array}{l}21 \\
22 \\
23 \\
24 \\
25 \\
26 \\
27 \\
28 \\
29 \\
30 \\
31\end{array}$ & $\begin{array}{l}897 \\
647 \\
421 \\
274 \\
234 \\
255 \\
234 \\
244 \\
365 \\
654 \\
303 \\
\end{array}$ & $\begin{array}{r}173 \\
136 \\
118 \\
109 \\
70 \\
85 \\
120 \\
128 \\
118 \\
118 \\
-\end{array}$ & $\begin{array}{r}1,560 \\
1,500 \\
10,000 \\
11,500 \\
4,400 \\
2,000 \\
1,560 \\
962 \\
835 \\
421 \\
282\end{array}$ \\
\hline \multicolumn{9}{|c|}{ 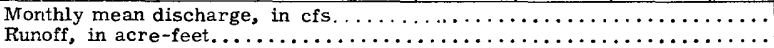 } & $\begin{array}{r}551 \\
33,900\end{array}$ & $\begin{array}{r}291 \\
17,340\end{array}$ & $\begin{array}{r}4,574 \\
281,300\end{array}$ \\
\hline
\end{tabular}

Gage height, in feet, and discharge, in cubic feet per second, at indicated time, 1950

\begin{tabular}{|c|c|c|c|c|c|c|c|c|c|c|c|}
\hline Hour & $\begin{array}{c}\text { Gage } \\
\text { height }\end{array}$ & Discharge & Hour & $\begin{array}{c}\text { Gage } \\
\text { height }\end{array}$ & Discharge & Hour & $\begin{array}{c}\text { Gage } \\
\text { height }\end{array}$ & Discharge & Hour & $\begin{array}{l}\text { Gage } \\
\text { height }\end{array}$ & Discharge \\
\hline & \multicolumn{2}{|c|}{ July 9} & & \multicolumn{2}{|c|}{ July 10 (con) } & & \multicolumn{2}{|c|}{ July 14} & & \multicolumn{2}{|c|}{ July 18 (con.) } \\
\hline $1 \mathrm{a}$ & 2.39 & 154 & 4 & 4.50 & 8,660 & $6 a$ & 2.69 & 1,220 & 4 & 4.67 & 9,120 \\
\hline 2 & 2.47 & 176 & 6 & 4,24 & 7,550 & 2 & 2.29 & 827 & 6 & 5.38 & 13 \\
\hline 3 & 5.30 & 7,510 & 8 & 4.19 & 7,350 & 10 & 2.27 & 810 & 8 & 5.78 & 16,300 \\
\hline 4 & 5.72 & 10,600 & 10 & 4.19 & 7,350 & & & ly 15 & 10 & 5. 68 & 15,600 \\
\hline 5 & 5.88 & 11,800 & 12 & 4.07 & 6,900 & 4 & 2.23 & 625 & 12 & 5.95 & 17,600 \\
\hline 6 & 6.38 & 16,700 & & $\mathrm{Ju}$ & ly 11 & $\mathrm{~N}$ & 2.02 & 432 & & & ruly 19 \\
\hline 7 & 6.60 & 19,500 & 4 & 3.78 & 5,040 & 8 & 1.88 & 316 & 2 & 6.41 & 22,700 \\
\hline 8 & 7.00 & 24,200 & 8 & 3. 39 & 3, & & & 16 & 4 & 6. & 200 \\
\hline 9 & 7. 28 & 27,900 & $\mathrm{~N}$ & 3. & 2,600 & 4 & 1.82 & 290 & 6 & 6. & 25,100 \\
\hline 10 & 7.45 & 30,800 & 4 & 2. & 1,8 & $\mathrm{~N}$ & 1.68 & 237 & $\mathbf{N}$ & & 23,100 \\
\hline 11 & 7.70 & 35,000 & 8 & 2. & 1, & 5 & 1.65 & 227 & 4 & & 20,200 \\
\hline $\mathrm{N}$ & 7.92 & 39,300 & 10 & 2.6 & 1,720 & 7 & 1.70 & 244 & 6 & 5. & 16,000 \\
\hline 1 & 7.88 & 38,900 & 12 & 5.78 & 17,100 & 8 & 1.80 & 316 & 8 & 4.96 & 10,600 \\
\hline 3 & 7.79 & 37,600 & & Ju & ly 12 & 10 & 1.96 & 352 & 10 & 4.45 & 7,59 \\
\hline 5 & 7.62 & 35,400 & 2 & 6.90 & 28 , & 12 & 3.46 & 3,600 & 12 & 4.05 & 5,830 \\
\hline 7 & 7.44 & 33,200 & 4 & 7.19 & 31 & & & ly 17 & & & ruly 20 \\
\hline 9 & 7.28 & 31,200 & 6 & 6.96 & 29,000 & 2 & 4.62 & 8,010 & 4 & 3.6 & 4,150 \\
\hline 11 & 7.13 & 29,400 & 8 & 6. & 24,700 & 4 & 4. 84 & 9,960 & 8 & & 3,480 \\
\hline & & 1010 & 10 & 6.19 & 21,700 & 6 & 4.92 & 10,400 & $\mathbf{N}$ & & 2,780 \\
\hline 2 & 6.85 & 26,300 & $\mathrm{~N}$ & 6.03 & 20,400 & 8 & 4.72 & 9,370 & 4 & & 2,470 \\
\hline 4 & 6.49 & 22,700 & 4 & 5. & & $\mathrm{~N}$ & 4.19 & & 8 & & \\
\hline 6 & 6.09 & 19,100 & 8 & 4.94 & 12 , & 6 & 3.47 & 3,630 & 12 & 2.88 & 2,200 \\
\hline 8 & 5.75 & 16,400 & 12 & 4.28 & 9,080 & 12 & 3.12 & 2,450 & & & ruly 21 \\
\hline 10 & 5.38 & 13,700 & & $\mathrm{Ju}$ & ly 13 & & & ly 18 & $\mathbf{N}$ & 2.45 & 1,440 \\
\hline $\mathrm{N}$ & 5.03 & 11,500 & 6 & 3.89 & 5,3 & 4 & 3.02 & 2,270 & & & \\
\hline 2 & 4.75 & 9,860 & 10 & 3.87 & 5,320 & 10 & 2.98 & 2,200 & & & \\
\hline & & & $\begin{array}{l}2 \\
6\end{array}$ & $\begin{array}{l}3.39 \\
3.26\end{array}$ & $\begin{array}{l}3,440 \\
3,140\end{array}$ & $\mathrm{~N}_{2}$ & $\begin{array}{l}3.30 \\
3.95\end{array}$ & $\begin{array}{l}2,800 \\
4,950\end{array}$ & & & \\
\hline
\end{tabular}


Loup River at Columbus, Nebr.

Location. --Lat $41^{\circ} 24^{\prime} 50^{\prime \prime}$, long. $97^{\circ} 22^{\prime} 00^{\prime \prime}$, in sec. 30, T. $17 \mathrm{~N}$., R. $1 \mathrm{E}$, , at bridge on U. S. Highway 30 at Columbus, $3 \frac{1}{2}$ miles upstream from mouth and 14 miles downstream from Lookinglass Creek. Datum of gage is 1, 430, $29 \mathrm{ft}$ above mean sea level, unadjusted. Prior to Aug. 28, 1950 at datum 2.00 ft higher. Gage heights contained herein are to present datum.

Drainage area. $--15,230 \mathrm{sq} \mathrm{mi}$, of which only about $6,530 \mathrm{sq} \mathrm{mi}$ contribute directly to surface runoff.

Gage-height record. --Wire-weight gage read twice daily except July 4. Mean daily gage heights May 9, $10,19,20$, June 2,3 , July 9-14, 17-25, computed from graphs based on gage readings.

Discharge record. --Stage-discharge relation defined by current-meter measurements below 30,000 cfs and extended upward by logarithmic ploting. Shifting-control method used. Discharge for July 4 computed on basis of records for station near Genoa.

Maxima.--May-July 1950: Discharge, 42, $100 \mathrm{cfs} 2$ a. m. July 10 (gage height, 9.0 ft).

1894-1915, 1931, 1933 to April 1950: Discharge, 85, $000 \mathrm{cfs}$ June 23, 1947 (gage height, $12.0 \mathrm{ft}$, from floodmark), from rating curve extended above 30,000 cfs by logarithmic plotting.

Remarks. - Natural flow of stream affected by power developments, ground water withdrawals and diversions for irrigation, and return flow from irrigated areas. Records do not include flow of Loup River power canal which diverts at point 25 miles upstream and returns to Platte River below station. (See p. 387).

Mean discharge, in cubic feet per second, 1950

\begin{tabular}{|c|c|c|c|c|c|c|c|c|c|c|c|}
\hline Day & May & June & July & Day & May & June & July & Day & May & June & $\overline{\text { July }}$ \\
\hline $\begin{array}{r}1 \\
2 \\
3 \\
4 \\
5 \\
6 \\
7 \\
8 \\
9 \\
10\end{array}$ & $\begin{array}{r}356 \\
302 \\
320 \\
342 \\
423 \\
512 \\
463 \\
487 \\
4,740 \\
2,060\end{array}$ & $\begin{array}{r}406 \\
2,820 \\
4,600 \\
2,230 \\
1,540 \\
1,260 \\
970 \\
655 \\
524 \\
376\end{array}$ & $\begin{array}{r}262 \\
156 \\
173 \\
180 \\
193 \\
184 \\
178 \\
206 \\
17,400 \\
24,100\end{array}$ & $\begin{array}{l}11 \\
12 \\
13 \\
14 \\
15 \\
16 \\
17 \\
18 \\
19 \\
20\end{array}$ & $\begin{array}{r}1,290 \\
889 \\
662 \\
708 \\
531 \\
445 \\
385 \\
324 \\
1,320 \\
3,410\end{array}$ & $\begin{array}{r}333 \\
258 \\
254 \\
298 \\
294 \\
356 \\
356 \\
469 \\
2,160 \\
863\end{array}$ & $\begin{array}{r}7,090 \\
18,800 \\
9,880 \\
2,030 \\
538 \\
311 \\
5,580 \\
6,600 \\
24,800 \\
8,970\end{array}$ & $\begin{array}{l}21 \\
22 \\
23 \\
24 \\
25 \\
26 \\
27 \\
28 \\
29 \\
30 \\
31\end{array}$ & $\begin{array}{r}1,260 \\
916 \\
\mathbf{7 6 3} \\
\mathbf{5 9 1} \\
418 \\
\mathbf{5 2 4} \\
\mathbf{5 1 2} \\
\mathbf{4 8 1} \\
\mathbf{7 0 8} \\
1,190 \\
\mathbf{7 5 5}\end{array}$ & $\begin{array}{l}493 \\
352 \\
311 \\
258 \\
254 \\
196 \\
206 \\
187 \\
181 \\
196 \\
-\end{array}$ & $\begin{array}{r}3,500 \\
3,210 \\
10,80 C \\
14,20 C \\
4,70 C \\
2,30 C \\
2,42 C \\
1,69 c \\
1,27 C \\
863 \\
70 C\end{array}$ \\
\hline \multicolumn{12}{|c|}{ 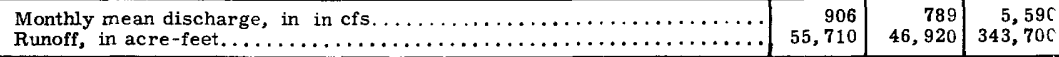 } \\
\hline
\end{tabular}

Gage height, in feet, and discharge, in cubic feet per second, at indicated time, 1950

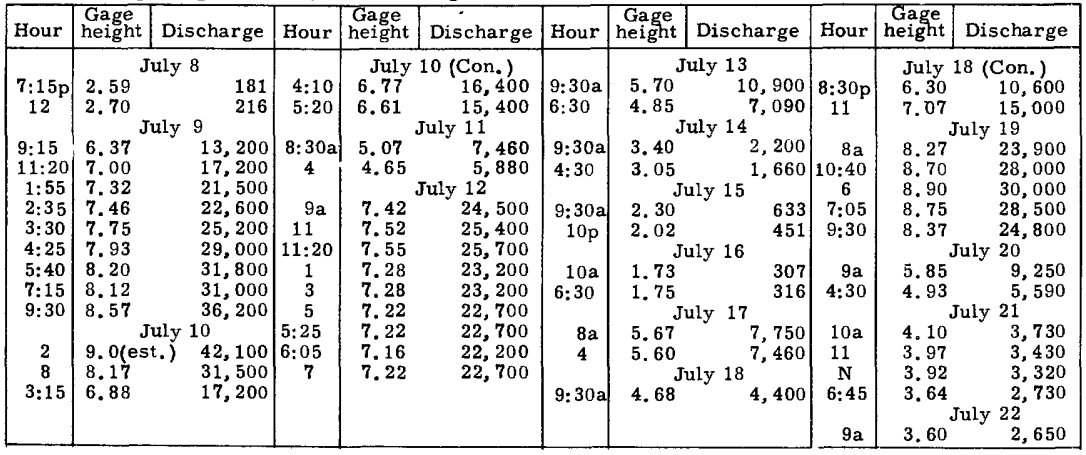


North Loup River near St. Paul, Nebr.

Location. --Lat $41^{\circ} 15^{\prime} 35^{\prime \prime}$, long. $98^{\circ} 26^{\prime} 50^{\prime \prime}$, in sec. $22, \mathrm{~T}, 15 \mathrm{~N}$., R. $10 \mathrm{~W} ., 60$ ft downstream from bridge on U. S. Highway 281, 3 miles north of St. Paul, and 4 miles upstream from confluence with Middle Loup River.

Drainage area. $--4,460$ sq $\mathrm{mi}$, of which only about 1,270 sq mi contribute directly to surface runoff.

Gage-height record.--Water-stage recorder graph.

Discharge record. --Stage-discharge relation defined by current-meter measurements. Shiftingcontrol method used.

Maxima. --May-July 1950: Discharge, 16,800 cfs 5:30 a. m. July 9 (gage height, 4, $37 \mathrm{ft}$ ).

1895-97, 1899-1903, 1928 to April 1950: Discharge, 90, 000 cfs (estimated) June 6, 1896.

Remarks. --Natural flow affected by ground water withdrawals and diversions for irrigation, and return flow from irrigated areas.

Mean discharge, in cubic feet per second, 1950

\begin{tabular}{|c|c|c|c|c|c|c|c|c|c|c|c|}
\hline Day & May & June & July & Day & May & June & July & Day & May & June & July \\
\hline $\begin{array}{r}1 \\
2 \\
3 \\
4 \\
5 \\
6 \\
7 \\
8 \\
9 \\
10\end{array}$ & $\begin{array}{r}920 \\
946 \\
985 \\
1,010 \\
1,140 \\
1,280 \\
1,330 \\
1,660 \\
1,780 \\
1,460\end{array}$ & $\begin{array}{l}920 \\
874 \\
920 \\
851 \\
874 \\
886 \\
816 \\
732 \\
672 \\
614\end{array}$ & $\begin{array}{r}533 \\
574 \\
824 \\
662 \\
710 \\
784 \\
721 \\
837 \\
10,200 \\
2,350\end{array}$ & $\begin{array}{l}11 \\
12 \\
13 \\
14 \\
15 \\
16 \\
17 \\
18 \\
19 \\
20\end{array}$ & $\begin{array}{l}1,360 \\
1,280 \\
1,390 \\
1,250 \\
1,120 \\
1,060 \\
1,040 \\
1,020 \\
2,270 \\
1,340\end{array}$ & $\begin{array}{l}\mathbf{5 9 6} \\
\mathbf{5 7 8} \\
\mathbf{5 6 9} \\
\mathbf{7 5 2} \\
\mathbf{7 9 4} \\
\mathbf{7 4 2} \\
\mathbf{9 7 2} \\
\mathbf{9 7 2} \\
998 \\
\mathbf{8 9 7}\end{array}$ & $\begin{array}{r}1,280 \\
1,950 \\
1,010 \\
934 \\
852 \\
1,090 \\
1,230 \\
2,540 \\
3,090 \\
1,480\end{array}$ & $\begin{array}{l}21 \\
22 \\
23 \\
24 \\
25 \\
26 \\
27 \\
28 \\
29 \\
30 \\
31\end{array}$ & $\begin{array}{r}1,360 \\
1,260 \\
1,170 \\
1,040 \\
946 \\
985 \\
998 \\
1,140 \\
1,220 \\
1,170 \\
998\end{array}$ & $\begin{array}{l}851 \\
794 \\
763 \\
681 \\
605 \\
560 \\
560 \\
551 \\
551 \\
551 \\
-\end{array}$ & $\begin{array}{r}1,270 \\
1,270 \\
6,590 \\
2,570 \\
1,210 \\
1,060 \\
962 \\
915 \\
852 \\
817 \\
769\end{array}$ \\
\hline \multicolumn{9}{|c|}{$\begin{array}{l}\text { Monthly mean discharge, in cfs. } \\
\text { Runoff, in acre-feet........... }\end{array}$} & $\begin{array}{r}1,223 \\
75,230\end{array}$ & $\begin{array}{r}750 \\
44,620\end{array}$ & $\begin{array}{r}1,675 \\
103,000\end{array}$ \\
\hline
\end{tabular}

Gage height, in feet, and discharge, in cubic feet per second, at indicated time, 1950

\begin{tabular}{|c|c|c|c|c|c|c|c|c|c|c|c|}
\hline Hour & $\begin{array}{l}\text { Gage } \\
\text { height }\end{array}$ & Discharge & Hour & $\begin{array}{c}\text { Gage } \\
\text { height }\end{array}$ & Discharge & Hour & $\begin{array}{c}\text { Gage } \\
\text { height }\end{array}$ & Discharge & Hour & $\begin{array}{c}\text { Gage } \\
\text { height }\end{array}$ & Discharge \\
\hline & \multicolumn{2}{|c|}{ July 8} & \multirow{7}{*}{$\begin{array}{r}10 \\
\mathrm{~N} \\
4 \\
8 \\
12\end{array}$} & \multicolumn{2}{|c|}{ July 9 (Con,) } & \multirow{10}{*}{$\begin{array}{c}6 \\
N \\
3 \\
5 \\
6: 30 \\
7 \\
9: 30 \\
12\end{array}$} & \multicolumn{2}{|c|}{ July 11} & & \multicolumn{2}{|c|}{ July 12 (Con.) } \\
\hline $8 p$ & 1.07 & 690 & & 3.67 & 13,300 & & $0.90^{\circ}$ & 1,230 & 4 & 2.16 & 3,790 \\
\hline 9 & 1.10 & 721 & & 3.47 & 11,800 & & .80 & 1,120 & $4: 30$ & 2.18 & 3,850 \\
\hline 10 & 1.50 & 1,230 & & 3,05 & 9,000 & & & 1,060 & 5 & 2,16 & 90 \\
\hline 11 & 2.07 & 2,330 & & 2.64 & 6,320 & & .83 & 1,150 & 7 & 1.90 & 140 \\
\hline 12 & 2.85 & 4,630 & & 2.15 & 4,030 & & 1.36 & & 9 & 1.60 & 10 \\
\hline & & y 9 & & & Suly 10 & & 1.20 & & $\mathbf{N}$ & .22 & 50 \\
\hline 1 & 2. 55 & 4,000 & 4 & 1.88 & 2,990 & & 1.05 & 420 & 4 & .99 & 40 \\
\hline 2 & 3.89 & 12,300 & 8 & 1.79 & 2,750 & & 1.26 & 1,710 & 8 & 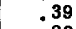 & 0 \\
\hline 3 & 3. 96 & 13,000 & N & 1.65 & 2,420 & & $\mathbf{J}$ & 12 & 12 & .83 & 1 \\
\hline 4 & 4.06 & 13,900 & 4 & 1.42 & 1,970 & $1 \mathbf{a}$ & 1.49 & 2,090 & & 072 & uly 13 \\
\hline $5: 30$ & 4. 37 & 16,800 & 8 & 1. & 1,640 & 2 & 1.59 & 2,290 & 6 & 0.72 & 1,030 \\
\hline $\begin{array}{l}7 \\
8\end{array}$ & $\begin{array}{l}4.23 \\
4.01\end{array}$ & $\begin{array}{l}15,900 \\
14,500\end{array}$ & 12 & & & 3 & 2.01 & & $\begin{array}{r}N \\
12\end{array}$ & $\begin{array}{r}.69 \\
.65\end{array}$ & $\begin{array}{r}1,000 \\
962\end{array}$ \\
\hline
\end{tabular}


Spring Creek at Cushing, Nebr.

Location. --Lat $41^{\circ} 18^{\prime} 00^{\prime \prime}$, long, $98^{\circ} 22^{\prime} 50^{\prime \prime}$, in SW $\frac{1}{4}$ sec. 5, T. $15 \mathrm{~N} .$, R. 9 W., about one-half mile northwest of Cushing and about $2 \frac{1}{2}$ miles upstream from mouth.

Gage-height record. --Water-stage recorder graph except May 9-14, 24, July 21, 25, 26, when no record was obtained because of faulty intake action, and May $8,15,18,19,23,25$, May 28 to June 3 , June $6,14,16-18$, July $9-12,16-20,22,24,27$, when fragmentary record was obtained. Gage heights for days of partial record were obtained from estimated graph.

Discharge record. --Stage-discharge relation defined by current-meter measurements below 1, 500 cfs and extended to peak stage by logarithmic plotting. Discharge for periods of no gage-height record computed on basis of weather records and trend of flow.

Maxima. --May-July 1950: Discharge, 2, $560 \mathrm{cfs} 11: 30$ p. m. July 22 (gage height, 15. $00 \mathrm{ft}$ ).

May 1949 to April 1950: Discharge, 448 cfs June 27, 1949 (gage height, 5. $79 \mathrm{ft}$ ).

Mean discharge, in cubic feet per second, 1950

\begin{tabular}{|c|c|c|c|c|c|c|c|c|c|c|c|}
\hline Day & May & June & July & Day & May & June & July & Day & May & June & July \\
\hline $\begin{array}{r}1 \\
2 \\
3 \\
4 \\
5 \\
6 \\
7 \\
8 \\
9 \\
10\end{array}$ & $\begin{array}{r}8.2 \\
7.6 \\
7.3 \\
7.0 \\
7.3 \\
7.9 \\
7.0 \\
72 \\
35 \\
18\end{array}$ & $\begin{array}{r}7.0 \\
6.3 \\
11 \\
6.6 \\
6.0 \\
5.4 \\
5.7 \\
4.9 \\
4.6 \\
4.4\end{array}$ & $\begin{array}{c}2.8 \\
3.3 \\
6.0 \\
12 \\
10 \\
5.7 \\
5.1 \\
78 \\
331 \\
428\end{array}$ & $\begin{array}{l}11 \\
12 \\
13 \\
14 \\
15 \\
16 \\
17 \\
18 \\
19 \\
20\end{array}$ & $\begin{array}{c}11 \\
9.2 \\
8.1 \\
7.6 \\
7.3 \\
7.3 \\
7.0 \\
46 \\
118 \\
30\end{array}$ & $\begin{array}{c}3.8 \\
3.8 \\
3.6 \\
12 \\
5.1 \\
4.9 \\
27 \\
13 \\
9.2 \\
11\end{array}$ & $\begin{array}{r}127 \\
108 \\
54 \\
17 \\
13 \\
33 \\
231 \\
641 \\
1,080 \\
147\end{array}$ & $\begin{array}{l}21 \\
22 \\
23 \\
24 \\
25 \\
26 \\
27 \\
28 \\
29 \\
30 \\
31\end{array}$ & $\begin{array}{c}16 \\
15 \\
14 \\
12 \\
11 \\
9.6 \\
9.2 \\
9.2 \\
11 \\
10 \\
8.5\end{array}$ & \begin{tabular}{l|}
6.0 \\
4.6 \\
4.1 \\
3.8 \\
3.1 \\
3.1 \\
3.1 \\
3.1 \\
2.8 \\
2.8 \\
-
\end{tabular} & $\begin{array}{r}25 \\
232 \\
365 \\
135 \\
30 \\
17 \\
14 \\
12 \\
11 \\
10 \\
8.5\end{array}$ \\
\hline \multicolumn{9}{|c|}{ 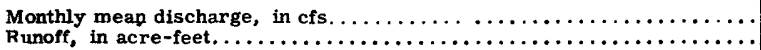 } & $\begin{array}{r}17.9 \\
1,100\end{array}$ & \begin{tabular}{r|}
6.39 \\
380
\end{tabular} & $\begin{array}{r}135 \\
8,320\end{array}$ \\
\hline
\end{tabular}

Gage height, in feet, and discharge, in cubic feet per second, at indicated time, 1950

\begin{tabular}{|c|c|c|c|c|c|c|c|c|c|c|c|}
\hline Hour & $\begin{array}{l}\text { Gage } \\
\text { height }\end{array}$ & Discharge & Hour & $\begin{array}{l}\text { Gage } \\
\text { height }\end{array}$ & Discharge & Hour & $\begin{array}{c}\text { Gage } \\
\text { height }\end{array}$ & Discharge & Hour & $\begin{array}{l}\text { Gage } \\
\text { height }\end{array}$ & Discharge \\
\hline \multirow{17}{*}{$\begin{array}{c}9 \mathrm{p} \\
10 \\
11 \\
12 \\
\\
2 \mathrm{a} \\
4 \\
6 \\
8 \\
10 \\
N \\
\\
5 \mathrm{a} \\
6 \\
7 \\
8 \\
9\end{array}$} & \multicolumn{2}{|c|}{ July 8} & & \multicolumn{2}{|c|}{ July 18 (Con.) } & & \multicolumn{2}{|c|}{ July 19 (Con.) } & \multirow{4}{*}{$\begin{array}{l}4 \\
6 \\
8\end{array}$} & \multirow{2}{*}{\multicolumn{2}{|c|}{ July 23 (Con.) }} \\
\hline & 1.50 & 4.9 & 10 & 8.30 & 877 & 9 & 6.95 & 632 & & & 582 \\
\hline & 3.64 & 156 & 11 & 8.65 & 945 & 12 & 5.85 & 446 & & 4.67 & 274 \\
\hline & 9.50 & 1,120 & $\mathbf{N}$ & 9.20 & 1,060 & & & lly 20 & & 3.77 & 166 \\
\hline & 8.86 & 995 & & 9.38 & 1,090 & $4 a$ & 4.65 & 266 & 10 & 3.31 & 118 \\
\hline & $\mathrm{J}$ & dy 9 & 3 & 9.20 & 1,060 & 8 & 3.78 & 156 & $\mathbf{N}$ & 3.35 & 121 \\
\hline & 6.14 & 507 & 6 & 8.52 & 919 & $\mathbf{N}$ & 3.25 & 100 & 2 & 3.82 & 170 \\
\hline & 5.52 & 413 & 9 & 7.48 & 727 & 4 & 2.87 & 70 & 4 & 4.08 & 198 \\
\hline & 4.67 & 293 & 11 & 7.13 & 663 & 8 & 2.67 & 56 & 6 & 4.19 & 212 \\
\hline & 3.77 & 184 & 12 & 7.47 & 725 & 12 & 2.47 & 42 & 8 & 4.27 & 221 \\
\hline & 3. 54 & 159 & & & July 19 & & $\mathrm{~J}$ & 1 & 10 & 4.47 & 245 \\
\hline & & 147 & $3 \mathbf{a}$ & 9.35 & 1,090 & $9 p$ & 2.36 & 35 & 12 & 4.66 & 268 \\
\hline & $\mathrm{J}$ & aly 18 & B & 10.35 & 1,310 & $10^{\circ}$ & 11.90 & 1,690 & & & July 24 \\
\hline & 2.56 & 77 & 9 & 11.07 & 1,480 & 11 & 14.48 & 2,410 & $\begin{array}{ll}2 \mathrm{a} \\
6\end{array}$ & $\begin{array}{l}4.75 \\
4.40\end{array}$ & $\begin{array}{l}280 \\
237\end{array}$ \\
\hline & 2.56 & $\begin{array}{r}77 \\
214\end{array}$ & 11 & 11.20 & 1,520 & $11: 30$ & 15.00 & 2,560 & 6 & $\begin{array}{l}4.40 \\
3.10\end{array}$ & $\begin{array}{r}237 \\
99\end{array}$ \\
\hline & $\begin{array}{l}3.94 \\
7.00\end{array}$ & $\begin{array}{l}214 \\
649\end{array}$ & $\begin{array}{l}\mathbf{N} \\
\mathbf{3}\end{array}$ & $\begin{array}{r}11.07 \\
9.80\end{array}$ & $\begin{array}{l}1,480 \\
1,190\end{array}$ & 12 & $14.60 \mathrm{~J}$ & ly $23^{2,}$ & $\begin{array}{l}\text { IN } \\
6\end{array}$ & 2.54 & 56 \\
\hline & & 793 & 6 & 8.10 & 839 & $2 \mathbf{a}$ & 7.43 & 717 & 12 & 2.35 & 42 \\
\hline
\end{tabular}


Cedar River near Spalding, Nebr.

Location. --Lat $41^{\circ} 43^{\prime} 20^{\prime \prime}$, long. $98^{\circ} 27^{\prime} 45^{\prime \prime}$, in NW' sec. 5, T. 20 N., R. 10 W, , 40 ft downstream from highway bridge, $10 \frac{1}{2}$ miles northwest of Spalding.

Drainage area. $--794 \mathrm{sq} \mathrm{mi}$, of which only about $50 \mathrm{sq} \mathrm{mi}$ contribute directly to surface runoff.

Gage-height record. --Water-stage recorder graph.

Discharge record. --Stage-discharge relation defined by current-meter measurements below 650 cfs and extended to peak stage on basis of slope-area determination at gage height $7.50 \mathrm{ft}$. Shifting-control method used.

Maxima. --May-July 1950: Discharge, 1,320 cfs, 9:30 p. m. July 8 (gage height, $5.12 \mathrm{ft}$ ).

1945 to April 1950: Discharge, 4, 000 cfs June 23, 1947 (gage height, 7. $50 \mathrm{ft}$ ), by slope-area method.

Remarks. --Low and medium flow regulated by power plant above station.

Mean discharge, in cubic feet per second, 1950

\begin{tabular}{|c|c|c|c|c|c|c|c|c|c|c|c|}
\hline Day & May & June & July & Day & May & June & July & Day & May & June & July \\
\hline $\begin{array}{r}1 \\
2 \\
3 \\
4 \\
5 \\
6 \\
7 \\
8 \\
9 \\
10\end{array}$ & $\begin{array}{l}153 \\
139 \\
162 \\
171 \\
166 \\
166 \\
173 \\
180 \\
278 \\
264\end{array}$ & $\begin{array}{l}161 \\
224 \\
271 \\
230 \\
256 \\
324 \\
327 \\
289 \\
209 \\
168\end{array}$ & $\begin{array}{l}125 \\
124 \\
162 \\
162 \\
166 \\
150 \\
138 \\
265 \\
545 \\
312\end{array}$ & $\begin{array}{l}11 \\
12 \\
13 \\
14 \\
15 \\
16 \\
17 \\
18 \\
19 \\
20\end{array}$ & $\begin{array}{l}209 \\
254 \\
244 \\
207 \\
194 \\
148 \\
153 \\
188 \\
240 \\
277\end{array}$ & $\begin{array}{l}156 \\
157 \\
128 \\
137 \\
170 \\
150 \\
140 \\
173 \\
187 \\
171\end{array}$ & $\begin{array}{l}326 \\
623 \\
628 \\
524 \\
472 \\
532 \\
543 \\
499 \\
521 \\
352\end{array}$ & $\begin{array}{l}21 \\
22 \\
23 \\
24 \\
25 \\
26 \\
27 \\
28 \\
29 \\
30 \\
31 \\
\end{array}$ & $\begin{array}{r}200 \\
272 \\
244 \\
\times 258 \\
208 \\
193 \\
183 \\
179 \\
192 \\
163 \\
174 \\
\end{array}$ & $\begin{array}{r}179 \\
194 \\
206 \\
187 \\
176 \\
148 \\
130 \\
119 \\
94 \\
116 \\
-\quad \\
\end{array}$ & $\begin{array}{l}317 \\
306 \\
388 \\
316 \\
285 \\
264 \\
224 \\
186 \\
259 \\
159 \\
178 \\
\end{array}$ \\
\hline \multicolumn{9}{|c|}{$\begin{array}{l}\text { Monthly mean discharge, cfs. } \ldots \ldots \ldots \ldots \ldots \ldots \ldots \ldots \ldots \ldots \ldots \ldots \ldots \ldots \ldots \ldots \ldots \ldots \\
\text { Runoff, in acre-feet. } \ldots \ldots \ldots \ldots \ldots \ldots \ldots \ldots \ldots \ldots \ldots \ldots \ldots \ldots \ldots \\
\end{array}$} & $\begin{array}{r}201 \\
12,360 \\
\end{array}$ & $\begin{array}{r}186 \\
11,060 \\
\end{array}$ & $\begin{array}{r}324 \\
19,940 \\
\end{array}$ \\
\hline
\end{tabular}

Gage height, in feet, and discharge, in cubic feet per second, at indicated time, 1950

\begin{tabular}{|c|c|c|c|c|c|c|c|c|c|c|c|}
\hline Hour & $\begin{array}{l}\text { Gage } \\
\text { height }\end{array}$ & Discharge & our & $\begin{array}{l}\text { Gage } \\
\text { height }\end{array}$ & Discharge & Hour & $\begin{array}{l}\text { Gage } \\
\text { height }\end{array}$ & Discharge & lour & $\begin{array}{c}\text { Gage } \\
\text { height }\end{array}$ & Discharge \\
\hline $\begin{array}{c}3 \mathrm{a} \\
6 \\
9 \\
12 \\
5\end{array}$ & $\begin{array}{l}2.22 \\
1.87 \\
1.37 \\
1.18 \\
1.10\end{array}$ & $\begin{array}{r}230 \\
162 \\
87 \\
63 \\
54\end{array}$ & $\begin{array}{c}6 \\
7 \\
7: 45 \\
8 \\
9 \\
9: 30\end{array}$ & $\begin{array}{l}\text { July } \\
1.72 \\
2.07 \\
2.13 \\
2.50 \\
4.94 \\
5.12\end{array}$ & \begin{tabular}{r|} 
(con.) \\
137 \\
199 \\
211 \\
294 \\
1,210 \\
1,320
\end{tabular} & $\begin{array}{l}10 \\
11 \\
12 \\
3 a \\
6\end{array}$ & $\begin{array}{l}\text { July } \\
4.90 \\
4.40 \\
4.05 \\
\\
3.73 \\
3.43\end{array}$ & $\begin{array}{rr}\text { (con.) } \\
1,190 \\
934 \\
787 \\
\text { July } 9 \\
\\
634 \\
532\end{array}$ & $\begin{array}{r}10 \\
2 \\
4 \\
6 \\
8 \\
10 \\
12 \\
\end{array}$ & $\begin{array}{l}3.94 \\
3.78 \\
3.20 \\
2.61 \\
2.21 \\
2.45\end{array}$ & \begin{tabular}{|r|}
9 (con.) \\
634 \\
710 \\
651 \\
461 \\
300 \\
209 \\
261 \\
\end{tabular} \\
\hline
\end{tabular}


Cedar River near Fullerton, Nebr.

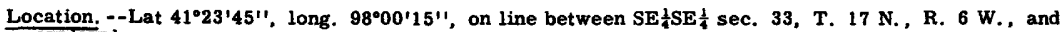
$\mathrm{NE} \frac{1}{4} \mathrm{NE} \frac{1}{4} \mathrm{sec} .4, \mathrm{~T} .16 \mathrm{~N}$., R. 6 W., at bridge on State Highway 52, 3 miles northwest of Fullerton and $53 / 4$ miles upstream from mouth.

Drainage area. $--1,220 \mathrm{sq} \mathrm{mi}$, of which only about $480 \mathrm{sq} \mathrm{mi}$ contribut e directly to surface runoff.

Gage-height record. --Water-stage recorder graph, except periods June 15-26, July 8, when twicedailly staff gage readings were used.

Discharge record. --Stage-discharge relation defined by current-meter measurementa. Shifting-control method used.

Maxima. --May-July 1950: Discharge, 10, 100 cfs 1:30 a. m. July 19 (gage height, 9.64ft).

1931-32, 1940 to April 1950: Discharge, 9, 760 cfs Feb. 28, 1948 (gage height, 9. $40 \mathrm{ft}$ ).

Remarks. --Natural flow affected by power developments, ground-water withdrawals for irrigation, and return flow from irrigated areas.

Mean discharge, in cubic feet per second, 1950

\begin{tabular}{|c|c|c|c|c|c|c|c|c|c|c|c|}
\hline Day & May & June & July & Day & May & June & July & Day & May & June & July \\
\hline $\begin{array}{r}1 \\
2 \\
3 \\
4 \\
5 \\
6 \\
7 \\
8 \\
9 \\
10\end{array}$ & $\begin{array}{r}222 \\
222 \\
228 \\
228 \\
281 \\
287 \\
287 \\
497 \\
1,150 \\
371\end{array}$ & $\begin{array}{r}\mathbf{2 9 0} \\
\mathbf{1} \mathbf{5 7 0} \\
\mathbf{4 8 2} \\
\mathbf{3 3 2} \\
\mathbf{3 2 0} \\
\mathbf{3 3 2} \\
\mathbf{3 3 8} \\
\mathbf{3 9 0} \\
\mathbf{3 8 0} \\
\mathbf{3 2 9}\end{array}$ & $\begin{array}{r}147 \\
164 \\
479 \\
320 \\
250 \\
253 \\
233 \\
378 \\
5,780 \\
1,750\end{array}$ & $\begin{array}{l}11 \\
12 \\
13 \\
14 \\
15 \\
16 \\
17 \\
18 \\
19 \\
20\end{array}$ & $\begin{array}{l}308 \\
317 \\
320 \\
329 \\
296 \\
267 \\
250 \\
228 \\
422 \\
341\end{array}$ & $\begin{array}{l}281 \\
230 \\
230 \\
236 \\
261 \\
258 . \\
244 \\
441 \\
261 \\
276\end{array}$ & $\begin{array}{r}1,870 \\
5,320 \\
1,410 \\
1,150 \\
971 \\
1,620 \\
2,820 \\
3,920 \\
6,430 \\
1,110\end{array}$ & $\begin{array}{l}21 \\
22 \\
23 \\
24 \\
25 \\
26 \\
27 \\
28 \\
29 \\
30 \\
31\end{array}$ & $\begin{array}{l}\mathbf{3 1 7} \\
\mathbf{2 9 9} \\
\mathbf{3 2 9} \\
\mathbf{3 0 8} \\
\mathbf{3 3 2} \\
\mathbf{3 5 3} \\
\mathbf{3 0 2} \\
\mathbf{3 1 1} \\
\mathbf{3 3 5} \\
\mathbf{3 2 3} \\
\mathbf{2 9 6}\end{array}$ & $\begin{array}{l}241 \\
228 \\
217 \\
239 \\
217 \\
214 \\
198 \\
179 \\
173 \\
164 \\
-\end{array}$ & $\begin{array}{l}908 \\
678 \\
895 \\
721 \\
626 \\
558 \\
473 \\
435 \\
386 \\
359 \\
353\end{array}$ \\
\hline Mo & & & & & & & & & $\begin{array}{r}334 \\
20,540\end{array}$ & $\begin{array}{r}318 \\
18,940\end{array}$ & $\begin{array}{r}1,380 \\
84,830\end{array}$ \\
\hline
\end{tabular}

Gage height, in feet, and discharge, in cubic feet per second, at indicated time, 1950

\begin{tabular}{|c|c|c|c|c|c|c|c|c|c|c|c|}
\hline Hour & $\begin{array}{c}\text { Gage } \\
\text { height }\end{array}$ & Discharge & Hour & $\begin{array}{c}\text { Gage } \\
\text { height }\end{array}$ & Discharge & Hour & $\begin{array}{c}\text { Gage } \\
\text { height }\end{array}$ & Discharge & Hour & $\begin{array}{c}\text { Gage } \\
\text { height }\end{array}$ & Discharge \\
\hline $\begin{array}{r}6 \mathrm{a} \\
7 \\
8 \\
9 \\
10 \\
11 \\
\mathrm{~N} \\
1 \\
2 \\
4 \\
6 \\
8 \\
10 \\
12 \\
\\
4 \\
8 \\
\mathbf{N} \\
4 \\
8 \\
12 \\
7 \\
9 \\
10 \\
11 \\
12 \\
\\
1 \\
2 \\
3 \\
4 \\
5 \\
6 \\
7 \\
8 \\
9 \\
10 \\
11 \\
\mathrm{~N} \\
\end{array}$ & $\begin{array}{l}2.10 \\
2.10 \\
3.87 \\
4.70 \\
4.64 \\
4.60 \\
4.75 \\
5.00 \\
5.13 \\
5.15 \\
5.00 \\
4.44 \\
3.77 \\
3.38 \\
3.14 \\
2.67 \\
2.67 \\
2.40 \\
2.34 \\
2.28 \\
2.15 \\
2.07 \\
2.11 \\
20 \\
2.60 \\
3.70 \\
5.00 \\
5.98 \\
6.53 \\
\text { Ju } \\
6.94 \\
7.30 \\
7.65 \\
7.98 \\
8.29 \\
8.59 \\
8.80 \\
8.94 \\
9.00 \\
8.99 \\
8.95 \\
\end{array}$ & 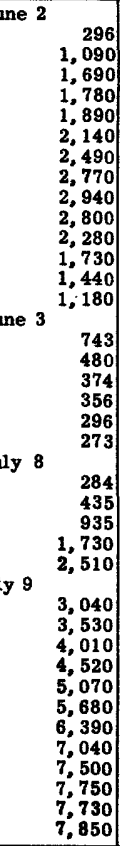 & $\begin{array}{r}1 \\
2 \\
3 \\
4 \\
5 \\
6 \\
7 \\
8 \\
9 \\
10 \\
11 \\
12 \\
2 \\
4 \\
6 \\
8 \\
10 \\
N \\
4 \\
8 \\
12 \\
4 \\
8 \\
\mathrm{~N} \\
4 \\
5 \\
6 \\
7 \\
8 \\
9 \\
10 \\
11 \\
12 \\
12: 30 \\
1 \\
2 \\
4 \\
\end{array}$ & $\begin{array}{l}\text { July } \\
8.90 \\
8.84 \\
8.75 \\
8.62 \\
8.48 \\
8.34 \\
8.16 \\
7.90 \\
7.42 \\
6.78 \\
6.17 \\
5.70 \\
5.07 \\
4.61 \\
4.33 \\
4.16 \\
4.04 \\
3.93 \\
3.55 \\
3.17 \\
2.94 \\
2.77 \\
2.54 \\
2.48 \\
2.59 \\
2.63 \\
2.94 \\
3.54 \\
5.00 \\
7.89 \\
8.40 \\
8.72 \\
9.12 \\
9.24 \\
9.17 \\
9.13 \\
9.04\end{array}$ & $\begin{array}{r}9 \text { (con.) } \\
7,520 \\
7,370 \\
7,180 \\
6,890 \\
6,610 \\
6,330 \\
6,020 \\
5,590 \\
4,910 \\
4,140 \\
3,510 \\
3,060 \\
\text { July } 10 \\
2,520 \\
2,170 \\
1,980 \\
1,870 \\
1,790 \\
1,720 \\
1,490 \\
1,260 \\
1,120 \\
\text { July } 11 \\
1,030 \\
9,905 \\
870 \\
920 \\
935 \\
1,100 \\
1,440 \\
2,440 \\
5,570 \\
6,470 \\
7,160 \\
8,210 \\
\text { July } 12 \\
8,550 \\
8,330 \\
8,210 \\
7,950\end{array}$ & $\begin{array}{r}6 \\
8 \\
10 \\
11 \\
12 \\
1 \\
2 \\
3 \\
4 \\
5 \\
6 \\
7 \\
8 \\
10 \\
N \\
1 \\
4 \\
5 \\
6 \\
7 \\
8 \\
9 \\
10 \\
11 \\
12 \\
\\
1 \\
2 \\
3 \\
4 \\
5 \\
6 \\
9 \\
N \\
4 \\
8 \\
12\end{array}$ & $\begin{array}{l}\text { July } \\
8.95 \\
8.92 \\
8.80 \\
8.68 \\
8.47 \\
7.92 \\
7.14 \\
6.32 \\
5.71 \\
5.26 \\
4.90 \\
4.64 \\
4.44 \\
4.14 \\
3.88 \\
\text { Ju } \\
2.30 \\
2.66 \\
2.91 \\
4.66 \\
5.61 \\
5.99 \\
6.46 \\
7.00 \\
7.36 \\
7.56 \\
7.55 \\
7.55 \\
7.61 \\
7.61 \\
7.44 \\
7.09 \\
5.69 \\
4.57 \\
3.91 \\
3.56 \\
3.30\end{array}$ & 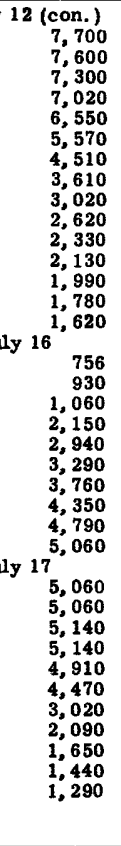 & $\begin{array}{r}4 \\
6 \\
8 \\
10 \\
N \\
1 \\
2 \\
3 \\
4 \\
5 \\
6 \\
8 \\
10 \\
12 \\
\\
1: 3 C \\
6 \\
9 \\
11 \\
N \\
1 \\
2 \\
3 \\
4 \\
5 \\
6 \\
8 \\
10 \\
12 \\
4 \\
8 \\
10 \\
N \\
6 \\
12 \\
6 \\
N\end{array}$ & $\begin{array}{r}\text { Jर } \\
2.89 \\
2.95 \\
3,39 \\
3.77 \\
5.78 \\
6.57 \\
7.04 \\
7.41 \\
7.65 \\
7.96 \\
8.21 \\
8.85 \\
9.40 \\
9.58 \\
\mathrm{~J} \\
9.64 \\
9.51 \\
9.57 \\
9.41 \\
9.17 \\
8.61 \\
7.47 \\
6.58 \\
6.04 \\
5.65 \\
5.38 \\
4.89 \\
4.56 \\
4.30 \\
\mathrm{~J} \\
3.89 \\
3.50 \\
3.55 \\
3.55 \\
3.41 \\
3.20 \\
2.98 \\
2.79\end{array}$ & 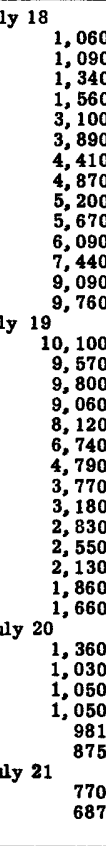 \\
\hline
\end{tabular}


Loup River power canal near Genoa, Nebr.

Location. --Lat $41^{\circ} 25^{\prime}$, long. 97:46', in sec. 28, T, 17 N., R, 4 W., at skimming weir 2 miles downstream from point of diversion and $3 \frac{1}{2}$ miles southwest of Genoa.

Gage-height record. -- Water-stage recorder graph except May 20-31, June 17-20, July 24-27, when gage heights were computed from graphs based on staff-gage readings obtained every 8 hours, or more frequently.

Discharge record. --Stage-discharge relation defined by current-meter measurements.

Maxima,--May-July 1950: Daily discharge, 3,000 cfs May 21.

1936 to April 1950: Daily discharge, 3,410 cfs Apr. 27, 1944.

Remarks. --Canal diverts from Loup River in sec. 6, T. 16 N., R. 4.W. Water is used in power planta near Monroe and Columbus and is returned to Platte River $1 \frac{1}{2}$ miles downstream from Loup River. Diversion began Dec. 2, 1936.

Mean discharge, in cubic feet per second, 1950

\begin{tabular}{|c|c|c|c|c|c|c|c|c|c|c|c|}
\hline Day & May & June & July & Day & May & June & July & Day & May & June & July \\
\hline $\begin{array}{r}1 \\
2 \\
3 \\
4 \\
5 \\
6 \\
7 \\
8 \\
9 \\
10\end{array}$ & $\begin{array}{l}2,600 \\
2,430 \\
2,260 \\
2,290 \\
2,440 \\
2,720 \\
2,630 \\
2,810 \\
2,320 \\
2,790\end{array}$ & $\begin{array}{l}2,240 \\
2,500 \\
2,280 \\
2,160 \\
2,020 \\
1,910 \\
1,930 \\
1,860 \\
1,780 \\
1,780\end{array}$ & $\begin{array}{l}1,320 \\
1,340 \\
1,930 \\
1,980 \\
1,680 \\
1,710 \\
1,870 \\
1,790 \\
1,390 \\
1,950\end{array}$ & $\begin{array}{l}11 \\
12 \\
13 \\
14 \\
15 \\
16 \\
17 \\
18 \\
19 \\
20\end{array}$ & $\begin{array}{l}2,650 \\
2,490 \\
2,600 \\
2,700 \\
2,510 \\
2,400 \\
2,310 \\
2,320 \\
2,720 \\
2,880\end{array}$ & $\begin{array}{l}1,710 \\
1,610 \\
1,470 \\
1,590 \\
2,200 \\
2,010 \\
2,200 \\
2,490 \\
2,520 \\
2,400\end{array}$ & $\begin{array}{l}2,650 \\
1,050 \\
2,240 \\
2,610 \\
2,540 \\
2,490 \\
1,770 \\
1,930 \\
1,370 \\
2,560\end{array}$ & $\begin{array}{l}21 \\
22 \\
23 \\
24 \\
25 \\
26 \\
27 \\
28 \\
29 \\
30 \\
31\end{array}$ & $\begin{array}{l}3,000 \\
2,880 \\
2,600 \\
2,390 \\
2,490 \\
2,650 \\
2,550 \\
2,680 \\
2,810 \\
2,880 \\
2,550\end{array}$ & $\begin{array}{c}2,140 \\
1,900 \\
1,700 \\
1,650 \\
1,630 \\
1,450 \\
1,390 \\
1,380 \\
1,410 \\
1,350 \\
-\end{array}$ & $\begin{array}{l}2,750 \\
2,760 \\
2,180 \\
1,800 \\
2,040 \\
2,010 \\
1,970 \\
2,030 \\
2,070 \\
2,020 \\
1,980\end{array}$ \\
\hline \multicolumn{9}{|c|}{$\begin{array}{l}\text { Monthly mean discharge, in cfs } \ldots \ldots \ldots \ldots \ldots \ldots \ldots \ldots \ldots \ldots \ldots \ldots \ldots \ldots \ldots \ldots \ldots \\
\text { Runoff, in acre-feet. } \ldots \ldots \ldots \ldots \ldots \ldots \ldots \ldots \ldots \ldots \ldots \ldots \ldots \ldots \ldots\end{array}$} & $\begin{array}{r}2,592 \\
159,400\end{array}$ & $\begin{array}{r}1,889 \\
112,400\end{array}$ & $\begin{array}{r}1,993 \\
122,500 \\
\end{array}$ \\
\hline
\end{tabular}


Beaver Creek at Loretto, Nebr.

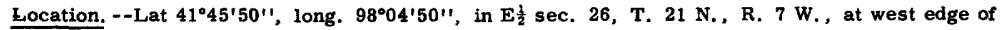
Loretto just downstream from county highway bridge.

Drainage area. $--311 \mathrm{sq} \mathrm{mi}$.

Gage-height record, --Water-stage recorder graph.

Discharge record. --Stage-discharge relation defined by current-meter measurements. Shifting-control method used.

Maxima. --May-July 1956: Discharge, 4,570 cfs 11 a. m. June 2 (gage height, $11.74 \mathrm{ft}$ ).

1944 to April 1950: Discharge recorded, $807 \mathrm{cfs}$ Feb. 28, 1948 (gage height, $9.81 \mathrm{ft}$ ), may have been exceeded by flood in May 1945 for which no record was obtained.

Mean discharge, in cubic feet per second, 1950

\begin{tabular}{|c|c|c|c|c|c|c|c|c|c|c|c|}
\hline Day & May & June & Julý & Day & May & June & July & Day & May & June & July \\
\hline $\begin{array}{r}1 \\
2 \\
3 \\
4 \\
5 \\
6 \\
7 \\
8 \\
9 \\
10\end{array}$ & \begin{tabular}{r|}
82 \\
77 \\
75 \\
72 \\
76 \\
101 \\
103 \\
104 \\
154 \\
167
\end{tabular} & $\begin{array}{r}66 \\
1,810 \\
929 \\
677 \\
481 \\
288 \\
165 \\
119 \\
95 \\
80\end{array}$ & $\begin{array}{r}47 \\
46 \\
47 \\
47 \\
53 \\
50 \\
46 \\
146 \\
1,620 \\
353\end{array}$ & $\begin{array}{l}11 \\
12 \\
13 \\
14 \\
15 \\
16 \\
17 \\
18 \\
19 \\
20\end{array}$ & $\begin{array}{r}136 \\
111 \\
133 \\
124 \\
102 \\
91 \\
88 \\
85 \\
110 \\
126\end{array}$ & $\begin{array}{r}72 \\
66 \\
60 \\
64 \\
86 \\
73 \\
64 \\
82 \\
149 \\
137\end{array}$ & $\begin{array}{r}119 \\
616 \\
165 \\
103 \\
94 \\
584 \\
1,200 \\
607 \\
805 \\
507\end{array}$ & $\begin{array}{l}21 \\
22 \\
23 \\
24 \\
25 \\
26 \\
27 \\
28 \\
29 \\
30 \\
31\end{array}$ & $\begin{array}{r}141 \\
124 \\
102 \\
99 \\
81 \\
777 \\
77 \\
75 \\
78 \\
86 \\
74\end{array}$ & $\begin{array}{l}91 \\
76 \\
71 \\
66 \\
60 \\
55 \\
52 \\
52 \\
50 \\
48 \\
-\end{array}$ & $\begin{array}{r}404 \\
197 \\
426 \\
348 \\
424 \\
328 \\
190 \\
134 \\
105 \\
91 \\
82\end{array}$ \\
\hline \multicolumn{9}{|c|}{$\begin{array}{l}\text { Monthly mean discharge, in cfs. } \ldots \ldots \ldots \ldots \ldots \ldots \ldots \ldots \ldots \ldots \ldots \ldots \ldots \ldots \ldots \ldots \ldots \\
\text { Runoff, in inches. } \ldots \ldots \ldots \ldots \ldots \ldots \ldots \ldots \ldots \ldots \ldots \ldots \ldots\end{array}$} & 8,210 & 12,206 & $\begin{array}{r}322 \\
19,800\end{array}$ \\
\hline
\end{tabular}

Gage height, in feet, and discharge, in cubic feet per second, at indicated time, 1950

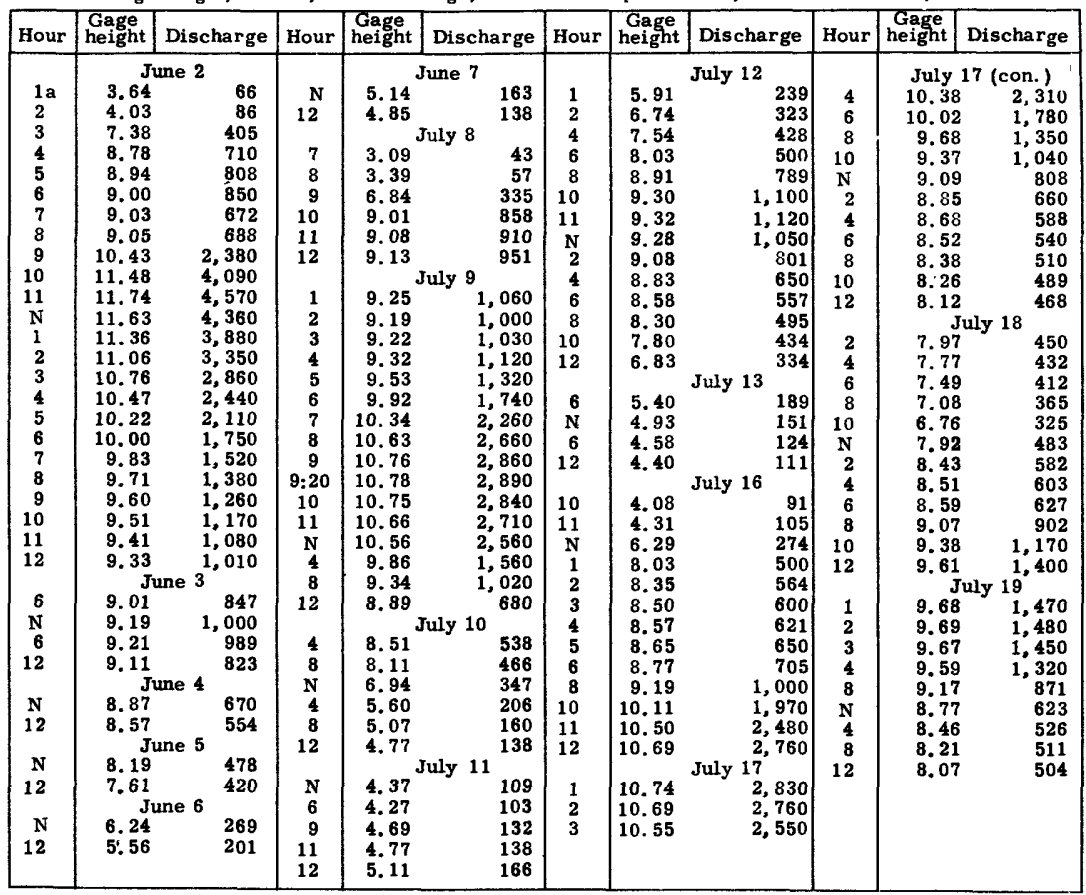


Beaver Creek at Genoa, Nebr.

Location. --Lat $41^{\circ} 26^{\prime} 20^{\prime \prime}$, long. $97^{\circ} 44^{\prime} 30^{\prime \prime}$, in $\mathrm{W}_{2} \mathrm{SE} \frac{1}{2}$ sec. 14, T. 17 N., R. 4 W., at bridge on State Highway 22, a fifth of a mile upstream from Union Pacific Railroad bridge, 1 mile southwest of Genoa and about 3 miles upstream from mouth.

Drainage area. $--627 \mathrm{sq} \mathrm{mi}$, of which about $410 \mathrm{sq} \mathrm{mi}$ contribute directly to surface runoff.

Gage-height record. --Water-stage recorder graph except June 23-27 when twice-daily chain-gage readings were used. Shifting-control method used May 1 to 6 p. m. June 2; June 7 to 3 a. m., July 9.

Discharge record. - -Stage-discharge relation defined by current-meter measurements below 8,500 cfs and extended to peak stage.

Maxima. --May-July 1950: Discharge, 21, 200 cfs 2:30 a. m. July 19 (gage height, $18.70 \mathrm{ft}$ ).

1940 to April 1950: Discharge, $3,930 \mathrm{cfs} J u n e$ 21, 1942 (gage height, $15.5 \mathrm{ft}$, from floodmarks ).

Remarks. - - Natural flow affected slightly by ground-water withdrawals for irrigation. Diurnal fluctuation at low flow caused by power plants above station.

Mean discharge, in cubic feet per second, 1950

\begin{tabular}{|c|c|c|c|c|c|c|c|c|c|c|c|}
\hline Day & May & June & July & Day & May & June & July & Day & May & June & July \\
\hline $\begin{array}{r}1 \\
2 \\
3 \\
4 \\
5 \\
6 \\
7 \\
8 \\
9 \\
10\end{array}$ & $\begin{array}{l}107 \\
122 \\
118 \\
111 \\
110 \\
104 \\
104 \\
145 \\
493 \\
379\end{array}$ & $\begin{array}{r}122 \\
2,220 \\
4,100 \\
2,070 \\
1,150 \\
842 \\
591 \\
322 \\
222 \\
171\end{array}$ & $\begin{array}{r}83 \\
82 \\
84 \\
85 \\
90 \\
80 \\
84 \\
86 \\
4,160 \\
3,020\end{array}$ & $\begin{array}{l}11 \\
12 \\
13 \\
14 \\
15 \\
16 \\
17 \\
18 \\
19 \\
20\end{array}$ & $\begin{array}{l}207 \\
194 \\
160 \\
145 \\
164 \\
152 \\
125 \\
115 \\
118 \\
136\end{array}$ & $\begin{array}{l}141 \\
124 \\
118 \\
117 \\
118 \\
122 \\
149 \\
150 \\
155 \\
138\end{array}$ & $\begin{array}{r}1,780 \\
2,570 \\
1,690 \\
773 \\
321 \\
243 \\
1,340 \\
3,410 \\
10,000 \\
2,160\end{array}$ & $\begin{array}{l}21 \\
22 \\
23 \\
24 \\
25 \\
26 \\
27 \\
28 \\
29 \\
30 \\
31\end{array}$ & $\begin{array}{l}144 \\
157 \\
168 \\
152 \\
165 \\
140 \\
112 \\
111 \\
121 \\
122 \\
124\end{array}$ & $\begin{array}{r}174 \\
157 \\
122 \\
109 \\
102 \\
91 \\
89 \\
88 \\
86 \\
84 \\
- \\
\end{array}$ & $\begin{array}{r}965 \\
929 \\
1,260 \\
866 \\
521 \\
465 \\
495 \\
366 \\
263 \\
220 \\
191 \\
\end{array}$ \\
\hline Ior & & & & & & & & & $\begin{array}{r}156 \\
9,570\end{array}$ & $\begin{array}{r}475 \\
28,250\end{array}$ & $\begin{array}{r}1,248 \\
76,720\end{array}$ \\
\hline
\end{tabular}

Gage height, in feet, and discharge, in cubic feet per second, at indicated time, 1950

\begin{tabular}{|c|c|c|c|c|c|c|c|c|c|c|c|}
\hline Hour & $\begin{array}{l}\text { Gage } \\
\text { height }\end{array}$ & Discharge & lour & $\begin{array}{l}\text { Gage } \\
\text { eight }\end{array}$ & Discharge & Iour & $\begin{array}{l}\text { Gage } \\
\text { height }\end{array}$ & Discharge & Hour & & Discharge \\
\hline $\begin{array}{r}4 \\
5 \\
6 \\
7 \\
8 \\
9 \\
10 \\
11 \\
N \\
1 \\
2 \\
3 \\
4 \\
5 \\
6 \\
7 \\
8 \\
9 \\
10 \\
11 \\
12 \\
\\
4 \\
8 \\
N \\
4 \\
8 \\
11 \\
2 \\
2 \\
5 \\
8 \\
5 \\
12 \\
6 \\
6 \\
6\end{array}$ & $\begin{array}{l}1.44 \\
1.56 \\
1.60 \\
1.73 \\
2.63 \\
2.92 \\
3.90 \\
4.85 \\
5.70 \\
5.97 \\
5.87 \\
5.96 \\
6.83 \\
8.70 \\
11.50 \\
14.10 \\
15.45 \\
16.02 \\
16.11 \\
16.00 \\
15.85 \\
15 \\
5.15 \\
4.05 \\
12.70 \\
1.17 \\
9.86 \\
9.36 \\
9.62 \\
9.86 \\
9.63 \\
8.97 \\
7.80 \\
6.75 \\
5.96 \\
5.28 \\
4.81 \\
4.32 \\
J\end{array}$ & 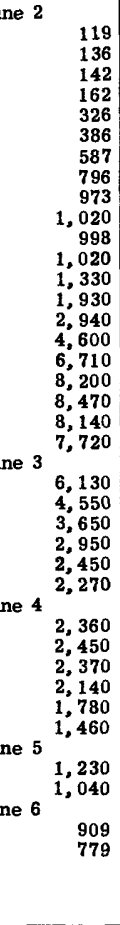 & $\begin{array}{r}6 \\
6 \\
6 \\
6 \\
9 \\
10 \\
11 \\
12 \\
\\
1 \\
2 \\
3 \\
4 \\
5 \\
6 \\
7 \\
8 \\
9: 30 \\
11 \\
1 \\
3 \\
5 \\
7 \\
10 \\
12 \\
1 \\
2 \\
4 \\
6 \\
9 \\
N \\
4 \\
8 \\
12 \\
4 \\
8 \\
N \\
4 \\
8 \\
12\end{array}$ & \begin{tabular}{|l}
3.90 \\
3.38 \\
2.67 \\
2.33 \\
1.24 \\
1.33 \\
1.41 \\
1.47 \\
1.51 \\
3.60 \\
8.30 \\
11.55 \\
12.87 \\
13.08 \\
13.51 \\
15.51 \\
16.32 \\
15.99 \\
15.15 \\
14.14 \\
13.08 \\
11.84 \\
10.00 \\
9.16 \\
9.32 \\
9.83 \\
11.74 \\
12.79 \\
12.99 \\
12.38 \\
11.10 \\
9.82 \\
9.04 \\
8.53 \\
8.38 \\
8.11 \\
7.53 \\
6.70 \\
6.06
\end{tabular} & 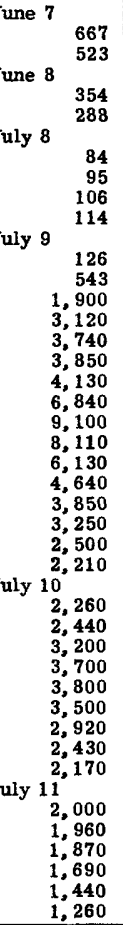 & $\begin{array}{r}2 \\
3 \\
4 \\
5 \\
6 \\
7 \\
8 \\
9 \\
10 \\
11 \\
\mathrm{~N} \\
1 \\
2 \\
3 \\
4 \\
6 \\
9 \\
12 \\
2 \\
5 \\
8 \\
\mathrm{~N} \\
6 \\
12 \\
\\
6 \\
\mathrm{~N} \\
6 \\
12 \\
6 \\
6 \\
6 \\
11 \\
12 \\
2 \\
4 \\
8 \\
10\end{array}$ & $\begin{array}{l}5.97 \\
6.01 \\
6.57 \\
7.98 \\
9.13 \\
9.66 \\
9.70 \\
9.64 \\
10.00 \\
11.37 \\
12.62 \\
13.11 \\
13.20 \\
13.17 \\
12.88 \\
11.79 \\
9.79 \\
8.69 \\
8.56 \\
\mathbf{J} \\
8.61 \\
8.43 \\
7.82 \\
6.50 \\
5.44 \\
4.77 \\
4.41 \\
3.76 \\
2.93 \\
2.46 \\
2.06 \\
1.88 \\
1.82 \\
1.97 \\
3.44 \\
4.69 \\
5.45 \\
5.96 \\
6.02\end{array}$ & 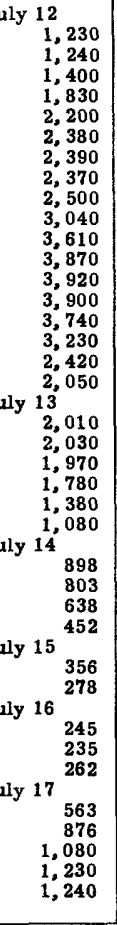 & 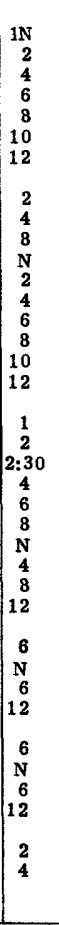 & \begin{tabular}{|c} 
July \\
5.87 \\
5.76 \\
6.06 \\
7.13 \\
8.54 \\
9.82 \\
10.64 \\
\multicolumn{1}{c}{$\mathrm{J}$} \\
10.92 \\
10.71 \\
9.79 \\
8.99 \\
9.53 \\
10.40 \\
11.35 \\
13.30 \\
15.55 \\
16.90 \\
\\
17.80 \\
18.55 \\
18.70 \\
18.34 \\
17.73 \\
17.18 \\
16.21 \\
15.18 \\
14.12 \\
12.92 \\
10.35 \\
8.38 \\
7.16 \\
6.47 \\
5.67 \\
4.86 \\
4.27 \\
3.98 \\
3.95 \\
4.07
\end{tabular} & $\begin{array}{r}17 \text { (con.) } \\
1,200 \\
1,170 \\
1,260 \\
1,570 \\
2,010 \\
2,430 \\
2,730 \\
\text { uly } 18 \\
2,850 \\
2,760 \\
2,420 \\
2,150 \\
2,330 \\
2,640 \\
3,030 \\
3,980 \\
6,940 \\
11,100 \\
19 \\
15,400 \\
20,200 \\
21,200 \\
18,700 \\
15,000 \\
12,300 \\
8,770 \\
6,180 \\
4,620 \\
3,760 \\
2,620 \\
1,960 \\
1,580 \\
1,370 \\
121,150 \\
1,70 \\
20\end{array}$ \\
\hline
\end{tabular}


Shell Creek at New man Grove, Nebr.

Location. --iat $41^{\circ} 44^{\prime} 30^{\prime \prime}$, long. $97^{\circ} 45^{\prime} 00^{\prime \prime}$, in $\mathrm{NW} \frac{1}{4} \mathrm{NW} \frac{1}{4}$ sec. 2, T. $20 \mathrm{~N}$., R. 4 W., at bridge on county road, 1 twile east of Newman Grove and 5 miles downstream from North Branch.

Drainage area. $--122 \mathrm{sq} \mathrm{mi}$.

Gage-height record. --Water-stage recorder graph except period July 19-31 when graph based on twice daily wire-weight gage readings was used.

Discharge record. --Stage-discharge relation defined by current-meter measurements below 800 cfs and extended to peak stage on baais of computation of combined flow through bridge and over road embankments during nood of June 2, 1950.

Maximum. --May-July 1950: Discharge, 12, 000 cfs 1 p. m. July 18 (gage height, $20.20 \mathrm{ft}$ ).

Remarks. --Station established July 1949; no previous flood data available.

Mean discharge, in cubic feet per second, 1950

\begin{tabular}{|c|c|c|c|c|c|c|c|c|c|c|c|}
\hline Day & April & May & June & Day & April & May & June & Day & April & May & June \\
\hline $\begin{array}{r}1 \\
2 \\
3 \\
4 \\
5 \\
6 \\
7 \\
8 \\
9 \\
10\end{array}$ & $\begin{array}{r}2.5 \\
2.4 \\
2.4 \\
2.4 \\
2.6 \\
2.8 \\
2.7 \\
4.2 \\
42 \\
2.2\end{array}$ & $\begin{array}{r}1.0 \\
5,050 \\
76 \\
11 \\
8.0 \\
6.9 \\
6.5 \\
6.0 \\
4.8 \\
2.5\end{array}$ & $\begin{array}{c}1.1 \\
1.2 \\
1.2 \\
1.2 \\
1.3 \\
1.3 \\
1.2 \\
1.5 \\
590 \\
12\end{array}$ & $\begin{array}{l}11 \\
12 \\
13 \\
14 \\
15 \\
16 \\
17 \\
18 \\
19 \\
20\end{array}$ & \begin{tabular}{l|}
1.2 \\
1.1 \\
1.1 \\
1.1 \\
1.1 \\
1.0 \\
1.0 \\
1.1 \\
1.1 \\
2.0
\end{tabular} & $\begin{array}{c}2.3 \\
2.6 \\
2.7 \\
29 \\
6.9 \\
3.1 \\
2.6 \\
178 \\
4.8 \\
1.9\end{array}$ & $\begin{array}{r}113 \\
311 \\
15 \\
6.6 \\
5.7 \\
942 \\
304 \\
4,580 \\
1,570 \\
86\end{array}$ & $\begin{array}{l}21 \\
22 \\
23 \\
24 \\
25 \\
26 \\
27 \\
28 \\
29 \\
30 \\
31 \\
\end{array}$ & $\begin{array}{l}1.2 \\
1.0 \\
1.0 \\
1.9 \\
1.7 \\
1.1 \\
1.0 \\
1.0 \\
1.0 \\
1.1 \\
1.1 \\
\end{array}$ & $\begin{array}{l}1.4 \\
1.3 \\
1.4 \\
1.2 \\
1.2 \\
1.1 \\
1.1 \\
1.1 \\
1.1 \\
1.1 \\
- \\
\end{array}$ & $\begin{array}{c}41 \\
107 \\
324 \\
70 \\
89 \\
61 \\
12 \\
5.8 \\
5.2 \\
4.7 \\
4.4 \\
\end{array}$ \\
\hline \multicolumn{9}{|c|}{ 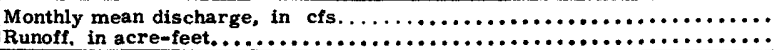 } & $\begin{array}{r}2.99 \\
184\end{array}$ & $\begin{array}{r}181 \\
10,750\end{array}$ & $\begin{array}{r}313 \\
19,240\end{array}$ \\
\hline
\end{tabular}

Gage height, in feet, and discharge, in cubic feet per second, at indicated time, 1950

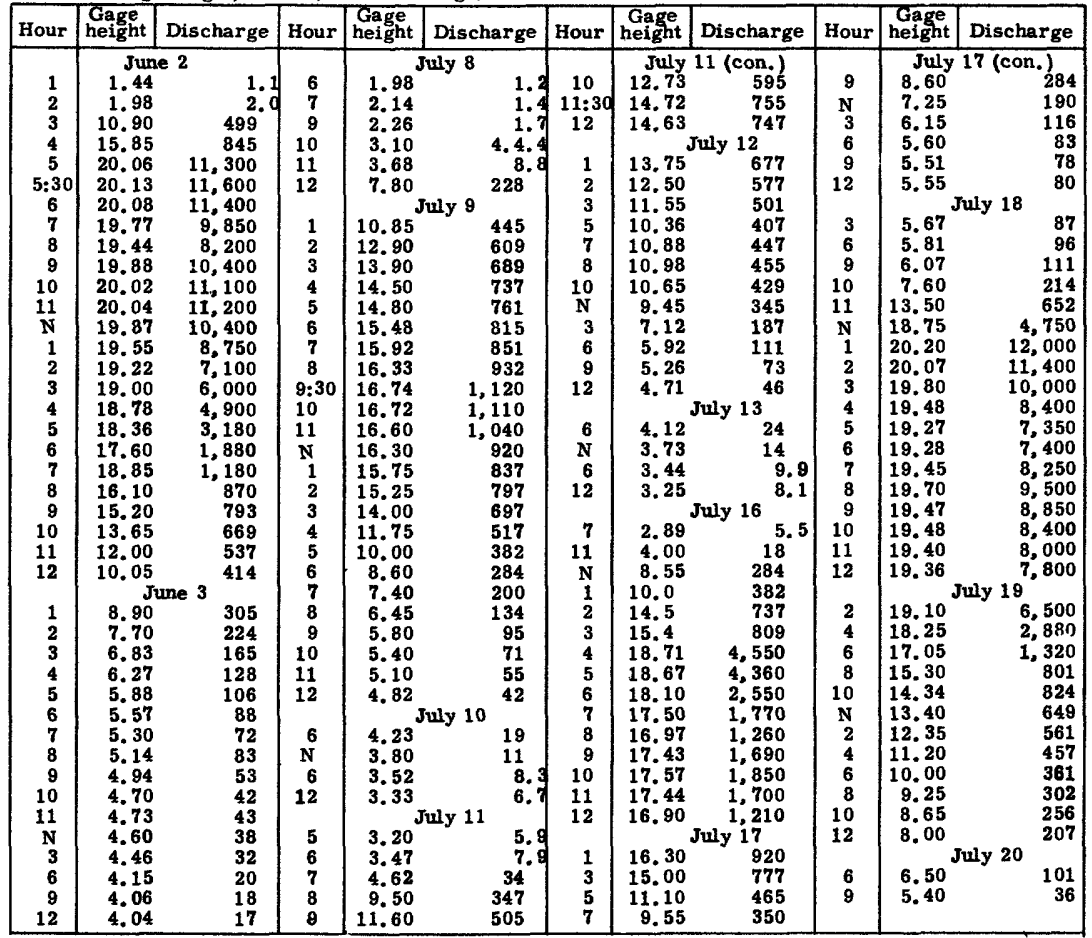


Shell Creek near Columbus, Nebr.

Location, --Lat $40^{\circ} 31^{\prime} 30^{\prime \prime}$, long. $97^{\circ} 17^{\prime} 00^{\prime \prime}$ in sec. 23, T. 18 N., R. 1 E., 80 ft upstream from county highway bridge, 1 mile upstream from Loseke Creek and 7 miles northeast of Columbus.

Drainage area. $-\mathbf{- 2 7 0} \mathrm{sq} \mathbf{m i}$.

Gage-height record.--Water-stage recorder graph except July 8, 9, 19-21. Gage heights for period .July 19-21 computed from graph based on fragmentary periods of water-stage recorder graph and wire-weight gage readings.

Discharge record. --Stage-discharge relation defined by current-meter measurements. Shiftingcontrol method used. Discharge for July 8, 9, interpolated.

Maxima. --May-July 1950: Discharge, 5,970 cfs 12:30 p. m. June 3 (gage height, $21.38 \mathrm{ft}$ ).

1947 to April 1950: Discharge, 4, 600 cfs June 2, 1947 (gage height, $21.7 \mathrm{ft}$ ).

Mean discharge, in cubic feet per second, 1950

\begin{tabular}{|c|c|c|c|c|c|c|c|c|c|c|c|}
\hline Day & May & June & July & Day & May & June & July & Day & May & June & July \\
\hline $\begin{array}{r}1 \\
2 \\
3 \\
4 \\
5 \\
6 \\
7 \\
8 \\
9 \\
10\end{array}$ & $\begin{array}{r}30 \\
26 \\
23 \\
22 \\
24 \\
25 \\
28 \\
30 \\
84 \\
390\end{array}$ & $\begin{array}{r}22 \\
410 \\
4,510 \\
2,780 \\
671 \\
121 \\
68 \\
52 \\
44 \\
40\end{array}$ & $\begin{array}{l}14 \\
13 \\
12 \\
12 \\
12 \\
11 \\
11 \\
11 \\
11 \\
33\end{array}$ & $\begin{array}{l}11 \\
12 \\
13 \\
14 \\
15 \\
16 \\
17 \\
18 \\
19 \\
20\end{array}$ & $\begin{array}{r}113 \\
48 \\
32 \\
26 \\
24 \\
21 \\
20 \\
19 \\
22 \\
20\end{array}$ & $\begin{array}{r}36 \\
34 \\
83 \\
35 \\
30 \\
28 \\
49 \\
46 \\
82 \\
214\end{array}$ & $\begin{array}{r}458 \\
167 \\
334 \\
389 \\
62 \\
35 \\
33 \\
486 \\
1,160 \\
3,140\end{array}$ & $\begin{array}{l}21 \\
22 \\
23 \\
24 \\
25 \\
26 \\
27 \\
28 \\
29 \\
30 \\
31\end{array}$ & $\begin{array}{l}22 \\
28 \\
26 \\
20 \\
18 \\
18 \\
17 \\
22 \\
22 \\
47 \\
32\end{array}$ & $\begin{array}{l}75 \\
41 \\
30 \\
26 \\
23 \\
20 \\
18 \\
18 \\
16 \\
15 \\
-\end{array}$ & $\begin{array}{r}2,360 \\
411 \\
611 \\
525 \\
429 \\
108 \\
125 \\
113 \\
102 \\
62 \\
50\end{array}$ \\
\hline \multicolumn{9}{|c|}{ 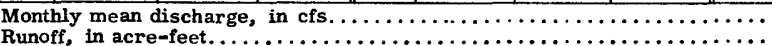 } & $\begin{array}{r}41.9 \\
2,580\end{array}$ & $\begin{array}{r}320 \\
19,020\end{array}$ & $\begin{array}{r}365 \\
22,410\end{array}$ \\
\hline
\end{tabular}

Gage height, in feet, and discharge, in cubic feet per second, at indicated time, 1950

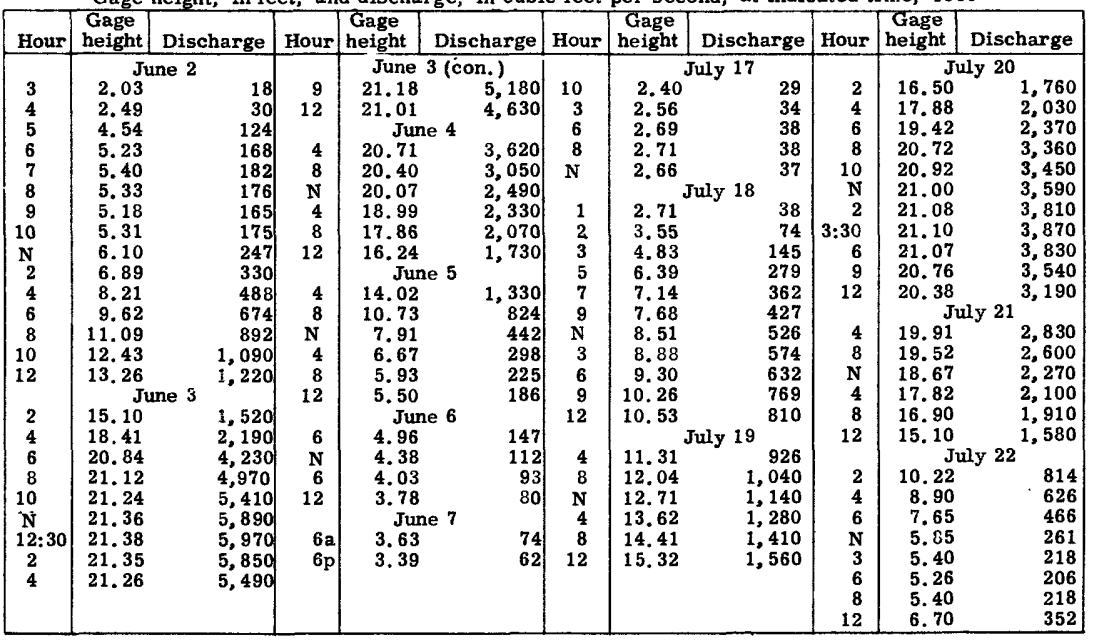


Elkhorn River at Norfolk, Nebr.

Location. --Lat $42^{\circ} 00^{\prime} 10^{\prime \prime}$, long. $97^{\circ} 25^{\prime} 30^{\prime \prime}$, in NW $\frac{1}{4} \mathrm{NW} \frac{1}{4}$ sec. 3, T. $23 \mathrm{~N}$., R. 1 W., at bridge on U. S. Highway 81,1 mile south of Norfolk and $33 / 4$ miles upstream from North Branch.

Drainage area., $--2,790 \mathrm{sq} \mathrm{mi}$, of which only about $2,400 \mathrm{sq} \mathrm{mi}$ contribute directly to surface runoff.

Gage-height record. --Wire-weight gage read twice daily. Mean daily gage heights July 19, 20, computed from graph based on gage readings.

Discharge record. --Stage-di scharge relation defined by current-meter measurements. Shifting-control method used.

Maxima, --May-June 1950: Discharge ohserved, 7, 080 cfs 9 a. m. July 19 (gage height, 8. $35 \mathrm{ft}$ ). 1945 to April 1950: Discharge, 12,600 cfs June 26, 1947; gage height, 12. $25 \mathrm{ft}$ Feb. 28, 1948 (backwater from ice).

Mean discharge, in cubic feet per second, 1950

\begin{tabular}{|c|c|c|c|c|c|c|c|c|c|c|c|}
\hline Day & May & June & July & Day & May & June & July & Day & May & June & July \\
\hline $\begin{array}{r}1 \\
2 \\
3 \\
4 \\
5 \\
6 \\
7 \\
8 \\
9 \\
10\end{array}$ & $\begin{array}{r}582 \\
592 \\
639 \\
671 \\
694 \\
740 \\
787 \\
976 \\
1,290 \\
1,330\end{array}$ & $\begin{array}{r}1,020 \\
1,300 \\
2,380 \\
2,250 \\
1,660 \\
1,420 \\
1,260 \\
1,090 \\
983 \\
902\end{array}$ & $\begin{array}{l}441 \\
415 \\
381 \\
381 \\
415 \\
406 \\
415 \\
394 \\
607 \\
902\end{array}$ & $\begin{array}{l}11 \\
12 \\
13 \\
14 \\
15 \\
16 \\
17 \\
18 \\
19 \\
20\end{array}$ & $\begin{array}{r}1,460 \\
1,410 \\
1,340 \\
1,270 \\
1,070 \\
990 \\
969 \\
997 \\
1,130 \\
1,170\end{array}$ & $\begin{array}{r}909 \\
856 \\
775 \\
740 \\
1,050 \\
936 \\
769 \\
1,000 \\
1,160 \\
1,130\end{array}$ & $\begin{array}{l}1,110 \\
1,730 \\
1,570 \\
1,440 \\
1,560 \\
1,690 \\
1,840 \\
1,790 \\
5,300 \\
2,810\end{array}$ & $\begin{array}{l}21 \\
22 \\
23 \\
24 \\
25 \\
26 \\
27 \\
28 \\
29 \\
30 \\
31\end{array}$ & $\begin{array}{r}1,690 \\
1,510 \\
1,320 \\
1,140 \\
1,080 \\
1983 \\
915 \\
863 \\
850 \\
876 \\
1,000 \\
\end{array}$ & $\begin{array}{r}1,200 \\
1,260 \\
1,360 \\
1,300 \\
1,120 \\
902 \\
751 \\
607 \\
547 \\
491 \\
- \\
\end{array}$ & $\begin{array}{l}2,980 \\
2,39 \\
2,890 \\
2,360 \\
1,900 \\
1,900 \\
1,460 \\
1,310 \\
1,140 \\
1,020 \\
889\end{array}$ \\
\hline \multicolumn{9}{|c|}{$\begin{array}{l}\text { Monthly mean discharge, in cfs. } \ldots \ldots \ldots \ldots \ldots \ldots \ldots \ldots \ldots \ldots \ldots \ldots \ldots \ldots \ldots \ldots \\
\text { Runoff, in acre-feet. } \ldots \ldots \ldots \ldots \ldots \ldots \ldots \ldots \ldots \ldots \ldots \ldots \ldots\end{array}$} & $\begin{array}{r}1,043 \\
64,130\end{array}$ & $\begin{array}{r}1,104 \\
65,710\end{array}$ & $\begin{array}{r}1,47^{\circ} \\
90,910\end{array}$ \\
\hline
\end{tabular}


Elkhorn River at Waterloo, Nebr.

I.ocation. --Lat $41^{\circ} 19^{\prime} 50^{\prime \prime}$, long. $96^{\circ} 17^{\prime} 50^{\prime \prime}$, in SE $\frac{1}{4}$ sec. 21, T. 16 N., R. 10 E., just downstream from Rawhide Creek and $3 \frac{1}{2}$ miles north of Waterloo.

I "ainage area. $--6,900 \mathrm{sq} \mathrm{mi}$, of which about $6,500 \mathrm{sq} \mathrm{mi}$ contribute directly to surface runoff.

Gage-height record, --Water-stage recorder graph except for short periods on June 3, 5, 19, July 15, $16,18-20$, and all day June 20. The periods of missing record were filled in by estimated graphs based on the trend indicated by the water-stage recorder on either side of the periods.

I ischarge record. --Stage-discharge relation defined by current-meter measurements. Shifting-control method used.

1.7xima.--May-July 1950: Discharge, 13,400 cfs 5 a. m. July 20; gage height, $7.08 \mathrm{ft} 11$ a. m. July 22. $1911-13,1928$ to April 1950: Discharge, 100, 000 cfs June 12, 1944 (gage height, 16. $6 \mathrm{ft}$; from floodmark in gage well), from current-metermeasurement of main channel and velocity-area studies of overflow section.

Flood of June 12, 1944 is the greatest known.

Femarks. --Several small diversions above station for irrigation.

Mean discharge, in cubic feet per second, 1950

\begin{tabular}{|c|c|c|c|c|c|c|c|c|c|c|c|}
\hline Day & May & June & July & Day & May & June & July & Day & May & June & July \\
\hline $\begin{array}{r}1 \\
2 \\
3 \\
4 \\
5 \\
6 \\
7 \\
8 \\
9 \\
10\end{array}$ & $\begin{array}{l}1,240 \\
1,180 \\
1,150 \\
1,140 \\
1,460 \\
1,470 \\
1,430 \\
1,790 \\
2,230 \\
2,850\end{array}$ & $\begin{array}{l}1,230 \\
1,300 \\
4,870 \\
6,110 \\
5,820 \\
3,250 \\
2,810 \\
2,530 \\
2,080 \\
1,700\end{array}$ & $\begin{array}{l}935 \\
894 \\
861 \\
829 \\
805 \\
775 \\
758 \\
775 \\
805 \\
811\end{array}$ & $\begin{array}{l}11 \\
12 \\
13 \\
14 \\
15 \\
16 \\
17 \\
18 \\
19 \\
20\end{array}$ & $\begin{array}{l}2,490 \\
2,530 \\
2,410 \\
2,290 \\
2,120 \\
1,840 \\
1,590 \\
1,470 \\
1,430 \\
1,520\end{array}$ & $\begin{array}{l}1,490 \\
1,330 \\
1,180 \\
1,220 \\
1,120 \\
1,050 \\
1,160 \\
1,820 \\
3,670 \\
3,100\end{array}$ & $\begin{array}{r}835 \\
1,430 \\
1,820 \\
3,000 \\
2,930 \\
6,400 \\
3,770 \\
3,710 \\
7,270 \\
11,000\end{array}$ & $\begin{array}{l}21 \\
22 \\
23 \\
24 \\
25 \\
26 \\
27 \\
28 \\
29 \\
30 \\
31 \\
\end{array}$ & $\begin{array}{l}1,780 \\
1,780 \\
2,040 \\
1,930 \\
1,780 \\
1,560 \\
1,500 \\
1,390 \\
1,360 \\
1,310 \\
1,260 \\
\end{array}$ & $\begin{array}{c}2,580 \\
2,790 \\
2,910 \\
2,290 \\
2,380 \\
2,230 \\
1,760 \\
1,350 \\
1,140 \\
1,020 \\
-\end{array}$ & $\begin{array}{r}9,300 \\
10,600 \\
6,250 \\
5,360 \\
4,910 \\
3,500 \\
3,200 \\
2,980 \\
2,370 \\
2,020 \\
2,030 \\
\end{array}$ \\
\hline \multicolumn{9}{|c|}{ 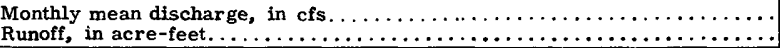 } & $\begin{array}{r}1,720 \\
105,800 \\
\end{array}$ & $\begin{array}{r}2,310 \\
137,400\end{array}$ & $\begin{array}{r}3,320 \\
204,200 \\
\end{array}$ \\
\hline
\end{tabular}

Gage height, in feet, and discharge, in cubic feet per second, at indicated time, 1950

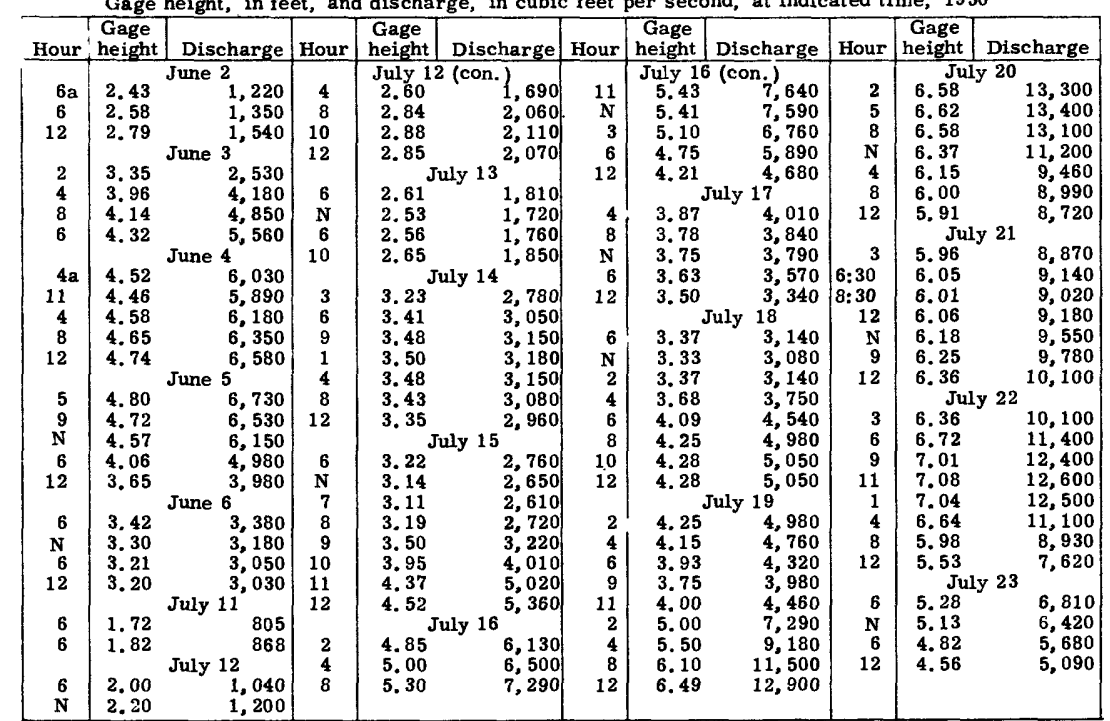


Logan Creek near Uehling, Nebr.

Location. --Lat $41^{\circ} 42^{\prime} 50^{\prime \prime}$, long. $96^{\circ} 31^{\prime} 15^{\prime \prime}$, on south line of $\mathrm{SE} \frac{1}{4} \mathrm{SE} \frac{1}{4} \mathrm{sec} .9, \mathrm{~T}, 20 \mathrm{~N} ., \mathrm{R}, 8 \mathrm{E} .$, at county bridge 2 miles southwest of Uehling and 8 miles upstream from mouth.

Drainage area. $--1,030 \mathrm{sq} \mathrm{mi}$.

Gage-height record. --Water-stage recorder graph except May 10, May 17 to June 1, July 19, when no record was obtained. Record for May 13-16 doubtful because of faulty intake action.

Discharge record. --Stage-discharge relation defined by current-meter measurements. Shifting-control method used. Discharge for periods of no gage-height record computed on basis of discharge measurement made on May 22, fragmentary recorder graph, floodmark on July 19, and weather records.

Maxima. --May-July 1950: Discharge, about 3,860 cfs, July 19 (gage height, $10.3 \mathrm{ft}$, from floodmark). 1941 to April 1950: Discharge, $13,700 \mathrm{cfs}$, June 11, 1944 (gage height, 17, $65 \mathrm{ft}$, from floodmarks), from rating curve extended above $5,800 \mathrm{cfs}$ by velocity-area studies.

Maximum stage known, 18.6 ft.June 5, 1950, from floodmarks (discharge, 20, 000 cfs, from rating curve extended above 5,800 cfs by velocity-area studies).

Mean discharge, in cubic feet per second, 1950

\begin{tabular}{|c|c|c|c|c|c|c|c|c|c|c|c|}
\hline Day & May & June & July & Day & May & June & July & Day & May & June & July \\
\hline $\begin{array}{r}1 \\
2 \\
3 \\
4 \\
5 \\
6 \\
7 \\
8 \\
9 \\
10\end{array}$ & $\begin{array}{r}97 \\
90 \\
89 \\
87 \\
105 \\
109 \\
121 \\
187 \\
328 \\
630\end{array}$ & \begin{tabular}{r|}
100 \\
209 \\
218 \\
124 \\
89 \\
75 \\
72 \\
67 \\
64 \\
61
\end{tabular} & $\begin{array}{l}74 \\
75 \\
76 \\
75 \\
77 \\
77 \\
74 \\
71 \\
79 \\
82\end{array}$ & $\begin{array}{l}11 \\
12 \\
13 \\
14 \\
15 \\
16 \\
17 \\
18 \\
19 \\
20\end{array}$ & $\begin{array}{r}164 \\
118 \\
107 \\
101 \\
95 \\
92 \\
90 \\
130\end{array}$ & $\begin{array}{r}60 \\
64 \\
82 \\
101 \\
77 \\
98 \\
216 \\
1,380 \\
1,150 \\
690\end{array}$ & $\begin{array}{r}74 \\
82 \\
166 \\
97 \\
93 \\
150 \\
113 \\
128 \\
2,300 \\
1,080\end{array}$ & $\begin{array}{l}21 \\
22 \\
23 \\
24 \\
25 \\
26 \\
27 \\
28 \\
29 \\
30 \\
31\end{array}$ & -100 & $\begin{array}{r}342 \\
124 \\
101 \\
123 \\
96 \\
81 \\
79 \\
79 \\
78 \\
77 \\
- \\
\end{array}$ & $\begin{array}{r}98 n \\
1,910 \\
662 \\
305 \\
196 \\
166 \\
152 \\
139 \\
139 \\
116 \\
113 \\
\end{array}$ \\
\hline \multicolumn{9}{|c|}{ 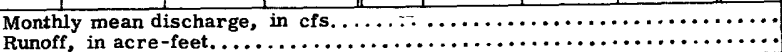 } & 7,970 & $\begin{array}{r}206 \\
12,250\end{array}$ & 19,670 \\
\hline
\end{tabular}

Salt Creek at Lincoln, Nebr.

Location. - Lat $40^{\circ} 50^{\prime} 50^{\prime \prime}$, long. $96^{\circ} 40^{\prime} 50^{\prime \prime}$, in SW $\frac{1}{4}$ sec. 7, T. $10 \mathrm{~N}$, , R. $7 \mathrm{E}$., at bridge on North 27 th Street at north edge of Lincoln, about one mile downstream from Oak Creek.

Drainage area. $--740 \mathrm{sq} \mathrm{mi}$

Gage-height record. --Water-stage recorder graph when stage was above gage height $6.3 \mathrm{ft}$; below this stage, wire-weight gage read twice daily. Gage heights May 5, 8, 11, 25, 29, 31, June 14, 15, July $12,13,31$, were computed from graphs based on gage readings and recorder graph.

Discharge record. --Stage-discharge relation defined by current-meter measurements. Shiftingcontrol method used.

Maxima. --May-July 1950: Discharge, 27,800 cfs 9 a. m. May 9 (gage height, 26. $05 \mathrm{ft}$ ).

Flood of May 9, 1950 greatest since that of July 6, 1908, which may have been equal or greater.

\begin{tabular}{|c|c|c|c|c|c|c|c|c|c|c|c|}
\hline Day & May & June & July & Day & May & June & July & Day & May & June & July \\
\hline $\begin{array}{r}1 \\
2 \\
3 \\
4 \\
5 \\
6 \\
7 \\
8 \\
9 \\
10\end{array}$ & $\begin{array}{r}78 \\
65 \\
68 \\
64 \\
527 \\
226 \\
120 \\
295 \\
16,700 \\
1,860\end{array}$ & $\begin{array}{r}171 \\
127 \\
220 \\
106 \\
102 \\
88 \\
80 \\
77 \\
71 \\
66\end{array}$ & $\begin{array}{l}47 \\
40 \\
50 \\
68 \\
53 \\
50 \\
41 \\
44 \\
82 \\
66\end{array}$ & $\begin{array}{l}11 \\
12 \\
13 \\
14 \\
15 \\
16 \\
17 \\
18 \\
19 \\
20\end{array}$ & $\begin{array}{r}409 \\
285 \\
200 \\
159 \\
124 \\
111 \\
102 \\
96 \\
153 \\
168\end{array}$ & $\begin{array}{r}66 \\
61 \\
62 \\
700 \\
490 \\
100 \\
147 \\
104 \\
129 \\
84\end{array}$ & $\begin{array}{r}69 \\
213 \\
492 \\
109 \\
64 \\
94 \\
106 \\
64 \\
187 \\
86\end{array}$ & $\begin{array}{l}21 \\
22 \\
23 \\
24 \\
25 \\
26 \\
27 \\
28 \\
29 \\
30 \\
31\end{array}$ & $\begin{array}{r}140 \\
117 \\
98 \\
82 \\
688 \\
372 \\
127 \\
102 \\
1,360 \\
1,540 \\
324\end{array}$ & \begin{tabular}{l|}
73 \\
66 \\
62 \\
59 \\
47 \\
51 \\
48 \\
48 \\
45 \\
47 \\
-
\end{tabular} & $\begin{array}{r}62 \\
51 \\
50 \\
51 \\
48 \\
51 \\
48 \\
47 \\
44 \\
40 \\
300\end{array}$ \\
\hline \multicolumn{9}{|c|}{$\begin{array}{l}\text { Monthly mean discharge, cfs. } \ldots \ldots \ldots \ldots \ldots \ldots \ldots \ldots \ldots \ldots \ldots \ldots \ldots \ldots \ldots \ldots \ldots \ldots \ldots \ldots \ldots \ldots \ldots \ldots \ldots \ldots \ldots \ldots \ldots \ldots \ldots \ldots \\
\text { Runoff, in acre-feet. } \ldots \ldots \ldots \ldots \ldots \ldots \ldots\end{array}$} & $\begin{array}{r}863 \\
53,080\end{array}$ & 7,130 & $\begin{array}{r}90.9 \\
5,590\end{array}$ \\
\hline
\end{tabular}


PLATTE RIVER BASIN

Salt Creek at Lincoln, Nebr. -Continued

Gage height, in feet, and discharge, in cubic feet per second, at indicated time, 1950

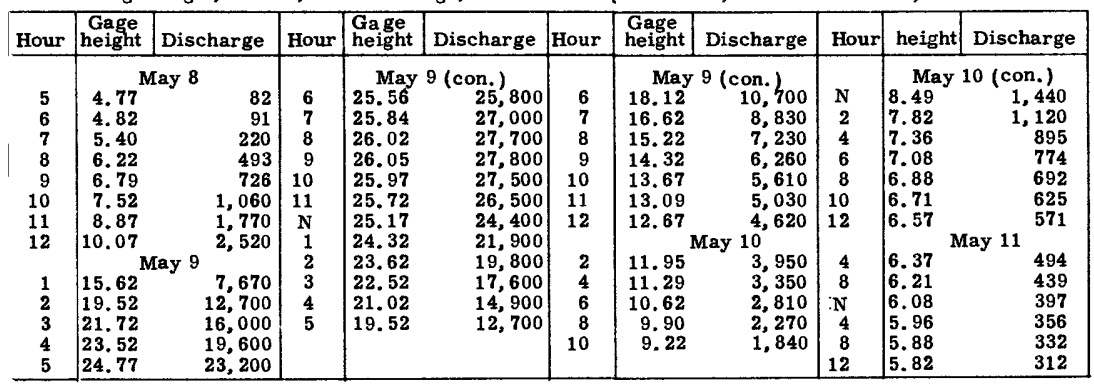

Salt Creek near A shland, Nebr.

Location. --Lat $41^{\circ} 02^{\prime} 50^{\prime \prime}$, long. $96^{\circ} 20^{\prime} 30^{\prime \prime}$, in SW $\frac{1}{4}$ sec. 31, T. $13 \mathrm{~N} .$, R. $10 \mathrm{E}$, at bridge on U. S. Highway 6, 1 mile east of Ashland and $2 \frac{1}{2}$ miles upstream from mouth.

Drainage area. $--1,640 \mathrm{sq} \mathrm{mi}$.

Gage-height record. --Wire-weight gage read twice daily except June 8-21 when no readings were obtained, Mean daily gage heights computed from graph based on gage readings May $5-11,13,18,25,27-29$, June 1, July 10-14, 31 .

Discharge record. --Stage-discharge relation defined by current-meter measurements. Discharge for period of no gage-height record computed on basis of records for station at Lincoln.

Maxima,--May-July 1950: Discharge, 19, 300 cfs 12 p. m. May 9 to 2 a. m. May 10 (gage height, $12.98 \mathrm{ft}$ ).

1947 to April 1950: Discharge observed, 26, $100 \mathrm{cfs}$ May 6, 1949 (gage height, 14. $14 \mathrm{ft}$ ), may include some overflow from Platte River.

Remarks. --Some overflow from Platte River may enter Salt Creek above station when Platte River is in flood.

Mean discharge, in cubic feet per second, 1950

\begin{tabular}{|c|c|c|c|c|c|c|c|c|c|c|c|}
\hline Day & May & June & July & Day & May & June & July & Day & May & June & Iuly \\
\hline $\begin{array}{r}1 \\
\mathbf{2} \\
3 \\
4 \\
5 \\
\mathbf{6} \\
\mathbf{7} \\
8 \\
9 \\
10\end{array}$ & $\begin{array}{r}178 \\
167 \\
156 \\
151 \\
424 \\
606 \\
198 \\
198 \\
12,500 \\
8,660\end{array}$ & $\begin{array}{l}328 \\
244 \\
241 \\
273 \\
219 \\
162 \\
173 \\
150 \\
150 \\
140\end{array}$ & $\begin{array}{r}97 \\
92 \\
97 \\
94 \\
110 \\
110 \\
110 \\
125 \\
173 \\
396\end{array}$ & $\begin{array}{l}11 \\
12 \\
13 \\
14 \\
15 \\
16 \\
17 \\
18 \\
19 \\
20\end{array}$ & $\begin{array}{r}1,000 \\
700 \\
420 \\
307 \\
250 \\
222 \\
184 \\
176 \\
184 \\
257\end{array}$ & $\begin{array}{l}130 \\
130 \\
120 \\
120 \\
820 \\
700 \\
170 \\
230 \\
180 \\
200\end{array}$ & $\begin{array}{r}324 \\
1,130 \\
1,040 \\
420 \\
196 \\
151 \\
361 \\
207 \\
400 \\
706\end{array}$ & $\begin{array}{l}21 \\
22 \\
23 \\
24 \\
25 \\
26 \\
27 \\
28 \\
29 \\
30 \\
31\end{array}$ & $\begin{array}{l}310 \\
254 \\
196 \\
178 \\
488 \\
637 \\
339 \\
216 \\
424 \\
750 \\
556\end{array}$ & $\begin{array}{r}150 \\
140 \\
130 \\
125 \\
122 \\
120 \\
110 \\
105 \\
97 \\
94 \\
- \\
\end{array}$ & $\begin{array}{r}257 \\
151 \\
148 \\
238 \\
257 \\
125 \\
103 \\
110 \\
92 \\
105 \\
426\end{array}$ \\
\hline \multicolumn{9}{|c|}{ 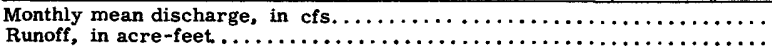 } & $\begin{array}{r}1,009 \\
62,050\end{array}$ & $\begin{array}{r}202 \\
12,050\end{array}$ & $\begin{array}{r}269 \\
16,560\end{array}$ \\
\hline
\end{tabular}

Gage height, in feet, and discharge, in cubic feet per second, at indicated time, 1950

\begin{tabular}{|c|c|c|c|c|c|c|c|c|c|c|c|}
\hline Hour & $\begin{array}{l}\text { Gage } \\
\text { height }\end{array}$ & Discharge & Hour & $\begin{array}{c}\text { Gage } \\
\text { height }\end{array}$ & Discharge & Hour & $\begin{array}{c}\text { Gage } \\
\text { height }\end{array}$ & Discharge & Hour & \begin{tabular}{|l|} 
Gage \\
height
\end{tabular} & Discharge \\
\hline & \multicolumn{2}{|c|}{ May 8} & & \multicolumn{2}{|c|}{ May $9(\mathrm{con})$} & & \multicolumn{2}{|c|}{ May 10 (con } & & \multicolumn{2}{|c|}{ May 11 (con,) } \\
\hline 12 & 2.80 & Lay 9213 & $\begin{array}{l}2 \\
4\end{array}$ & $\begin{array}{l}12.17 \\
12.30\end{array}$ & $\begin{array}{l}15,200 \\
15,800\end{array}$ & $\begin{array}{r}\mathbf{N} \\
2\end{array}$ & $\begin{array}{l}8.09 \\
7.58\end{array}$ & $\begin{array}{l}5,480 \\
4,760\end{array}$ & $\begin{array}{r}6 \\
12\end{array}$ & $\begin{array}{l}4.23 \\
4.14\end{array}$ & $\begin{array}{l}856 \\
802\end{array}$ \\
\hline $\begin{array}{l}1 \\
2 \\
3 \\
4 \\
5\end{array}$ & $\begin{array}{l}2.83 \\
2.92 \\
3.24 \\
5.88 \\
8.75\end{array}$ & $\begin{array}{r}222 \\
250 \\
361 \\
2,540 \\
6,420\end{array}$ & $\begin{array}{r}6 \\
8 \\
10 \\
12\end{array}$ & $\begin{array}{r}12.48 \\
12.68 \\
12.91 \\
12.98\end{array}$ & $\begin{array}{r}16,700 \\
17,700 \\
18,900 \\
19,300 \\
10\end{array}$ & $\begin{array}{r}4 \\
6 \\
8 \\
10 \\
12\end{array}$ & $\begin{array}{l}7.31 \\
6.94 \\
6.49 \\
5.96 \\
5.46\end{array}$ & $\begin{array}{l}4,380 \\
3,870 \\
3,290 \\
2,630 \\
2,040\end{array}$ & $\begin{array}{c}6 a \\
N \\
6 \\
12\end{array}$ & $\begin{array}{l}4.03 \\
3.98 \\
3.91 \\
3.72\end{array}$ & $\begin{array}{r}\text { May } 12 \\
739 \\
711 \\
673 \\
576\end{array}$ \\
\hline 6 & 11.19 & 11,300 & 2 & 12.98 & 19,300 & & & 11 & & & May 13 \\
\hline $\begin{array}{r}7 \\
8 \\
10 \\
N\end{array}$ & $\begin{array}{l}11.58 \\
21.76 \\
11.95 \\
12.08\end{array}$ & $\begin{array}{l}12,600 \\
13,300 \\
14,200 \\
14,800\end{array}$ & $\begin{array}{r}4 \\
6 \\
8 \\
10\end{array}$ & $\begin{array}{r}12.74 \\
12.12 \\
10.57 \\
9.04\end{array}$ & $\begin{array}{r}18,000 \\
15,000 \\
9,570 \\
6,860\end{array}$ & $\begin{array}{r}3 \\
6 \\
9 \\
N\end{array}$ & $\begin{array}{l}4.84 \\
4.42 \\
4.29 \\
4.25\end{array}$ & $\begin{array}{r}1,380 \\
986 \\
894 \\
868\end{array}$ & $\begin{array}{r}6 \\
N \\
6 \\
12\end{array}$ & $\begin{array}{l}3.46 \\
3.33 \\
3.28 \\
3.24\end{array}$ & $\begin{array}{l}451 \\
396 \\
376 \\
361\end{array}$ \\
\hline
\end{tabular}


Wahoo Creek at Ithaca, Nebr.

Location, --Lat $41^{\circ} 08^{\prime} 40^{\prime \prime}$, long. $96^{\circ} 32^{\prime} 20^{\prime \prime}$, in NE corner sec, 32, T. 14 N., R. 8 E. , at bridge on Stat? Highway 63 , one-half mile south of Ithaca.

Drainage area. $-\mathbf{- 2 7 2}$ sq $\mathrm{mi}$.

Gage-height record. - Chain gage read once daily at low stages, more frequently at high stages. No reading obtained May 6.

Discharge record. --Stage-discharge relation defined by current-meter measurements. Shifting-control method used. Discharge for May 6 interpolated.

Maxima. --May-July 1950; Discharge, 1, $310 \mathrm{cfs} 11: 30$ a. m. May 25 (gage height, $15.71 \mathrm{ft}$ ).

October 1949 to April 1950: Discharge, 5, 430 cfs Feb. 28, 1950 (gage height, $21.08 \mathrm{ft}$ ).

Mean discharge, in cubic feet per second, 1950

\begin{tabular}{|c|c|c|c|c|c|c|c|c|c|c|c|}
\hline Day & May & June & July & Day & May & June & July & Day & May & June & July \\
\hline $\begin{array}{r}1 \\
2 \\
3 \\
4 \\
5 \\
6 \\
7 \\
8 \\
9 \\
10\end{array}$ & $\begin{array}{r}30 \\
29 \\
28 \\
29 \\
35 \\
33 \\
31 \\
409 \\
486 \\
58\end{array}$ & $\begin{array}{r}35 \\
26 \\
113 \\
38 \\
30 \\
28 \\
24 \\
20 \\
24 \\
23\end{array}$ & $\begin{array}{l}20 \\
19 \\
20 \\
21 \\
20 \\
.21 \\
19 \\
18 \\
20 \\
26\end{array}$ & $\begin{array}{l}11 \\
12 \\
13 \\
14 \\
15 \\
16 \\
17 \\
18 \\
19 \\
20\end{array}$ & $\begin{array}{l}37 \\
34 \\
34 \\
29 \\
26 \\
28 \\
26 \\
26 \\
24 \\
32\end{array}$ & $\begin{array}{r}23 \\
24 \\
23 \\
37 \\
33 \\
29 \\
34 \\
370 \\
76 \\
34\end{array}$ & $\begin{array}{r}41 \\
159 \\
66 \\
29 \\
26 \\
27 \\
33 \\
64 \\
264 \\
42\end{array}$ & $\begin{array}{l}21 \\
22 \\
23 \\
24 \\
25 \\
26 \\
27 \\
28 \\
29 \\
30 \\
31\end{array}$ & $\begin{array}{r}33 \\
32 \\
24 \\
44 \\
599 \\
60 \\
28 \\
30 \\
415 \\
93 \\
36\end{array}$ & $\begin{array}{l}32 \\
24 \\
21 \\
18 \\
18 \\
17 \\
17 \\
20 \\
17 \\
20 \\
-\end{array}$ & $\begin{array}{l}24 \\
22 \\
22 \\
38 \\
20 \\
18 \\
15 \\
14 \\
18 \\
17 \\
18\end{array}$ \\
\hline \multicolumn{9}{|c|}{$\begin{array}{l}\text { Monthly mean discharge, in cfs. } \ldots \ldots \ldots \ldots \ldots \ldots \ldots \ldots \ldots \ldots \ldots \ldots \ldots \ldots \ldots \ldots \\
\text { Runoff, in acre-feet. } \ldots \ldots \ldots \ldots \ldots \ldots \ldots \ldots \ldots \ldots \ldots \ldots \ldots \ldots \ldots\end{array}$} & $\begin{array}{r}92,2 \\
5,670\end{array}$ & $2,41.6$ & $\begin{array}{r}38.1 \\
2,340\end{array}$ \\
\hline
\end{tabular}

Silver Creek at Ithaca, Nebr.

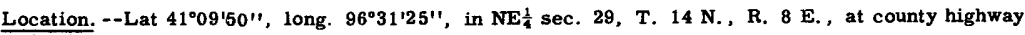
bridge half a mile east of Ithaca.

Drainage area. $-72 \mathrm{sq} \mathrm{mi}$.

Gage-height record. - -Staff-gage read to half-tenths daily.

Discharge record. --Stage-discharge relation defined by current-meter measurements. Shifting-contrcl method used.

Maxima. --May-July 1950: Discharge observed, $28 \mathrm{cfs}$ May 9 (gage height, $3.55 \mathrm{ft}$ ).

1949 to A pril 1950: Discharge, $940 \mathrm{cfs}$ Feb. 28, 1950 (gage height, $10.80 \mathrm{ft}$ ).

Mean discharge, in cubic feet per second, 1950

\begin{tabular}{|c|c|c|c|c|c|c|c|c|c|c|c|}
\hline Day & May & June & July & Day & May & June & July & Day & May & June & July \\
\hline $\begin{array}{r}1 \\
2 \\
3 \\
4 \\
5 \\
6 \\
7 \\
8 \\
9 \\
10\end{array}$ & $\begin{array}{l}5.3 \\
5.3 \\
5.3 \\
5.3 \\
6.4 \\
6.4 \\
6.4 \\
6.4 \\
28 \\
10\end{array}$ & $\begin{array}{l}6.6 \\
6.6 \\
6.6 \\
6.6 \\
5.5 \\
5.5 \\
4.4 \\
4.4 \\
4.4 \\
4.4\end{array}$ & $\begin{array}{l}4.4 \\
4.4 \\
4.4 \\
4.4 \\
4.4 \\
4.4 \\
4.4 \\
4.4 \\
4.4 \\
4.4\end{array}$ & $\begin{array}{l}11 \\
12 \\
13 \\
14 \\
15 \\
16 \\
17 \\
18 \\
19 \\
20\end{array}$ & $\begin{array}{l}8.2 \\
8.2 \\
7.0 \\
7.0 \\
7.0 \\
7.0 \\
5.9 \\
5.9 \\
8.2 \\
6.8\end{array}$ & $\begin{array}{l}4.4 \\
4.4 \\
4.4 \\
4.4 \\
4.4 \\
5.5 \\
6.6 \\
5.5 \\
5.5 \\
4.4\end{array}$ & $\begin{array}{c}4.4 \\
4.4 \\
4.3 \\
4.3 \\
4.3 \\
11 \\
9.8 \\
6.4 \\
5.3 \\
5.3\end{array}$ & $\begin{array}{l}21 \\
22 \\
23 \\
24 \\
25 \\
26 \\
27 \\
28 \\
29 \\
30 \\
31 \\
\end{array}$ & $\begin{array}{c}6.8 \\
6.8 \\
6.8 \\
6.8 \\
13 \\
10 . \\
7.7 \\
7.7 \\
6.6 \\
6.6 \\
6.6 \\
\end{array}$ & $\begin{array}{l}4.4 \\
4.4 \\
4.4 \\
4.4 \\
4.4 \\
4.4 \\
4.4 \\
4.4 \\
4.4 \\
4.4 \\
-\end{array}$ & $\begin{array}{l}5.0 \\
5.0 \\
5.0 \\
5.0 \\
5.0 \\
5.0 \\
4.1 \\
4.1 \\
4.1 \\
3.9 \\
3.9\end{array}$ \\
\hline \multicolumn{9}{|c|}{ 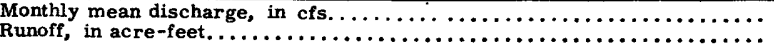 } & $\begin{array}{r}7.79 \\
479 \\
\end{array}$ & $\begin{array}{r}4.95 \\
295 \\
\end{array}$ & $\begin{array}{r}4.95 \\
305 \\
\end{array}$ \\
\hline
\end{tabular}




\section{Weeping Water Creek basin}

Weeping Water Creek at Union, Nebr

Location. --Lat $40^{\circ} 48^{\prime} 50^{\prime \prime}$ ', long. $95^{\circ} 55^{\prime} 45^{\prime \prime}$, in NW/ sec. 26, T. $10 \mathrm{~N} ., \mathrm{R} .13 \mathrm{E}$., at bridge on U. S. Highway 34, a quarter of a mile west of Union and three-quarters of a mile downstream from South Branch Weeping Water Creek.

Drainage area. $--231 \mathrm{sq} \mathrm{mL}$.

Gage-height record. --Chain gage read once daily prior to June 28 and wire-weight gage read once daily thereafter; gages read frequently during rises. Gage observer's house destroyed by flood on May 9 and record for periods May 1-3, 8, lost; no readings by observer May 9-18. Gage-height record for period May 8-11 computed from graph based on engineers' gage readings, floodmark, and observer's notes.

Discharge record. --Stage-discharge relation defined by current-meter measurements below $11,000 \mathrm{cfs}$ and extended to peak stage on basis of computation of combined nlow through bridges and over highway embankment. Shifting-control method used May 19 to July 11, .Tuly 14-30. Discharge for periods of no gage-height record May 1-3, 11-18, computed on basis of discharge measurement of May 17, weather records, and trend of flow.

Maxima, --May-July 1950: Discharge, 60, 300 cfs 12:30 a. m. May 9 (gage height, 29. B ft, from floodmark), by computation of combined flow through bridges and over highway embankment.

February to April 1950: Discharge, 1, $300 \mathrm{cfs}$ Feb. 28 (gage height, 15. 15 ft).

Mean discharge, in cubic feet per second, 1950

\begin{tabular}{|c|c|c|c|c|c|c|c|c|c|c|c|}
\hline Day & May & June & July & Day & May & June & July & Day & May & June & July \\
\hline $\begin{array}{r}1 \\
2 \\
3 \\
4 \\
5 \\
6 \\
7 \\
8 \\
9 \\
10\end{array}$ & $\begin{array}{r}15 \\
15 \\
20 \\
286 \\
2,080 \\
146 \\
44 \\
7,080 \\
16,900 \\
487\end{array}$ & $\begin{array}{r}32 \\
28 \\
26 \\
21 \\
13 \\
11 \\
16 \\
17 \\
224 \\
92\end{array}$ & $\begin{array}{c}12 \\
11 \\
9.5 \\
11 \\
10 \\
9.2 \\
7.7 \\
20 \\
27\end{array}$ & $\begin{array}{l}12 \\
13 \\
14 \\
15 \\
16 \\
17 \\
18 \\
19 \\
20\end{array}$ & $\begin{array}{r}200 \\
70 \\
50 \\
50 \\
40 \\
40 \\
42 \\
53 \\
64 \\
62\end{array}$ & $\begin{array}{r}16 \\
15 \\
20 \\
844 \\
132 \\
16 \\
432 \\
190 \\
37 \\
30\end{array}$ & $\begin{array}{r}60 \\
1,960 \\
700 \\
190 \\
107 \\
11 \\
8.6 \\
94 \\
49 \\
35\end{array}$ & $\begin{array}{l}23 \\
24 \\
25 \\
26 \\
27 \\
28 \\
29 \\
30 \\
31\end{array}$ & $\begin{array}{l}82 \\
43 \\
31 \\
27 \\
32 \\
41 \\
32 \\
28 \\
73 \\
43 \\
40\end{array}$ & $\begin{array}{r}19 \\
15 \\
51 \\
1,140 \\
85 \\
26 \\
13 \\
14 \\
16 \\
12 \\
-\end{array}$ & $\begin{array}{c}28 \\
26 \\
26 \\
24 \\
22 \\
19 \\
17 \\
14 \\
13 \\
8.0 \\
1,230 \\
\end{array}$ \\
\hline \multicolumn{9}{|c|}{$\begin{array}{l}\text { Monthiy mean discharge, in cfs } \ldots \ldots \ldots \ldots \ldots \ldots \ldots \ldots \ldots \ldots \ldots \ldots \ldots \ldots \ldots \ldots \\
\text { Runoff, in acre-feet. } \ldots \ldots \ldots \ldots \ldots \ldots \ldots \ldots \ldots \ldots \ldots \ldots \ldots \ldots \ldots\end{array}$} & $\begin{array}{r}910 \\
55,970\end{array}$ & 7,150 & $\begin{array}{r}154 \\
9,460\end{array}$ \\
\hline
\end{tabular}

Gage height, in feet, and discharge, in cubic feet per second, at indicated time, 1950

\begin{tabular}{|c|c|c|c|c|c|c|c|c|c|c|c|}
\hline Hour & $\begin{array}{l}\text { Gage } \\
\text { height }\end{array}$ & Discharge & Hour & $\begin{array}{c}\text { Gage } \\
\text { height }\end{array}$ & Discharge & Hour & $\begin{array}{c}\text { Gage } \\
\text { height }\end{array}$ & Discharge & Hour & $\begin{array}{c}\text { Gage } \\
\text { height }\end{array}$ & Discharge \\
\hline $\begin{array}{r}5 \\
6 \\
7 \\
8 \\
9 \\
10 \\
11 \\
12\end{array}$ & $\begin{array}{l}6.46 \\
7.05 \\
13.65 \\
21.65 \\
26.75 \\
28.25 \\
29.12 \\
29.70\end{array}$ & $\begin{array}{r}\text { ay } 8 \\
36 \\
72 \\
933 \\
3,680 \\
34,000 \\
46,600 \\
54,200 \\
59,400\end{array}$ & $\begin{array}{c}12: 30 \\
1 \\
2 \\
3 \\
4 \\
5 \\
6 \\
7 \\
8 \\
9 \\
10 \\
11\end{array}$ & $\begin{array}{l}29.80 \\
29.60 \\
28.65 \\
27.50 \\
26.40 \\
25.75 \\
25.40 \\
25.25 \\
25.05 \\
24.85 \\
24.60 \\
24.40\end{array}$ & $\begin{array}{l}9 \\
60,300 \\
58,500 \\
50,000 \\
40,200 \\
31,200 \\
26,000 \\
23,200 \\
22,000 \\
20,400 \\
18,800 \\
16,800 \\
15,200\end{array}$ & $\begin{array}{r}\mathbf{N} \\
1 \\
2 \\
3 \\
4 \\
6 \\
8 \\
10 \\
12 \\
\\
2 \\
4\end{array}$ & $\begin{array}{r}\text { May } \\
23.95 \\
23.40 \\
23.00 \\
22.60 \\
22.15 \\
20.85 \\
18.50 \\
16.90 \\
15.50 \\
M \\
14.35 \\
13.30\end{array}$ & $\begin{array}{r}9 \text { (con.) } \\
11,600 \\
7,700 \\
6,000 \\
4,960 \\
4,180 \\
3,200 \\
2,330 \\
1,820 \\
1,400 \\
10 \\
1,090 \\
860\end{array}$ & $\begin{array}{r}6 \\
8 \\
10 \\
N \\
2 \\
4 \\
6 \\
9 \\
12\end{array}$ & $\begin{array}{l}\text { May } \\
12.40 \\
11.55 \\
10.80 \\
10.20 \\
9.70 \\
9.30 \\
8.90 \\
8.45 \\
8.10\end{array}$ & 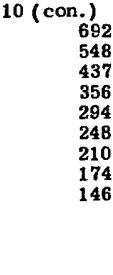 \\
\hline
\end{tabular}




\section{Little Nemaha River basin}

Little Nemaha River at Auburn, Nebr.

Location. --Lat $40^{\circ} 23^{\prime} 30^{\prime \prime}$, long. $95^{\circ} 48^{\prime} 40^{\prime \prime}$, in NW $\frac{1}{4}$ sec. 23, T. 5 N., R. 14 E., at bridge on State Highway 3, 1 mile downstream from Longs Creek and 1 mile east of Auburn.

Drainage area. --664 sq mi.

Gage-height record. --Water-stage recorder graph above $9 \mathrm{ft}$ except $11 \mathrm{p}$. $\mathrm{m}$. . May 8 to $5 \mathrm{a} . \mathrm{m}$. May 10 when recorder failed to operate. Twice daily wire-weight gage readings below 9 ft except May 11-13, $16,18,20,28$, June 1, 4, 5, 7, 12, 22, 25, 27, July 2, 5, 7, 14, 16, 19, 20, 22-24, 26, 27, 30, when no readings were obtained.

Discharge record. --Stage-discharge relation defined by current-meter measurements below 12, 000 cfs and extended to peak stage on basis of computation of combined flow through bridges and culverts and over highway and railway embankments. Shifting-control method used. Discharge for periods of no gage-height records was computed on the basis of floodmark on May 9, weather records, and trend of flow, or was interpolated.

Maxima, --May-July 1950: Discharge, 164, $000 \mathrm{cfs} 7 \mathrm{a}, \mathrm{m}$. May 9 (gage height, 27. $65 \mathrm{ft}$, from floodmark). August 1949 to April 1950: Discharge, 2, 080 cfs Sept. 6, 1949 (gage height, $10.73 \mathrm{ft}$ ).

Mean discharge, in cubic feet per second, 1950

\begin{tabular}{|c|c|c|c|c|c|c|c|c|c|c|c|}
\hline Day & May & June & July & Day & May & June & July & Day & May & June & July \\
\hline $\begin{array}{r}1 \\
2 \\
3 \\
4 \\
5 \\
6 \\
7 \\
8 \\
9 \\
10\end{array}$ & $\begin{array}{r}56 \\
55 \\
55 \\
55 \\
4,940 \\
523 \\
158 \\
777 \\
44,900 \\
2,300\end{array}$ & $\begin{array}{r}142 \\
112 \\
104 \\
97 \\
91 \\
87 \\
84 \\
75 \\
2,780 \\
338\end{array}$ & $\begin{array}{r}96 \\
73 \\
55 \\
48 \\
47 \\
46 \\
44 \\
42 \\
110 \\
146\end{array}$ & $\begin{array}{l}11 \\
12 \\
13 \\
14 \\
15 \\
16 \\
17 \\
18 \\
19 \\
20\end{array}$ & $\begin{array}{l}740 \\
450 \\
300 \\
226 \\
199 \\
172 \\
146 \\
145 \\
145 \\
210\end{array}$ & $\begin{array}{r}118 \\
100 \\
87 \\
1,010 \\
706 \\
150 \\
1,950 \\
948 \\
197 \\
120\end{array}$ & $\begin{array}{r}100 \\
4,060 \\
1,130 \\
460 \\
170 \\
178 \\
183 \\
250 \\
182 \\
145\end{array}$ & $\begin{array}{l}21 \\
22 \\
23 \\
24 \\
25 \\
26 \\
27 \\
28 \\
29 \\
30 \\
31\end{array}$ & $\begin{array}{r}292 \\
200 \\
130 \\
91 \\
1,670 \\
357 \\
231 \\
540 \\
1,220 \\
1,420 \\
181\end{array}$ & $\begin{array}{l}95 \\
69 \\
83 \\
79 \\
71 \\
65 \\
59 \\
55 \\
52 \\
55 \\
-\end{array}$ & $\begin{array}{r}100 \\
96 \\
92 \\
88 \\
85 \\
80 \\
74 \\
69 \\
52 \\
118 \\
248\end{array}$ \\
\hline $\begin{array}{l}\text { Mo } \\
\text { Rur }\end{array}$ & near & arge & & & & & & $\cdots$ & $\begin{array}{r}2,029 \\
124,700\end{array}$ & $\begin{array}{r}333 \\
19,830\end{array}$ & $\begin{array}{r}280 \\
17,190\end{array}$ \\
\hline
\end{tabular}

Gage height, in feet, and discharge, in cubic feet per second, at indicated time, 1950

\begin{tabular}{|c|c|c|c|c|c|c|c|c|c|c|c|}
\hline Hour & $\begin{array}{l}\text { Gage } \\
\text { height }\end{array}$ & Discharge & Hour & $\begin{array}{c}\text { Gage } \\
\text { height }\end{array}$ & Discharge & Hour & $\begin{array}{r}\text { Gage } \\
\text { height }\end{array}$ & Discharge & Hour & $\begin{array}{l}\text { Gage } \\
\text { height }\end{array}$ & Discharge \\
\hline $\begin{array}{c}7 p \\
8 \\
9 \\
10 \\
11 \\
12\end{array}$ & \begin{tabular}{|l} 
\\
$5.70^{N}$ \\
5.72 \\
5.76 \\
5.85 \\
16.35 \\
18.10 \\
19.72 \\
21.29 \\
22.94 \\
24.68 \\
26.03 \\
27.31 \\
\end{tabular} & $\begin{array}{rr}\text { May } 8 & 150 \\
154 \\
162 \\
181 \\
9,090 \\
12,200 \\
\text { May } 9 \\
15,800 \\
20,500 \\
27,200 \\
45,400 \\
80,900 \\
140,000\end{array}$ & $\begin{array}{r}7 \\
8 \\
9 \\
10 \\
11 \\
\mathrm{~N} \\
1 \\
2 \\
3 \\
4 \\
5 \\
6\end{array}$ & $\begin{array}{l}\text { May } \\
27.65 \\
27.50 \\
26.88 \\
24.99 \\
24.99 \\
23.82 \\
22.70 \\
21.62 \\
20.48 \\
19.26 \\
18.13 \\
17.11\end{array}$ & $\begin{array}{r}9 \text { (con.) } \\
164,000 \\
153,000 \\
116,000 \\
80,600 \\
52,700 \\
33,300 \\
26,000 \\
21,600 \\
18,000 \\
14,600 \\
12,300 \\
10,300\end{array}$ & \begin{tabular}{|r}
7 \\
8 \\
9 \\
10 \\
11 \\
12 \\
\\
1 \\
2 \\
3 \\
4 \\
5 \\
6
\end{tabular} & $\begin{array}{l}\text { May } \\
15.96 \\
15.52 \\
15.12 \\
14.71 \\
14.30 \\
13.90 \\
13.47 \\
13.10 \\
12.70 \\
12.30 \\
11.90 \\
11.49\end{array}$ & 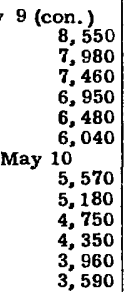 & $\begin{array}{r}7 \\
8 \\
9 \\
10 \\
11 \\
\mathrm{~N} \\
2 \\
4 \\
6 \\
8 \\
10 \\
12\end{array}$ & \begin{tabular}{|r|} 
May \\
11.09 \\
10.74 \\
10.45 \\
10.11 \\
9.80 \\
9.53 \\
9.15 \\
8.86 \\
8.62 \\
8.42 \\
8.25 \\
8.12
\end{tabular} & $\begin{array}{r}0 \text { (con.) } \\
3,230 \\
2,650 \\
2,340 \\
1,960 \\
1,600 \\
1,220 \\
1,140 \\
1,060 \\
1,030 \\
972 \\
935 \\
920\end{array}$ \\
\hline
\end{tabular}


Nemahá River basin

Nemaha River at Falls City, Nebr.

Location. --Lat $40^{\circ} 02^{\prime} 00^{\prime \prime}$, long. $95^{\circ} 35^{\prime} 30^{\prime \prime}$, on line between secs. 22 and 23, T. 1 N., R. 16 E., at bridge, on,U. S. Highway 73, 1 mile south of Falls City and about 13 miles upstream from mouth. Datum of gage is $861.24 \mathrm{ft}$ above mean sea level, datum of 1929 , supplementary adjustment of 1943 , tentative (levels by Corps of Engineers).

Drainage.area. $--1,340 \mathrm{sq}$ mi.

Gage-height record.--Wire-weight gage read twice daily. Gage heights for the following periods were obtained from graph based on gage readings: May 5-12, 21-26, May 28 to June 1, June 10, July 2, 9-15, 17-23.

Discharge .record. --Stage-discharge relation defined by current-meter measurements. Shifting-control method used.

Maxima. --May-July 1950: Discharge, 26, 300 cfs 10:30 p. m. May 9 (gage height, 26. 0 ft). 1944' to April 1950: Discharge, 34, 200 cfs June 2, 1949 (gage height, $28.8 \mathrm{ft}$, from floodmark).

Mean discharge, in cubic feet per second, 1950

\begin{tabular}{|c|c|c|c|c|c|c|c|c|c|c|c|}
\hline Day & May & June & July & Day & May & June & July & Day & May & June & July \\
\hline $\begin{array}{r}1 \\
2 \\
3 \\
4 \\
5 \\
6 \\
7 \\
8 \\
9 \\
10\end{array}$ & $\begin{array}{r}80 \\
82 \\
72 \\
74 \\
1,330 \\
506 \\
805 \\
390 \\
16,400 \\
6,360\end{array}$ & $\begin{array}{l}821 \\
496 \\
447 \\
404 \\
335 \\
230 \\
208 \\
213 \\
338 \\
760\end{array}$ & $\begin{array}{r}72 \\
233 \\
164 \\
159 \\
130 \\
83 \\
83 \\
83 \\
784 \\
1,200\end{array}$ & $\begin{array}{l}11 \\
12 \\
13 \\
14 \\
15 \\
16 \\
17 \\
18 \\
19 \\
20\end{array}$ & $\begin{array}{r}1,590 \\
660 \\
461 \\
332 \\
284 \\
242 \\
197 \\
157 \\
197 \\
181\end{array}$ & $\begin{array}{l}329 \\
239 \\
171 \\
157 \\
143 \\
152 \\
760 \\
980 \\
350 \\
308\end{array}$ & $\begin{array}{r}428 \\
420 \\
2,060 \\
380 \\
404 \\
242 \\
1,400 \\
2,880 \\
828 \\
618\end{array}$ & $\begin{array}{l}21 \\
22 \\
23 \\
24 \\
25 \\
26 \\
27 \\
28 \\
29 \\
30 \\
31 \\
\end{array}$ & $\begin{array}{r}2,170 \\
2,440 \\
695 \\
296 \\
2,990 \\
2,110 \\
1,240 \\
660 \\
4,060 \\
3,980 \\
2,060 \\
\end{array}$ & $\begin{array}{r}239 \\
205 \\
134 \\
118 \\
118 \\
98 \\
88 \\
81 \\
76 \\
62 \\
- \\
\end{array}$ & $\begin{array}{l}835 \\
468 \\
227 \\
166 \\
166 \\
169 \\
230 \\
148 \\
128 \\
118 \\
106 \\
\end{array}$ \\
\hline \multicolumn{9}{|c|}{$\begin{array}{l}\text { Monthly mean discharge, in cfs. } \ldots \ldots \ldots \ldots \ldots \ldots \ldots \ldots \ldots \ldots \ldots \ldots \ldots \ldots \ldots \ldots \ldots \ldots \\
\text { Runoff, in acre-feet. } \ldots \ldots \ldots \ldots \ldots \ldots \ldots \ldots \ldots \ldots \ldots \ldots \ldots \ldots \ldots\end{array}$} & $\begin{array}{r}1,713 \\
105,300 \\
\end{array}$ & 17,870 & $\begin{array}{r}497 \\
30,570\end{array}$ \\
\hline
\end{tabular}

Gage height, in feet, and discharge, in cubic feet per second, at indicated time, 1950

\begin{tabular}{|c|c|c|c|c|c|c|c|c|c|c|c|}
\hline Hour & $\begin{array}{c}\text { Gage } \\
\text { height }\end{array}$ & Discharge & Hour & $\begin{array}{r}\text { Gage } \\
\text { height }\end{array}$ & Discharge & Hour & $\begin{array}{c}\text { Gage } \\
\text { height }\end{array}$ & Discharge & Hour & $\begin{array}{l}\text { Gage } \\
\text { height }\end{array}$ & Discharge \\
\hline $\begin{array}{c}8 p \\
10 \\
12 \\
1 \\
2 \\
3 \\
4 \\
5 \\
6 \\
7 \\
8 \\
9 \\
10\end{array}$ & \begin{tabular}{|c}
4.50 \\
4.50 \\
4.59 \\
8.00 \\
10.00 \\
12.55 \\
14.62 \\
16.30 \\
17.30 \\
18.05 \\
18.66 \\
19.22 \\
19.90 \\
20.53
\end{tabular} & $\begin{array}{rr}\text { ay } 8 & 262 \\
& 294 \\
& 2,280 \\
9 & \\
3,800 \\
6,000 \\
8,080 \\
9,930 \\
11,000 \\
11,900 \\
12,600 \\
13,300 \\
14,200 \\
15,000\end{array}$ & $\begin{array}{c}11 \\
\mathrm{~N} \\
1 \\
2 \\
3 \\
4 \\
5 \\
6 \\
7 \\
8 \\
9 \\
10 \\
10: 30 \\
11\end{array}$ & $\begin{array}{l}\text { May } \\
21.20 \\
21.78 \\
22.15 \\
22.50 \\
22.88 \\
23.33 \\
23.80 \\
24.30 \\
24.77 \\
25.23 \\
25.70 \\
25.95 \\
26.00 \\
25.97\end{array}$ & $\begin{array}{r}9 \text { (con.) } \\
15,900 \\
16,900 \\
17,600 \\
18,300 \\
19,100 \\
20,000 \\
20,900 \\
22,000 \\
23,200 \\
24,400 \\
25,600 \\
26,200 \\
26,300 \\
26,200\end{array}$ & $\begin{array}{r}1 \\
2 \\
3 \\
4 \\
5 \\
6 \\
8 \\
10 \\
N \\
2 \\
4 \\
6 \\
8\end{array}$ & $\begin{array}{l}20.87 \\
18.20 \\
16.85 \\
15.60 \\
14.78 \\
14.08 \\
13.22 \\
12.73 \\
12.30 \\
11.91 \\
11.42 \\
11.00 \\
10.55 \\
10.10\end{array}$ & $\begin{array}{r}10 \\
15,400 \\
11,800 \\
10,200 \\
8,620 \\
7,690 \\
6,930 \\
6,070 \\
5,810 \\
5,220 \\
4,870 \\
4,430 \\
4,050 \\
3,670 \\
3,310 \\
2,960\end{array}$ & $\begin{array}{r}2 \\
4 \\
6 \\
8 \\
10 \\
N \\
4 \\
8 \\
12\end{array}$ & $\begin{array}{l}9.19 \\
8.73 \\
8.30 \\
7.95 \\
7.67 \\
7.40 \\
6.93 \\
6.58 \\
6.34\end{array}$ & $\begin{array}{r}11 \\
2,630 \\
2,310 \\
2,010 \\
1,770 \\
1,600 \\
1,440 \\
1,160 \\
970 \\
850\end{array}$ \\
\hline
\end{tabular}

\section{Kansas River basin}

Republican River near Bloomington, Nebr.

Location, --Lat $40^{\circ} 04^{\prime} 00^{\prime \prime}$, long. $99^{\circ} 02^{\prime} 05^{\prime \prime}$, in NW $\frac{1}{4} \mathrm{SE} \frac{1}{\mathrm{~s}}$ sec. 8, T, 1 N., R. $15 \mathrm{~W}$. $600 \mathrm{ft}$ downstream from county highway bridge, 2 miles south of Bloomington, and $9 \frac{1}{2}$ miles downstream from Turkey Creek. Datum of gage is $1,824.15 \mathrm{ft}$ above mean sea level, datum of 1929 .

Drainage area. $--20,800 \mathrm{sq} \mathrm{mi}$, of which only $15,100 \mathrm{sq}$ mi contribute directly to surface runoff.

Gage-height record. --Water-stage recorder graph except July 8-12, when range of stage only was recorded.

Discharge record. --Stage-discharge relation defined by current-meter measurements. Shifting-control method used. Discharge for period July 8-12 computed on basis of recorded range in stage and records for station near Orleans.

Maxima.--May-July 1950: Discharge, 5, $050 \mathrm{cfs} 9$ a. m. July 28 (gage height, 5. $66 \mathrm{ft}$ ).

1929 to April 1950: Discharge, 260,000 cfs June 1, 1935 (gage height, 20.4 ft, from floodmarks, site then in use), by slope-area method.

Remarks. - Natural flow affected by irrigation development above station. 
Republican River near Bloomington, Nebr. -Continued

Mean discharge, in cubic feet per second, 1950

\begin{tabular}{|c|c|c|c|c|c|c|c|c|c|c|c|}
\hline Day & May & June & July & Day & May & June & July & Day & May & June & July \\
\hline $\begin{array}{r}1 \\
2 \\
3 \\
4 \\
5 \\
6 \\
7 \\
8 \\
9 \\
10\end{array}$ & $\begin{array}{l}372 \\
363 \\
363 \\
363 \\
396 \\
405 \\
421 \\
396 \\
396 \\
890\end{array}$ & $\begin{array}{r}\mathbf{1} \mathbf{4 5 0} \\
\mathbf{9 5 7} \\
\mathbf{7 8 6} \\
\mathbf{6 7 2} \\
\mathbf{5 7 0} \\
\mathbf{5 0 6} \\
\mathbf{4 2 6} \\
\mathbf{3 6 7} \\
\mathbf{3 2 7} \\
\mathbf{2 9 4}\end{array}$ & $\begin{array}{r}68 \\
84 \\
130 \\
153 \\
189 \\
908 \\
922 \\
600 \\
350 \\
300\end{array}$ & $\begin{array}{l}11 \\
12 \\
13 \\
14 \\
15 \\
16 \\
17 \\
18 \\
19 \\
20\end{array}$ & $\begin{array}{r}887 \\
852 \\
929 \\
1,190 \\
798 \\
636 \\
541 \\
512 \\
898 \\
1,750\end{array}$ & $\begin{array}{l}267 \\
237 \\
213 \\
193 \\
181 \\
171 \\
159 \\
325 \\
452 \\
278\end{array}$ & $\begin{array}{r}300 \\
350 \\
666 \\
1,920 \\
1,710 \\
1,200 \\
660 \\
382 \\
294 \\
684\end{array}$ & $\begin{array}{l}21 \\
22 \\
23 \\
24 \\
25 \\
26 \\
27 \\
28 \\
29 \\
30 \\
31\end{array}$ & $\begin{array}{r}1,030 \\
810 \\
660 \\
512 \\
436 \\
405 \\
410 \\
848 \\
2,720 \\
3,620 \\
2,520 \\
\end{array}$ & $\begin{array}{r}227 \\
193 \\
168 \\
150 \\
127 \\
106 \\
96 \\
86 \\
73 \\
70 \\
- \\
\end{array}$ & $\begin{array}{r}901 \\
594 \\
512 \\
1,370 \\
1,220 \\
1,830 \\
3,520 \\
4,940 \\
4,530 \\
3,680 \\
2,470 \\
\end{array}$ \\
\hline \multicolumn{9}{|c|}{ 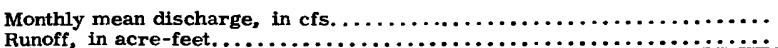 } & $\begin{array}{r}882 \\
54,210 \\
\end{array}$ & $\begin{array}{r}338 \\
20,090 \\
\end{array}$ & $\begin{array}{r}1,208 \\
74,260 \\
\end{array}$ \\
\hline
\end{tabular}

Republican River near Hardy, Nebr.

Location. --Lat $40^{\circ} 00^{\prime}$, long. $97^{\circ} 56^{\prime}$, in sec. 6, T. 1 S., R. 5 W., $1 \frac{1}{4}$ miles southwest of Hardy. Datum of gage is $1,501.46 \mathrm{ft}$ above mean sea level, datum of 1929 .

Drainage area. $--22,400 \mathrm{sq} \mathrm{mi}$, of which $1,900 \mathrm{sq} \mathrm{mi}$ is largely noncontributing.

Gage-height record. --Water-stage recorder graph except for periods May 19-30, June 2-15, 19-27, July 8, 20-24, for which graph was based on twice-daily gage readings, and period June 18-18, for which there is no gage-beight record. $\frac{\text { Discharge record. }}{\text { method used. }}$-Stage-discharge relation defined by current-meter measurements. Shirting-control

Maxima, --May-July 1950: Discharge, 30, 000 cfs 2 a. m. July 10 (gage height, $13.70 \mathrm{ft}$ ).

1932 to April 1950: Discharge, 225,000 cfs June 2, 1935 (gage height, 19.4 ft), by slope-area method.

Remarks. --Natural flow affected by irrigation developments above station.

Mean discharge, in cubic feet per second, 1950

\begin{tabular}{|c|c|c|c|c|c|c|c|c|c|c|c|}
\hline Day & May & June & July & Day & May & June & July & Day & May & June & July \\
\hline $\begin{array}{r}1 \\
2 \\
3 \\
4 \\
5 \\
6 \\
7 \\
8 \\
9 \\
10\end{array}$ & $\begin{array}{l}477 \\
442 \\
456 \\
456 \\
540 \\
556 \\
526 \\
540 \\
755 \\
665\end{array}$ & $\begin{array}{r}2,840 \\
1,940 \\
1,190 \\
980 \\
863 \\
746 \\
665 \\
540 \\
465 \\
358\end{array}$ & $\begin{array}{r}168 \\
170 \\
178 \\
178 \\
182 \\
168 \\
205 \\
707 \\
12,800 \\
12,600\end{array}$ & $\begin{array}{l}11 \\
12 \\
13 \\
14 \\
15 \\
16 \\
17 \\
18 \\
19 \\
20\end{array}$ & $\begin{array}{r}556 \\
953 \\
989 \\
971 \\
1,020 \\
1,280 \\
980 \\
845 \\
863 \\
881\end{array}$ & $\begin{array}{l}340 \\
330 \\
325 \\
290 \\
290 \\
285 \\
275 \\
260 \\
254 \\
244\end{array}$ & $\begin{array}{r}2,070 \\
1,260 \\
821 \\
740 \\
905 \\
2,100 \\
8,220 \\
3,000 \\
1,250 \\
845\end{array}$ & $\begin{array}{l}21 \\
22 \\
23 \\
24 \\
25 \\
26 \\
27 \\
28 \\
29 \\
30 \\
31\end{array}$ & $\begin{array}{r}1,640 \\
1,940 \\
1,220 \\
1,020 \\
890 \\
728 \\
665 \\
647 \\
1,370 \\
2,920 \\
3,640 \\
\end{array}$ & $\begin{array}{l}463 \\
340 \\
295 \\
241 \\
210 \\
202 \\
200 \\
190 \\
180 \\
175 \\
- \\
\end{array}$ & $\begin{array}{r}701 \\
809 \\
935 \\
872 \\
854 \\
1,520 \\
1,260 \\
3,920 \\
5,220 \\
4,540 \\
\mathbf{3}, 990 \\
\end{array}$ \\
\hline \multicolumn{9}{|c|}{ 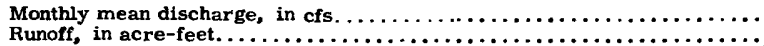 } & $\begin{array}{r}1,014 \\
62,340\end{array}$ & $\begin{array}{r}532 \\
31,670\end{array}$ & $\begin{array}{r}2,362 \\
145,200\end{array}$ \\
\hline
\end{tabular}

Gage height, in feet, and discharge, in cubic feet per second, at indicated time, 1950

\begin{tabular}{|c|c|c|c|c|c|c|c|c|c|c|c|}
\hline Hour & $\begin{array}{c}\text { Gage } \\
\text { height }\end{array}$ & Discharge & Hour & $\begin{array}{c}\text { Gage } \\
\text { height }\end{array}$ & Discharge & Hour & $\begin{array}{c}\text { Gage } \\
\text { height }\end{array}$ & Discharge & Hour & $\begin{array}{c}\text { Gage } \\
\text { height }\end{array}$ & Discharge \\
\hline $\begin{array}{r}4 \\
8 \\
N \\
4 \\
8 \\
12 \\
\\
4 \\
8 \\
\mathbf{N}\end{array}$ & $\begin{array}{r}4.30 \\
4.65 \\
5.10 \\
5.15 \\
5.15 \\
5.40 \\
6.00 \\
9.90 \\
10.90\end{array}$ & $\begin{array}{r} \\
290 \\
505 \\
890 \\
935 \\
935 \\
1,160 \\
9 \\
1,820 \\
9,800 \\
13,000\end{array}$ & $\begin{array}{r}4 \\
8 \\
12 \\
2 \\
2 . \\
4 \\
8 \\
\mathbf{N} \\
4 \\
8 \\
12\end{array}$ & $\begin{array}{r}\text { July } 9 \\
11.70 \\
12.50 \\
13.45 \\
13.70 \\
13.45 \\
11.15 \\
9.15 \\
7.77 \\
6.80 \\
6.20\end{array}$ & $\begin{array}{r}\text { (Con.) } \\
16,100 \\
20,400 \\
27,300 \\
\text { July } 10 \\
30,000 \\
27,300 \\
14,100 \\
8,400 \\
5,500 \\
3,900 \\
3,070\end{array}$ & $\begin{array}{r}4 \\
8 \\
\mathbf{N} \\
4 \\
8 \\
12 \\
4 \\
4 \\
\mathbf{8}\end{array}$ & $\begin{array}{l}5.70 \\
5.40 \\
5.15 \\
5.05 \\
4.92 \\
4.82 \\
4.70 \\
4.57 \\
4.45\end{array}$ & $\begin{array}{r}\text { uly } 11 \\
2,450 \\
2,100 \\
1,870 \\
1,780 \\
1,660 \\
1,570 \\
12 \\
1,460 \\
1,350 \\
1,260\end{array}$ & $\begin{array}{r}4 \\
8 \\
12 \\
4 \\
8 \\
\mathbf{N} \\
4 \\
8 \\
12\end{array}$ & $\begin{array}{l}\text { July } \\
4.32 \\
4.15 \\
4.10 \\
4.00 \\
3.91 \\
3.84 \\
3.73 \\
3.72 \\
3.67\end{array}$ & $\begin{array}{r}12 \text { (Con.) } \\
1,150 \\
1,030 \\
990 \\
\text { July } 13 \\
\\
920 \\
855 \\
810 \\
740 \\
730 \\
700\end{array}$ \\
\hline
\end{tabular}


Center Creek at Franklin, Nebr.

Location. --Lat $40^{\circ} 05^{\prime} 30^{\prime \prime}$, long. $98^{\circ} 57^{\prime} 50^{\prime \prime}$, in SE $\frac{1}{4} \mathrm{SW} \frac{1}{4}$ sec. 36, T. 2 N., R. 15 W., at bridge on State Highway 3 at Franklin, $1 \frac{1}{4}$ miles upstream from mouth. Datum of gage is $1,828.07 \mathrm{ft}$ above mean sea level, datum of 1929 .

Drainage area. $--111 \mathrm{sq} \mathrm{mi}$.

Gage-height record. --Staff gage read twice daily.

Discharge record. --Stage-discharge relation defined by current-meter measurements. Shifting-control method used.

Maxima. --May-July 1950: Daily discharge, 7.1 cfs May 30.

1948 to April 1950: Discharge observed, $365 \mathrm{cfs}$ May 6, 1949; gage height observed, $3.58 \mathrm{ft}$

Feb. 23, 1949 (backwater from ice).

Remarks.--Natural flow affected by irrigation development.

Mean discharge, in cubic feet per second, 1950

\begin{tabular}{|r|r|r|r|r|r|r|r|r|r|r|r|}
\hline Day & \multicolumn{1}{c|}{ May } & \multicolumn{1}{c|}{ June } & \multicolumn{1}{c|}{ July } & Day & \multicolumn{1}{c|}{ May } & \multicolumn{1}{c|}{ June } & \multicolumn{1}{c|}{ July } & Day & May & June & July \\
\hline 1 & 5.6 & 4.8 & 0.6 & 11 & 5.6 & 3.0 & 1.8 & 21 & 5.6 & 1.8 & 1.4 \\
2 & 5.6 & 4.6 & 1.5 & 12 & 5.6 & 3.1 & 1.8 & 22 & 5.2 & .8 & 1.4 \\
3 & 5.6 & 4.6 & 1.4 & 13 & 5.6 & 3.1 & 2.5 & 23 & 5.2 & .7 & 1.5 \\
4 & 5.8 & 4.6 & 1.8 & 14 & 5.0 & 3.1 & 1.7 & 24 & 6.7 & .7 & 2.0 \\
5 & 6.0 & 4.0 & 2.0 & 15 & 5.4 & 2.9 & 1.7 & 25 & 6.7 & .6 & 2.2 \\
6 & 5.6 & 3.4 & 1.6 & 16 & 5.4 & 2.8 & 1.7 & 26 & 6.9 & .5 & 2.6 \\
7 & 5.6 & 3.4 & 1.6 & 17 & 5.4 & 2.5 & 1.6 & 27 & 6.9 & .9 & 1.9 \\
8 & 5.6 & 3.1 & 1.6 & 18 & 5.6 & 2.4 & 1.3 & 28 & 6.9 & 1.1 & 2.5 \\
9 & 5.6 & 3.1 & 1.9 & 19 & 5.4 & 2.2 & 1.2 & 29 & 6.9 & 1.3 & 2.2 \\
10 & 5.6 & 3.0 & 1.8 & 20 & 5.6 & 2.0 & 1.3 & 30 & 7.1 & 1.3 & 1.6 \\
\end{tabular}

Thompson Creek at Riverton, Nebr.

Location. --Lat $40^{\circ} 05^{\prime} 25^{\prime \prime}$, long. $98^{\circ} 45^{\prime} 45^{\prime \prime}$, in NW $\frac{1}{4}$ sec. 2, T. 1 N., R. 13 W. , at bridge on State Highway 3, at west edge of Riverton, half a mile upstream from mouth. Datum of gage is $1,753.38 \mathrm{ft}$ above mean sea level, datum of 1929 . Prior to July 11, 1950, at datum of $1.32 \mathrm{ft}$ higher.

Drainage area. $-295 \mathrm{sq} \mathrm{mi}$.

Gage-height record.--Water-stage recorder graph except for period July 10-31, when once-daily wireweight gage readings were used.

Discharge record. --Stage-discharge relation defined by current-meter measurements below 1,500 efs and extended to peak stage on basis of slope-area measurement.

Maxima. --May-July 1950: Discharge, 12, 200 cfs 3:30 a. m. July 9 (gage height, 13. $22 \mathrm{ft}$, present datum).

1948 to April 1950: Discharge, 3, 260 cfs June 8, 1949 (gage height, 7. 44 ft, present datum).

Miean discharge, in cubic feet per second, 1950

\begin{tabular}{|c|c|c|c|c|c|c|c|c|c|c|c|}
\hline Day & May & June & July & Day & May & June & July & Day & May & June & July \\
\hline $\begin{array}{r}1 \\
2 \\
3 \\
4 \\
5 \\
6 \\
7 \\
8 \\
9 \\
10\end{array}$ & $\begin{array}{l}22 \\
28 \\
29 \\
27 \\
28 \\
26 \\
24 \\
28 \\
24 \\
27\end{array}$ & $\begin{array}{l}26 \\
26 \\
19 \\
20 \\
20 \\
18 \\
19 \\
19 \\
19 \\
20\end{array}$ & $\begin{array}{r}21 \\
23 \\
22 \\
22 \\
20 \\
20 \\
19 \\
20 \\
3,230 \\
132\end{array}$ & $\begin{array}{l}11 \\
12 \\
13 \\
14 \\
15 \\
16 \\
17 \\
18 \\
19 \\
20\end{array}$ & $\begin{array}{l}27 \\
30 \\
29 \\
29 \\
32 \\
30 \\
27 \\
28 \\
28 \\
36\end{array}$ & $\begin{array}{l}20 \\
21 \\
20 \\
20 \\
21 \\
22 \\
23 \\
20 \\
20 \\
23\end{array}$ & $\begin{array}{l}45 \\
39 \\
36 \\
36 \\
42 \\
43 \\
39 \\
39 \\
27 \\
24\end{array}$ & $\begin{array}{l}21 \\
22 \\
23 \\
24 \\
25 \\
26 \\
27 \\
28 \\
29 \\
30 \\
31\end{array}$ & $\begin{array}{l}30 \\
33 \\
32 \\
29 \\
29 \\
32 \\
32 \\
39 \\
44 \\
34 \\
23\end{array}$ & $\begin{array}{l}22 \\
20 \\
22 \\
21 \\
20 \\
21 \\
21 \\
20 \\
20 \\
20 \\
- \\
\end{array}$ & $\begin{array}{l}23 \\
22 \\
26 \\
19 \\
19 \\
22 \\
23 \\
22 \\
23 \\
23 \\
24 \\
\end{array}$ \\
\hline \multicolumn{9}{|c|}{ 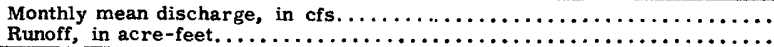 } & $\begin{array}{r}29,5 \\
1,820 \\
\end{array}$ & $\begin{array}{r}20,8 \\
1,240\end{array}$ & $\begin{array}{r}134 \\
8,220 \\
\end{array}$ \\
\hline
\end{tabular}

Gage height, in feet, and discharge, in cubic feet per second, at indicated time, 1950

\begin{tabular}{|c|c|c|c|c|c|c|c|c|c|c|c|}
\hline Hour & $\begin{array}{l}\text { Gage } \\
\text { height }\end{array}$ & Discharge & Hour & $\begin{array}{l}\text { Gage } \\
\text { height }\end{array}$ & Discharge & $\begin{array}{l}\text { Gage } \\
\text { Hour }\end{array}$ & height & Discharge & Hour & $\begin{array}{c}\text { Gage } \\
\text { height }\end{array}$ & Discharge \\
\hline $\begin{array}{c}12 \\
1 \\
2 \\
3 \\
3: 30 \\
4\end{array}$ & $\begin{array}{r}2.08 \\
4.49 \\
7.10 \\
11.30 \\
11.90 \\
9.45\end{array}$ & $\begin{array}{rr} & \\
& 21 \\
& 21 \\
1, & 310 \\
4,550 \\
11,200 \\
12,200 \\
8,600\end{array}$ & $\begin{array}{r}5 \\
6 \\
7 \\
8 \\
9 \\
10\end{array}$ & $\begin{array}{l}\text { July } \\
9.45 \\
8.30 \\
8.13 \\
7.15 \\
5.22 \\
4.30\end{array}$ & $\begin{array}{r}\text { (con.) } \\
8,600 \\
7,320 \\
7,210 \\
6,120 \\
4,020 \\
3,190\end{array}$ & $\begin{array}{c}11 \\
N \\
1 \\
2 \\
3 \\
4 \\
5\end{array}$ & $\begin{array}{l}\quad \text { July } \\
3.45 \\
2.83 \\
2.24 \\
2.05 \\
1.92 \\
1.81 \\
1.75\end{array}$ & $\begin{array}{r}9 \text { (con.) } \\
2,340 \\
1,640 \\
1,090 \\
940 \\
844 \\
767 \\
725\end{array}$ & $\begin{array}{r}6 \\
7 \\
8 \\
9 \\
10 \\
11 \\
12\end{array}$ & $\begin{array}{l}\text { July } \\
1.68 \\
1.61 \\
1.55 \\
1.49 \\
1.41 \\
1.34 \\
1.25\end{array}$ & $\begin{array}{r}9 \text { (con.) } \\
678 \\
636 \\
600 \\
564 \\
516 \\
480 \\
435\end{array}$ \\
\hline
\end{tabular}


Elm Creek at A mboy, Nebr.

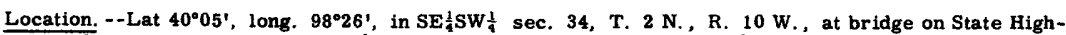
way 3 at east edge on A mboy, $2 \frac{1}{2}$ miles upstream from mouth, and $4 \frac{1}{2}$ miles east of Red Cloud. Datu $n$ of gage is $1,666.18 \mathrm{ft}$ above mean sea level, datum of 1929 .

Drainage area. --54 sq $\mathrm{mi}$.

Gage-height record. --Wire-weight gage read once daily except May 8, July 9, 10, 17, when twice-daily readings were obtained.

Discharge record. --Stage-discharge relation defined by current-meter measurements. Shifting-contro' method used. Discharge for days of no gage-height record interpolated.

Maxima. --May-July 1950: Discharge, $1,380 \mathrm{cfs} 3 \mathrm{p} . \mathrm{m}$. July 9 (gage height, 6. $6 \mathrm{ft}$, from graph based on gage readings).

April 1948 to April 1950: Discharge observed, 994 cfs May 21, 1949 (gage height, $6.02 \mathrm{ft}$ ).

Mean discharge, in cubic feet per second, 1950

\begin{tabular}{|c|c|c|c|c|c|c|c|c|c|c|c|}
\hline Day & May & June & July & Day & May & June & July & Day & May & June & July \\
\hline $\begin{array}{r}1 \\
2 \\
3 \\
4 \\
5 \\
6 \\
7 \\
8 \\
9 \\
10\end{array}$ & $\begin{array}{l}16 \\
16 \\
16 \\
16 \\
21 \\
17 \\
17 \\
17 \\
21 \\
16\end{array}$ & $\begin{array}{l}14 \\
14 \\
14 \\
14 \\
14 \\
14 \\
14 \\
14 \\
14 \\
13\end{array}$ & $\begin{array}{r}14 \\
14 \\
15 \\
18 \\
16 \\
18 \\
15 \\
16 \\
429 \\
66\end{array}$ & $\begin{array}{l}11 \\
12 \\
13 \\
14 \\
15 \\
16 \\
17 \\
18 \\
19 \\
20\end{array}$ & $\begin{array}{l}16 \\
16 \\
17 \\
16 \\
16 \\
16 \\
16 \\
17 \\
19 \\
19\end{array}$ & $\begin{array}{l}14 \\
14 \\
14 \\
14 \\
14 \\
14 \\
14 \\
14 \\
14 \\
14\end{array}$ & $\begin{array}{r}21 \\
19 \\
18 \\
17 \\
16 \\
18 \\
270 \\
23 \\
15 \\
14\end{array}$ & $\begin{array}{l}21 \\
22 \\
23 \\
24 \\
25 \\
26 \\
27 \\
28 \\
29 \\
30 \\
31 \\
\end{array}$ & $\begin{array}{l}18 \\
17 \\
16 \\
16 \\
16 \\
17 \\
17 \\
17 \\
36 \\
26 \\
17\end{array}$ & $\begin{array}{l}14 \\
14 \\
31 \\
14 \\
14 \\
14 \\
14 \\
14 \\
14 \\
14 \\
- \\
\end{array}$ & $\begin{array}{l}13 \\
12 \\
13 \\
14 \\
16 \\
14 \\
14 \\
14 \\
14 \\
14 \\
14\end{array}$ \\
\hline \multicolumn{9}{|c|}{$\begin{array}{l}\text { Monthly mean discharge, in } \text { cfs. } \ldots \ldots \ldots \ldots \ldots \ldots \ldots \ldots \ldots \ldots \ldots \ldots \ldots \ldots \ldots \ldots \ldots \ldots \ldots \ldots \ldots \ldots \ldots \ldots \ldots \ldots \ldots \\
\text { Runoff, in acre-feet. } \ldots \ldots \ldots \ldots \ldots \ldots \ldots \ldots\end{array}$} & $\begin{array}{r}17.9 \\
1,100\end{array}$ & $\begin{array}{r}14.5 \\
865\end{array}$ & $\begin{array}{r}38.7 \\
2,386\end{array}$ \\
\hline
\end{tabular}

Gage height, in feet, and discharge, in cubic feet per second, at indicated time, 1950

\begin{tabular}{|c|c|c|c|c|c|c|c|c|c|c|c|}
\hline Hour & $\begin{array}{c}\text { Gage } \\
\text { height }\end{array}$ & Discharge & Hour & $\begin{array}{c}\text { Gage } \\
\text { height }\end{array}$ & Discharge & Hour & $\begin{array}{c}\text { Gage } \\
\text { height }\end{array}$ & Discharge & Hour & $\begin{array}{c}\text { Gage } \\
\text { height }\end{array}$ & Discharge \\
\hline & \multicolumn{2}{|c|}{ July 9} & \multirow{3}{*}{$\begin{array}{l}10 \\
N\end{array}$} & \multicolumn{2}{|c|}{ July 9 (con } & & \multicolumn{2}{|c|}{ July 9 (con. } & & \multicolumn{2}{|c|}{ July 10} \\
\hline $1 \mathrm{a}$ & 0.93 & 16 & & 3.75 & 286 & 4 & 6.37 & 1,190 & 3 & 2.05 & 70 \\
\hline 4 & 1.07 & 20 & & 6.16 & 1,060 & 6 & 5.10 & 640 & 6 & 1.69 & 50 \\
\hline 6 & 1.37 & 32 & 2. & 6.57 & 1,360 & 8 & 4.13 & 372 & 9 & 1.53 & 42 \\
\hline 8 & 2.10 & 69 & 3 & 6.60 & 1,380 & 10 & 3.37 & 221 & $\mathrm{~N}$ & 1.39 & 36 \\
\hline & & & & & & 12 & 2.69 & 127 & 6 & 1.23 & 29 \\
\hline & & & & & & & & & 12 & 1.12 & 25 \\
\hline
\end{tabular}


Big Blue River near Crete, Nebr.

pcation. --Lat $40^{\circ} 35^{\prime} 40^{\prime \prime}$, long. $96^{\circ} 57^{\prime} 35^{\prime \prime}$, in $S \frac{1}{2}$ sec. 3, T. 7 N., R. 4 E., at bridge on State Highway

82, 1.8 miles south from Missouri Pacific Railroad station in Crete, 3.3 miles downstream from Walnut Creek, 3. 6 miles upstream from Squaw Creek. Datum of gage is $1,311.5 \mathrm{ft}$ above mean sea level. datum of 1929 .

Orainage area. $--2,680 \mathrm{sq} \mathrm{mi}$.

jage-height record. --Wire-weight gage read twice daily. Gage heights above 12 ft obtained from graph based on gage readings.

Discharge record. --Stage-discharge relation defined by current-meter measurements. Stage-discharge relation below about $12 \mathrm{ft}$ affected by backwater from power dam downstream; discharge computed only for stages above $12 \mathrm{ft}$.

Maxima.--May-July 1950: Discharge, 27,600 cfs 1 p. m. July 10 (gage height, 28. 74 ft).

$194 \overline{5}$ to April 1950: Discharge, $20,900 \mathrm{cfs}$ Mar. 8, 1949 (gage height, 27. $0 \mathrm{ft}$ ).

Mean discharge, in cubic feet per second, 1950

\begin{tabular}{|c|c|c|c|c|c|c|c|c|c|c|c|}
\hline Day & May & June & July & Day & May & June & July & Day & May & June & July \\
\hline $\begin{array}{r}\mathbf{1} \\
\mathbf{2} \\
\mathbf{3} \\
\mathbf{4} \\
\mathbf{5} \\
\mathbf{6} \\
\mathbf{7} \\
\mathbf{8} \\
\mathbf{9} \\
\mathbf{1 0}\end{array}$ & 1,790 & $\begin{array}{l}2,630 \\
1,670\end{array}$ & 15,900 & $\begin{array}{l}11 \\
12 \\
13 \\
14 \\
15 \\
16 \\
17 \\
18 \\
19 \\
20\end{array}$ & $\begin{array}{r}3,740 \\
4,670 \\
4,190 \\
2,030 \\
968\end{array}$ & & $\begin{array}{r}10,800 \\
6,420 \\
4,660 \\
4,150 \\
3,810 \\
4,090 \\
4,870 \\
2,860 \\
1,460\end{array}$ & $\begin{array}{l}21 \\
22 \\
23 \\
24 \\
25 \\
26 \\
27 \\
28 \\
29 \\
30 \\
31\end{array}$ & $\begin{array}{l}1,470 \\
3,580\end{array}$ & & \\
\hline
\end{tabular}

Gage height, in feet, and discharge, in cubic feet per second, at indicated time, 1950

\begin{tabular}{|c|c|c|c|c|c|c|c|c|c|c|c|}
\hline Hour & $\begin{array}{c}\text { Gage } \\
\text { height }\end{array}$ & Discharge & Hour & $\begin{array}{c}\begin{array}{c}\text { Gage } \\
\text { height }\end{array} \\
\end{array}$ & Discharge & Hour & $\begin{array}{c}\text { Gage } \\
\text { height }\end{array}$ & Discharge & Hour & $\begin{array}{c}\text { Gage } \\
\text { height }\end{array}$ & Discharge \\
\hline $\begin{array}{r}2 \\
4 \\
6 \\
8 \\
10\end{array}$ & $\begin{array}{l}12.00 \\
14.40 \\
19.20 \\
26.80 \\
28.20\end{array}$ & $\begin{array}{r}\text { uly } 10 \\
1,440 \\
2,400 \\
4,570 \\
19,200\end{array}$ & $\begin{array}{r}2 \\
4 \\
6 \\
8\end{array}$ & $\begin{array}{l}\text { July } \\
28.70 \\
28.55 \\
27.84 \\
27.15 \\
26.60\end{array}$ & \begin{tabular}{|r|} 
(con.) \\
24,400 \\
20,700 \\
19,800 \\
19,300 \\
18
\end{tabular} & $\begin{array}{r}N \\
4 \\
8 \\
12\end{array}$ & $\begin{array}{l}\text { July } 1 \\
23.80 \\
23.15 \\
22.60 \\
22.15\end{array}$ & $\begin{array}{r}(\operatorname{con} .) \\
10,200 \\
9,040 \\
8,210 \\
7,600\end{array}$ & $\begin{array}{r}6 \\
N \\
6 \\
12\end{array}$ & $\begin{array}{l}\text { Ju } \\
19.65 \\
19.23 \\
18.92 \\
18.76\end{array}$ & $\begin{array}{r}\text { y } 13,890 \\
4,590 \\
4,410 \\
4,330\end{array}$ \\
\hline $\begin{array}{r}10 \\
\mathbf{N} \\
1\end{array}$ & $\begin{array}{l}28.20 \\
28.68 \\
28.74\end{array}$ & $\begin{array}{l}25,100 \\
27,300 \\
27,600\end{array}$ & $\begin{array}{r}10 \\
12 \\
\\
4 \\
8\end{array}$ & $\begin{array}{r}26.60 \\
26.10 \\
25.25 \\
24.50 \\
\end{array}$ & $\begin{array}{r}18,400 \\
16,600 \\
11 \\
13,800 \\
11,700\end{array}$ & $\begin{array}{r}6 \\
N \\
6 \\
12 \\
\end{array}$ & $\begin{array}{l}21.75 \\
21.22 \\
20.59 \\
20.04\end{array}$ & $\begin{array}{r}12 \\
7,900 \\
6,460 \\
5,790 \\
5,240\end{array}$ & $\begin{array}{r}N \\
12\end{array}$ & $\begin{array}{r}\text { Jul } \\
18.43 \\
17.88\end{array}$ & $\begin{array}{r}4,160 \\
3,890\end{array}$ \\
\hline
\end{tabular}


Big Blue River at Barneston, Nebr.

Location. --Lat $40^{\circ} 03^{\prime}$, long. $9^{\circ} 35^{\prime}$, in $\mathrm{NE} \frac{1}{4} \mathrm{SW} \frac{1}{4}$ sec. 13, T. $1 \mathrm{~N}$., R. $7 \mathrm{E}$., in tailwater of power plant, three-quarters of a mile northwest of Barneston, 2 miles upstream from Plum Creek, and 5 miles upstream from Nebraska-Kansas State line.

Drainage area. $--4,420 \mathrm{sq} \mathrm{mi}$.

Gage-height record. --Water-stage recorder graph.

Discharge record. --Stage-discharge relation defined by current-meter measurements. Shifting-control method used.

Maxima. --May-July 1950: Discharge, 21, 300 cfs 4 p. m. May 10 (gage height, $25,67 \mathrm{ft}$ ).

1932 to April 1950: Discharge, 57, 700 cfs June 9, 1941 (gage height, $34.3 \mathrm{ft}$ ).

Remarks. --Low flow regulated by power plant at gage, which has pondage of about 1, 500 acre-ft. High flow occasionally affected for short periods by operation of trash gates.

Mean discharge, in cubic feet per second, 1950

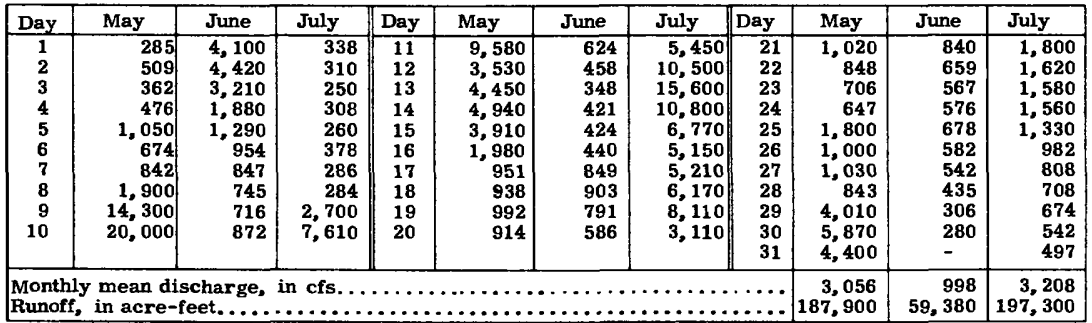

Gage height, in feet, and discharge, in cubic feet per second, at indicated time, 1950

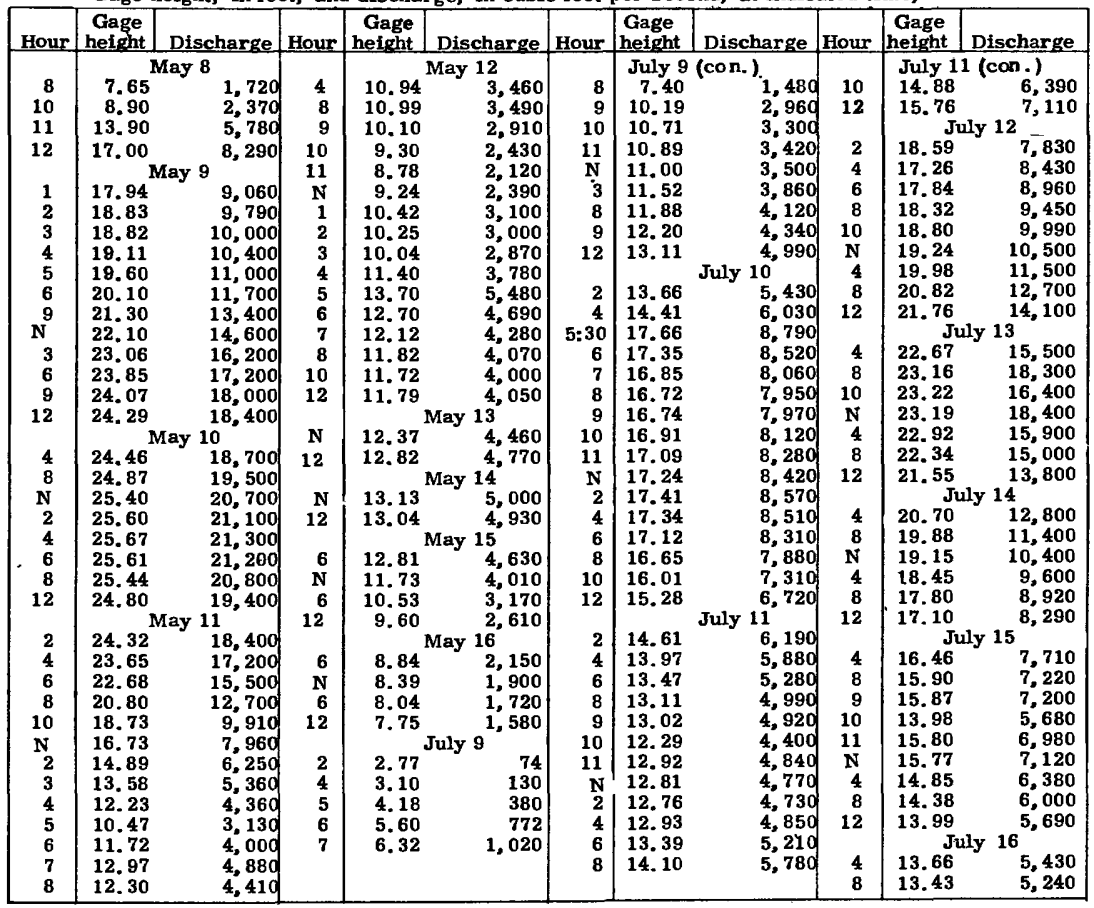


Little Blue River near Endicott, Nebr.

Location. =-Lat $40^{\circ} 05^{\prime} 10^{\prime \prime}$, long. $97^{\circ} 08^{\prime} 10^{\prime \prime}$, in sec. 6, T. 1 N., R. 3 E., 300 ft downstream from county highway bridge, $1 \frac{1}{2}$ miles upstream from Chicago, Burlington \& Quincy Railroad bridge, and 2 miles northwest of Endicott.

Drainage area. $--2,340 \mathrm{sq} \mathbf{m i}$.

Gage-height record. --Water-stage recorder graph. N, gage-height record May 1.

Discharge record. --Stage-discharge relation defined by current-meter measurements. Shifting-control method used.

Maxima. --May-July 1950: Discharge, 12,100 cfs 9 a. m. July 9 (gage height, $14.08 \mathrm{ft}$ ).

1908-15, 1929 to April 1950: Discharge, 31, 000 cfs June 9, 1941 (gage height, 16. 23 ft), from rating curve extended above $20,000 \mathrm{cfs}$.

Mean discharge, in cubic feet per second, 1950

\begin{tabular}{|c|c|c|c|c|c|c|c|c|c|c|c|}
\hline Day & May & June & July & Day & May & June & July & Day & May & June & Iuly \\
\hline $\begin{array}{r}1 \\
2 \\
3 \\
4 \\
5 \\
6 \\
7 \\
8 \\
9 \\
10\end{array}$ & $\begin{array}{r}160 \\
158 \\
156 \\
154 \\
273 \\
656 \\
339 \\
300 \\
4,050 \\
1,190\end{array}$ & $\begin{array}{r}1,300 \\
1,760 \\
970 \\
598 \\
477 \\
397 \\
352 \\
317 \\
295 \\
275\end{array}$ & \begin{tabular}{r|}
234 \\
210 \\
187 \\
170 \\
164 \\
162 \\
172 \\
168 \\
7,420 \\
4,050
\end{tabular} & $\begin{array}{l}11 \\
12 \\
13 \\
14 \\
15 \\
16 \\
17 \\
18 \\
19 \\
20\end{array}$ & $\begin{array}{l}604 \\
810 \\
458 \\
329 \\
282 \\
254 \\
232 \\
219 \\
302 \\
232\end{array}$ & $\begin{array}{r}\mathbf{2 6 1} \\
250 \\
239 \\
230 \\
219 \\
214 \\
510 \\
1,770 \\
\mathbf{5 1 4} \\
\mathbf{3 5 9}\end{array}$ & $\begin{array}{r}3,650 \\
9,490 \\
6,890 \\
2,820 \\
1,050 \\
774 \\
4,430 \\
8,300 \\
5,030 \\
1,850\end{array}$ & $\begin{array}{l}21 \\
22 \\
23 \\
24 \\
25 \\
26 \\
27 \\
28 \\
29 \\
30 \\
31\end{array}$ & $\begin{array}{r}230 \\
212 \\
228 \\
193 \\
805 \\
445 \\
329 \\
250 \\
2,670 \\
6,250 \\
1,230\end{array}$ & $\begin{array}{l}282 \\
245 \\
252 \\
232 \\
364 \\
282 \\
225 \\
219 \\
208 \\
185 \\
-\end{array}$ & $\begin{array}{r}1,000 \\
792 \\
656 \\
572 \\
536 \\
746 \\
722 \\
548 \\
470 \\
430 \\
402\end{array}$ \\
\hline \multicolumn{9}{|c|}{ 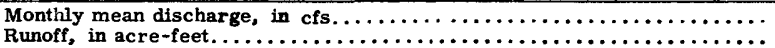 } & $\begin{array}{r}774 \\
47,600 \\
\end{array}$ & $\begin{array}{r}460 \\
27,370 \\
\end{array}$ & $\begin{array}{r}2,068 \\
127,100 \\
\end{array}$ \\
\hline
\end{tabular}

Gage height, in feet, and discharge, in cubic feet per second, at indicated time, 1950

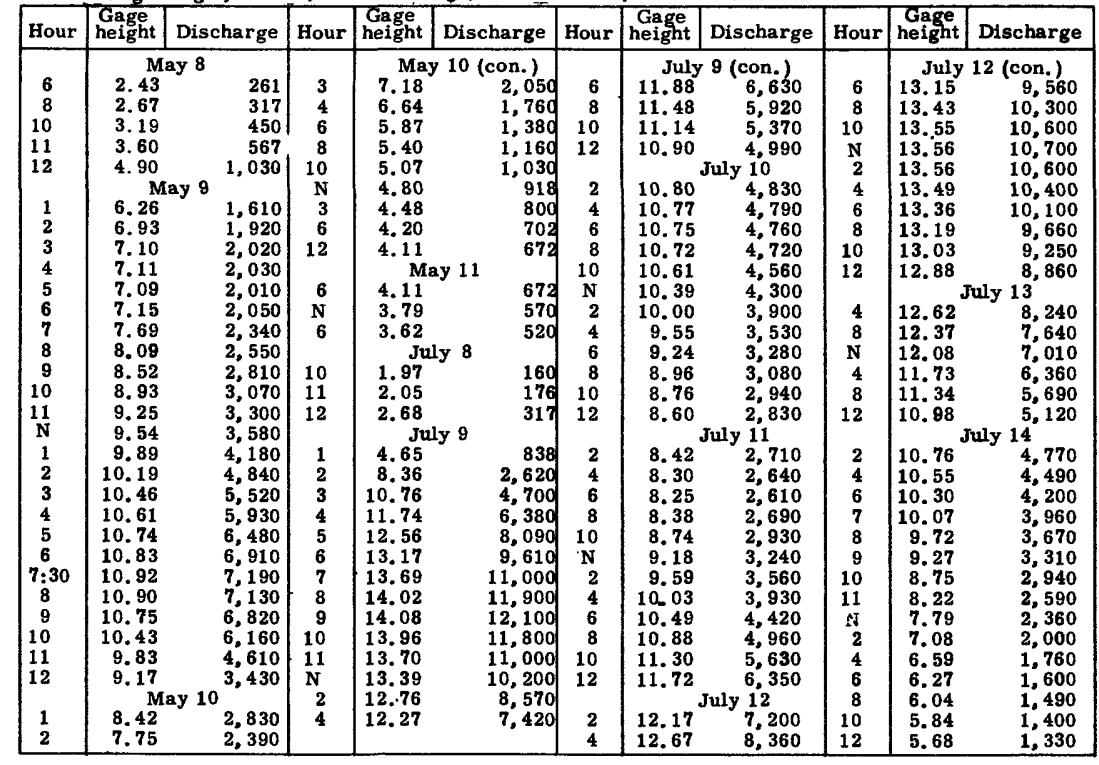




\section{SUMMARY OF FLOOD STAGES AND DISCHARGES}

The results of the determinations of maximumflood flows a $t$ existing stream-gaging stations and other places on streams in the area covered by this report are summarized and presented in table 3, "Summary of flood stages and discharges." The reference number in this table is applicable to figure 50and will aid in identifying the place where the discharge was determined.

The maximum discharges at existing gaging stations were obtained as a part of the station record, from a stage-discharge relation. The maximum discharge at miscellaneous points was obtained by special methods, such as those noted under "Measure ment of flood discharge." These are indicated by headnote in table 3. Gaging stations and miscellaneous points can be identi fied in table 3 by the entry in the column "Period of record": a period of record is shown for all gaging stations, whereas a dash indicates that the point is miscellaneous, that is, no record of discharge has been collected systematically at that point.

The maximum flood previously known may include a flood antedating the period of $\mathrm{record}$, the general aim being to list the highest flood for which the discharge is known. Information relating to previous maxima is contained in the footnotes.

Figure 61 is a graph showing relation of unit discharge to size of drainage area. The figure provides a convenient method for comparing flood discharges from drainage areas that differ widel: in size; it does not bring out the comparative influence of topographical or other basin characteristics that may influence floot discharge. 


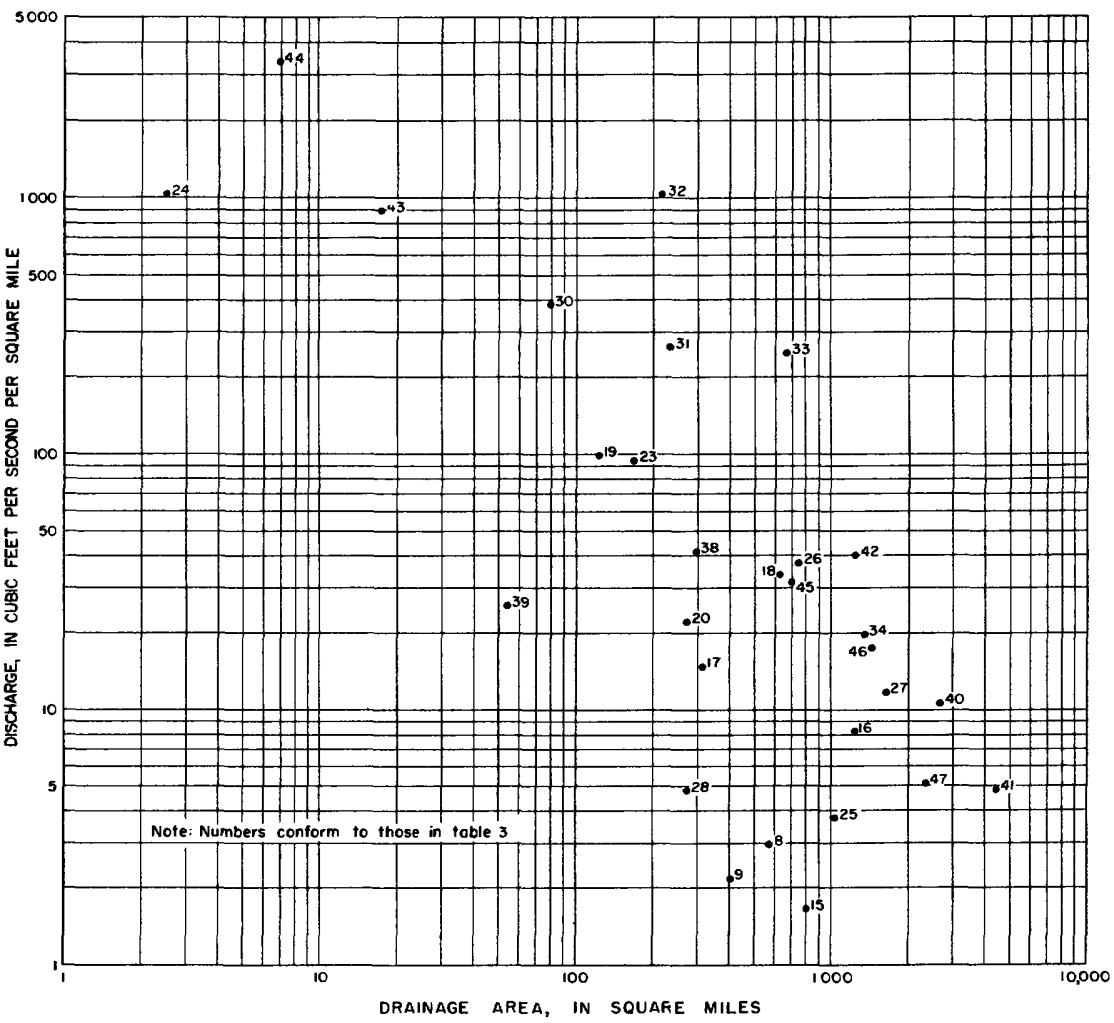

FrgURe 61.-Relation of unit discharge to size of drainage area. 
Table 3. --Summary of flood stages and discharges

[Maximum discharges for the floods of May-July 1950 were obtained from gaging-station records, except as otherwise indicated by the following symbols: S, slope area measurements; $C$, contracted-opening measurement; $D$, computed flow over highway embankment; $\%$, computed flow through culvert]

\begin{tabular}{|c|c|c|c|c|c|c|c|c|c|c|c|}
\hline \multirow{2}{*}{$\begin{array}{l}\text { No. } \\
\text { on } \\
\text { fig. } 1\end{array}$} & \multirow[b]{2}{*}{ Stream and place of determination } & \multirow{2}{*}{$\begin{array}{c}\text { Drainage } \\
\text { area } \\
\text { (square } \\
\text { miles) }\end{array}$} & \multirow{2}{*}{$\begin{array}{l}\text { Period } \\
\text { of } \\
\text { record }\end{array}$} & \multicolumn{4}{|c|}{ Maximum flood previously known } & \multicolumn{4}{|c|}{ Maximum during May-July 1950} \\
\hline & & & & Date & \begin{tabular}{|c|}
$\begin{array}{c}\text { Gage } \\
\text { height } \\
\text { (feet) }\end{array}$ \\
\end{tabular} & $\begin{array}{c}\text { Discharge } \\
\text { (cfs) }\end{array}$ & $\begin{array}{l}\text { Cfs per } \\
\text { square } \\
\text { mile }\end{array}$ & Date and hour & \begin{tabular}{|c|}
$\begin{array}{l}\text { Gage } \\
\text { height } \\
\text { (feet) }\end{array}$ \\
\end{tabular} & $\begin{array}{c}\text { Discharge } \\
\text { (cfs) }\end{array}$ & $\begin{array}{l}\text { Cfs per } \\
\text { square } \\
\text { mile }\end{array}$ \\
\hline & MISSOURI RIVER MAIN STEM & & & & & & & & & & \\
\hline $\begin{array}{l}1 \\
2 \\
3\end{array}$ & $\begin{array}{l}\text { Missouri River at Omaha, Nebr. } \\
\text { Missouri River at Nebraska City, Nebr. } \\
\text { Missouri River at Rulo, Nebr. } \\
\text { PLATTE RIVER BASIN }\end{array}$ & $\begin{array}{l}322,800 \\
414,400 \\
418,905\end{array}$ & $\begin{array}{l}1928- \\
1929- \\
1949-\end{array}$ & $\mid$\begin{tabular}{cc|} 
Apr. 12,1943 \\
June 14,1944 \\
Apr. 29,1950
\end{tabular} & $\begin{array}{c}222.45 \\
19.70 \\
21.6\end{array}$ & $\begin{array}{l}200,000 \\
214,000 \\
185,000\end{array}$ & : & $\begin{array}{l}\text { June } 20,3 \mathrm{a} . \mathrm{m} . \\
\text { May } 9,4: 30 \mathrm{a} . \mathrm{m} \\
\text { May } 10,6 \mathrm{a} . \mathrm{m} .\end{array}$ & $\begin{array}{l}14.00 \\
18.60 \\
21.55\end{array}$ & $\begin{array}{r}96,900 \\
147,000 \\
172,000\end{array}$ & $\begin{array}{l}- \\
-\end{array}$ \\
\hline $\begin{array}{l}4 \\
5\end{array}$ & $\begin{array}{l}\text { Platte River near Grand Island, Nebr. } \\
\text { Platte River near Duncan, Nebr. }\end{array}$ & $\begin{array}{l}59,500 \\
61,600\end{array}$ & $\begin{array}{l}1933- \\
1895-1915 \\
1928-\end{array}$ & $\begin{array}{l}\text { June } 6,1935 \\
\text { June } 23,1905\end{array}$ & 5.99 & $\begin{array}{r}30,000 \\
\text { e51, } 100\end{array}$ & - & $\begin{array}{l}\text { May } 9,3: 30 \text { a. } \mathrm{m} \\
\text { May } 11,1: 15 \mathrm{p} . \mathrm{m}\end{array}$ & $\begin{array}{l}3.60 \\
3.10\end{array}$ & $\begin{array}{l}4,310 \\
4,150\end{array}$ & $\dot{-}$ \\
\hline $\begin{array}{l}6 \\
7\end{array}$ & $\begin{array}{l}\text { Platte River at North Bend, Nebr. } \\
\text { Platte River near Ashland, Nebr. }\end{array}$ & $\overline{83}, 800$ & $\begin{array}{l}1949- \\
1928-\end{array}$ & $\begin{array}{l}\text { June } 24,1947 \\
\text { June } 12,1944\end{array}$ & - & $\begin{array}{r}f 90,000 \\
g 107,000\end{array}$ & - & $\begin{array}{l}\text { July } 12,5 \text { p. m. } \\
\text { July } 20,2: 30 \text { a. } \mathrm{m}\end{array}$ & $\begin{array}{l}5.3 \\
6.30\end{array}$ & $\begin{array}{l}25,000 \\
47,900\end{array}$ & - \\
\hline 8 & Wood River near Gibbon, Nebr. & 572 & $1949-$ & June 9,1949 & 15. 63 & 1,600 & 2. 80 & July $10,3-5$ p. m. & 15. 74 & 1,680 & 2.94 \\
\hline $\begin{array}{r}9 \\
10\end{array}$ & $\begin{array}{l}\text { Prairie Creek near Silver Creek, Nebr. } \\
\text { Middle Loup River at St. Paul, Nebr. }\end{array}$ & $\begin{array}{r}406 \\
7,720\end{array}$ & $\begin{array}{l}1949- \\
1895-97,1899 \\
1903,1928-\end{array}$ & $\begin{array}{l}\text { Sept. } 12,1949 \\
\text { June } 23,1947\end{array}$ & $\begin{array}{r}3.03 \\
10.69\end{array}$ & $\begin{array}{r}127 \\
72,000\end{array}$ & .31 & $\begin{array}{l}\text { July } 13, \text { noon } \\
\text { July } 9,11: 30 \text { a. } m\end{array}$ & $\begin{array}{l}6.62 \\
6.51\end{array}$ & $\begin{array}{r}889 \\
9,600\end{array}$ & 2. 19 \\
\hline 11 & Loup River near Genoa, Nebr & 14,440 & 1928 & June 2 & 10 & & - & July 9 , noon & 7.92 & 39,300 & - \\
\hline 12 & Loup River & & $\begin{array}{c}1894-1915 \\
1931,1933-\end{array}$ & June 2 & hi & & - & July 10,2 & $\mathrm{hg} 0$ & 100 & - \\
\hline 13 & North Loup River near St. Paul, Nebr. & 4,460 & $\begin{array}{r}1895-97,1899 \\
1903,1928-\end{array}$ & June 6,1896 & - & 90,000 & - & July $9,5: 30$ a. m. & 4.37 & 16,800 & - \\
\hline $\begin{array}{l}14 \\
15\end{array}$ & $\begin{array}{l}\text { Spring Creek at Cushing, Nebr. } \\
\text { Cedar River near Spalding, Nebr. }\end{array}$ & -794 & $\begin{array}{l}1949- \\
1945-\end{array}$ & $\begin{array}{l}\text { June } 27,1949 \\
\text { June } 23,1947\end{array}$ & $\begin{array}{l}5.79 \\
7.50\end{array}$ & $\begin{array}{r}448 \\
4.000\end{array}$ & 5.04 & $\begin{array}{l}\text { July } 22,11: 30 \mathrm{p} . \mathrm{m} \\
\text { July } 8,9: 30 \mathrm{p} . \mathrm{m}\end{array}$ & $\begin{array}{r}15.00 \\
5.12\end{array}$ & $\begin{array}{l}2,560 \\
1,320\end{array}$ & 1.66 \\
\hline 16 & on, Nebr. & 1,220 & $1931-32,1940-$ & Feb. 28,1948 & 9.40 & 9,760 & 8.00 & $9,1: 3 c$ & 9.64 & 10,100 & 8.28 \\
\hline 17 & Beaver Creek at Loret to, Nebr. & $\begin{array}{l}311 \\
627\end{array}$ & $1944-$ & Feb. 28, 1948 & 9.81 & 1807 & 2.59 & June 2,11 a. $\mathrm{m}$ & 11.74 & 4,570 & $\begin{array}{l}14.7 \\
33.8\end{array}$ \\
\hline $\begin{array}{l}18 \\
19\end{array}$ & $\begin{array}{l}\text { Beaver Creek at Genoa, Nebr. } \\
\text { Shell Creek at Newman Grove, Nebr. }\end{array}$ & $\begin{array}{l}627 \\
122\end{array}$ & $\begin{array}{l}1940- \\
1949-\end{array}$ & June 21, 1942 & 15.5 & 3,930 & 6.27 & $\begin{array}{l}\text { July } 19,2: 30 \mathrm{a} . \mathrm{m} \\
\text { July } 18,1 \mathrm{p} . \mathrm{m}\end{array}$ & $\begin{array}{r}18.70 \\
\text { j20.20 }\end{array}$ & $\begin{array}{l}21,200 \\
12,000\end{array}$ & $\begin{array}{l}33.8 \\
98.4\end{array}$ \\
\hline 20 & Shell Creek near Columbus, Nebr. & 270 & $1947-$ & June 2,1947 & 21.7 & 4,600 & 17.0 & June $3,12: 30$ p. $\mathrm{m}$ & 21.38 & 5,970 & 22.1 \\
\hline $\begin{array}{l}21 \\
22\end{array}$ & $\begin{array}{l}\text { Elkhorn River at Norfolk, Nebr. } \\
\text { Elkhorn River at Waterloo, Nebr. }\end{array}$ & $\begin{array}{l}2,790 \\
6,900\end{array}$ & $\left|\begin{array}{l}1945- \\
1911-13,1928-\end{array}\right|$ & $\left|\begin{array}{l}\text { June } 26,1947 \\
\text { June } 12,1944\end{array}\right|$ & $\begin{array}{l}\mathrm{k} 11.1 \\
\mathrm{~m} 16.6\end{array}$ & $\begin{array}{r}12,600 \\
100,000\end{array}$ & - & $\begin{array}{l}\text { July } 19,9 \text { a. } \mathrm{m} \\
\text { July } 20,5 \text { a. } \mathrm{m} .\end{array}$ & $\begin{array}{l}\text { e8. } 35 \\
\text { n7.08 }\end{array}$ & $\begin{array}{r}7,080 \\
13,400\end{array}$ & - \\
\hline
\end{tabular}




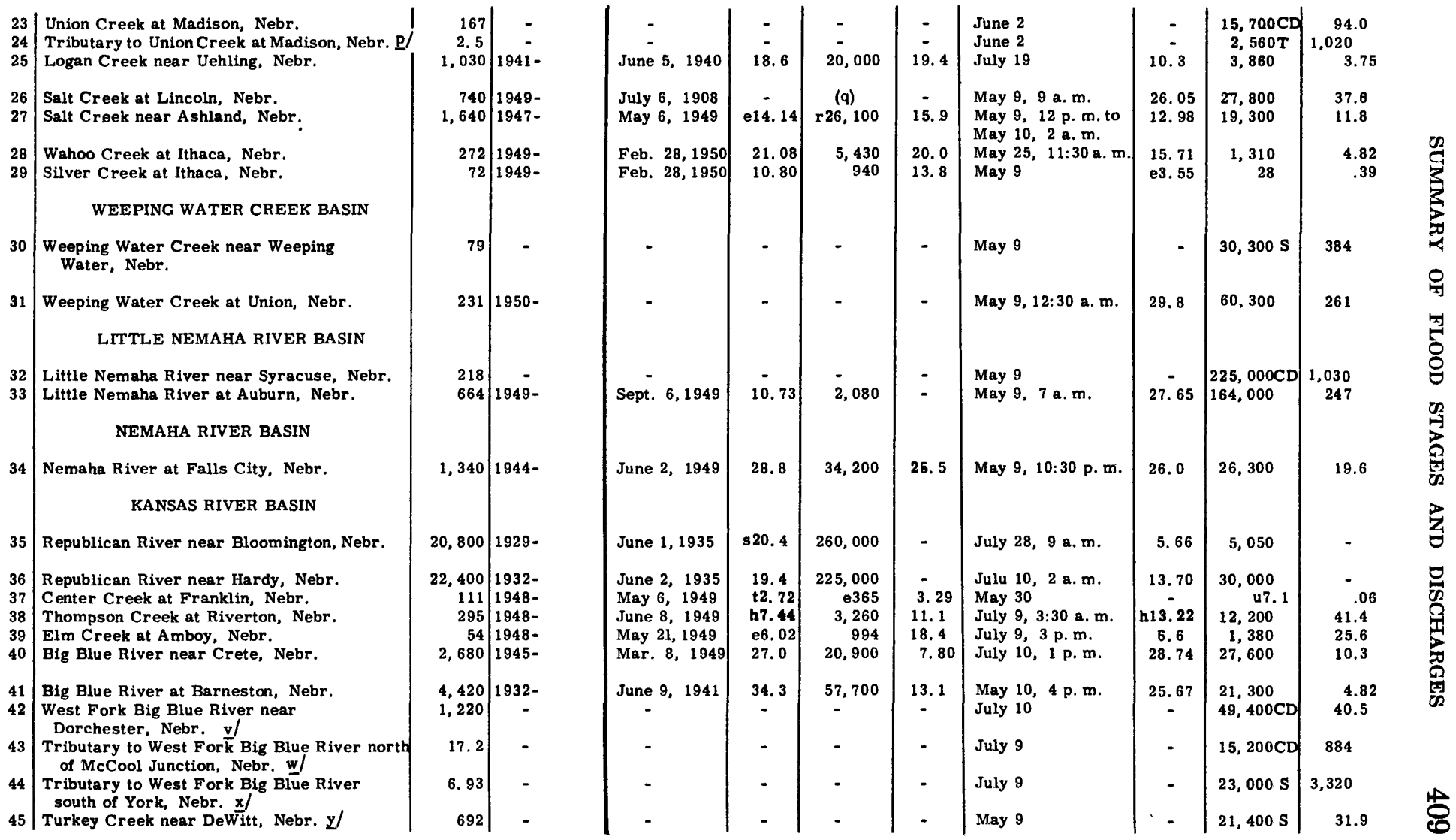


Table 3,--Summary of flood stages and discharges--Continued.

\begin{tabular}{|c|c|c|c|c|c|c|c|c|c|c|c|}
\hline \multirow{2}{*}{$\begin{array}{l}\text { No. } \\
\text { on } \\
\text { fig. } 1\end{array}$} & \multirow[b]{2}{*}{ Stream and place of determination } & \multirow{2}{*}{$\begin{array}{c}\text { Drainage } \\
\text { area } \\
\text { (square } \\
\text { mules) }\end{array}$} & \multirow{2}{*}{$\begin{array}{l}\text { Period } \\
\text { of } \\
\text { record }\end{array}$} & \multicolumn{4}{|c|}{ Maximum flood previously known } & \multicolumn{4}{|c|}{ Maximum during May-July 1950} \\
\hline & & & & Date & $\begin{array}{l}\begin{array}{l}\text { Gage } \\
\text { height } \\
\text { (feet) }\end{array} \\
\end{array}$ & $\begin{array}{c}\text { Discharge } \\
\text { (cfs) }\end{array}$ & \begin{tabular}{|c|}
$\begin{array}{c}\text { Cfs per } \\
\text { square } \\
\text { mile }\end{array}$ \\
\end{tabular} & Date and hour & $\begin{array}{c}\text { Gage } \\
\text { height } \\
\text { (feet) }\end{array}$ & \begin{tabular}{|c} 
Discharge \\
(cfs)
\end{tabular} & $\begin{array}{l}\text { Cfs per } \\
\text { square } \\
\text { mile } \\
\end{array}$ \\
\hline $\begin{array}{l}46 \\
47\end{array}$ & $\begin{array}{l}\text { KANSAS RIVER BASIN--Continued } \\
\text { Little Blue River at Hebron, Nebr. z/ } \\
\text { Little Blue River near Endicott, Nebr. }\end{array}$ & $\begin{array}{l}1,440 \\
2,340\end{array}$ & $\frac{-}{1908-15,1929-}$ & June 9,1941 & 16.23 & $\overline{31,000}$ & $\overline{13 .} 2$ & $\begin{array}{l}\text { About July } 11 \\
\text { July } 9,9 \text { a. } \mathrm{m} \text {. }\end{array}$ & 14.08 & $\begin{array}{l}24,800 \mathrm{C} \\
12,100\end{array}$ & $\begin{array}{l}17.2 \\
5.17\end{array}$ \\
\hline
\end{tabular}

a Occurred Apr. 13, 1943. Maximum stage known, 24.65 ft.Apr. 25, 1881, ice jam b Maximum gage height, $25.8 \mathrm{ft}$ on Mar. 6, 1949 (ice jam).

c Occurred Apr. 30,1950 .

p Determination made at culvert on U.S. Highway 81 in NW sec. 8, T. 21 N., R. 1 W. q Flood of May 9,1950, greatest since that of July 6,1908, which may have been equal or greater.

d Maximum gage height, 6.03 ft on Mar. 4, 1949 (backwater from ice).

e Observed.

f Greater than flood of June 1886.

g Greatest known flood since 1883 .

h Present datum.

i May have been exceeded by flood in May 1945, for which no record was obtained.

j Flood of June 2, 1950, reached stage of $20.13 \mathrm{ft}$ (discharge, 11, $600 \mathrm{cfs}$ ).

k Maximum gage height, $12.25 \mathrm{ft}$ on Feb. 28, 1949 (backwater from ice).

m Greatest flood known.

n Occurred at 11 a. $m$. July 22

$r$ May include some overflow from Platte River.

$s$ Site then in use.

t Maximum gage height observed, $3.58 \mathrm{ft}$ on Feb. 23, 1949 (backwater from ice).

u Mean daily discharge.

$\checkmark$ At bridge on U. S. Highway 6 .

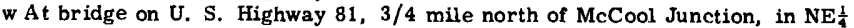
sec. 13, T. 9 N., R. 3 W.

$x$ Near center of sec, $6, T, 9 \mathrm{~N}$, , R, $2 \mathrm{~W}$, about 6 miles south of York.

y In NW $\frac{1}{4}$ sec. 15, T. 5 N., R. 4 E., downstream from Swan Creek.

2 At bridge on U. S. Highway 81 . 
Page

351

A mboy, Nebr. , Elm Creek at ........ 402 Ashland, Nebr., Platte River near 377 Salt Creek near ................ 395 Auburn, Nebr., inundation of highway

hear .............. 359,fig. 55

Little Nemaha River at . . . . . . . . 398

Barneston, Nebr., Big Blue River at .. . 404 Beaver Creek at Genoa, Nebr. . . . . . . 389 at Loretto, Nebr. . . . . . . . . . . . 388 Beaver Crossing, Nebr., flood at 363,fig. 59 3ig Blue River at Barneston, Nebr. . . . 404 near Crete, Nebr. ............403 Bloomington, Nebr., Republican River

near ................. 399 Cedar River near Fullerton, Nebr. . . . 386 near Spalding, Nebr. . . . . . . . 385 Center Creek at Franklin, Nebr. ..... 401 Columbus, Nebr., Loup River at ..... 382 Shell Creek near . . . . . . . . . 391 Crete, Nebr., Big Blue River near . . . 403 Cushing, Nebr., Spring Creek at . . . . 384 Damages, flood ..............364-366 Duncan, Nebr., Platte River near. .... 375 ikhorn River at Norfolk, Nebr. . . . . 392 at Waterloo, Nebr. . . . . . . . . . 393 Im Creek at Amboy, Nebr. . . . . . . . 402 Endicott, Nebr. , Little Blue River near 405 Explanation of data .............. 371 Palls City, Nebr., Nemaha River at . . 399 lood damages.............. 364-366 lood discharges, measurement of . .370-321 lood stages and discharges, summary

of ................406-407

loods, general description of . . . . 352-363

ranklin, Nebr., Center Creek at .... 401 illerton, Nebr., Cedar River near.... 386 ieneral description of the floods . . . 352-363 ienoa, Nebr., Beaver Creek at ...... 389 damage to highway near ......363, fig. 60 Loup River near. . . . . . . . . . ... 381 Loup River power canal near ........ 387 tibbon, Nebr., Wood River near ...... 378 rrand Island, Nebr. , Platte River near. . 374 lardy, Nebr., Republican River near... 400 itroduction. . . . . . . . . . . . 351-352 sohyetal map of Shell Creek basin 359,fig. 56 southeastern Nebraska and north-

eastern Kansas......... 361,fig. 57 southeastern Nebraska and western

Iowa ............. 355,fig. 52 thaca, Nebr. , Silver Creek at . . . . ... 396 Wahoo Creek at .............. 396 incoln, Nebr. , Salt Creek at . . . . . . 394 ittle Blue River near Endicott, Nebr. . 405 ittle Nemaha River at Auburn, Nebr... . 398 hydrograph of . . . . . . 354 ,fig. 51 ogan Creek near Uehling, Nebr....... 394 oretto, Nebr., Beaver Creek at ...... 388
Loup River at Columbus, Nebr. . . . 382 hydrograph of ........ 354,fig. 51 near Genoa, Nebr........... 381 power canal near Genoa, Nebr. . . 387 Measurement of flood discharges 370-371 Meteorologic conditions .......366-370 Middle Loup River at St. Paul, Nebr. . 380 Missouri River at Nebraska City,

Nebr. ................ 373

at Omaha, Nebr. .......... 373

at Rulo, Nebr. . ............ 374

Nebraska City, Nebr. , Missouri

River at ............ 373

Nemaha River at Falls City, Nebr. . . 399

Newman Grove, Nebr., Shell Creek at . . . . . . . . . . . . 390

Norfolk, Nebr., Elkhorn River at ... 392

North Bend, Nebr., Platte River at .. 376 North Loup River near St. Paul, Nebr. 383 Omaha, Nebr., Missour River at... 373 Platte River at North Bend, Nebr.... 376

near Ashland, Nebr. . . . . . . . 377

near Duncan, Nebr. .......... 375

near Grand Island, Nebr. . . . . . . 374

Prairie Creek near Silver Creek, Nebr. . . . . . . . . . . . . . 379

Republican River near Bloomington,

Nebr. . . . . . . . . . . . . 399

near Hardy, Nebr...........400

Riverton, Nebr., Thomson Creek at. 401 Rulo, Nebr., Missouri River at . . . . 374 St. Paul, Nebr., Middle Loup River at ................. 380

North Loup River near .......... 383

Salt Creek at Lincoln, Nebr. . . . . . 394 near Ashland, Nebr. . . . . . . . . 395

Shell Creek at Newman Grove, Nebr. 390 near Columbus, Nebr. . . . . . . 391

Silver Creek at Ithaca, Nebr. . . . . . 396

Silver Creek', Nebr. , Prairie Creek near................. 379

Spalding, Nebr. , Cedar River near .. 385 Spring Creek at Cushing, Nebr. . . . . 384 Stages and discharges at streamgaging stations .......371-372 Summary of flood stages and discharges..........406-407

Talmage, Nebr., damage to railroad track near ........... 357,fig. 54

Thomson Creek at Riverton, Nebr. . 401 Uehling, Nebr., Logan Creek near .. 394 Unadilla, Nebr., view along U. S. Highway $40 \ldots \ldots \ldots$ 357, fig. 53 Union, Nebr., Weeping Water Creek at 397 Wahoo Creek at Ithaca, Nebr....... 396 Waterlooe, Nebr., Elkhorn River at.. 393 Weeping Water Creek at Union, Nebr. 397 Wood River near Gibbon, Nebr. ..... 378 York, Nebr., graph showing rainfall at ............ 362, fig. 58 